\title{
The Forum BEVI Project: Applications and Implications for International, Multicultural, and Transformative Learning
}

\author{
Elizabeth Wandschneider, Michigan State University, lead author \\ Dawn T. Pysarchik, Michigan State University \\ Lee G. Sternberger, James Madison University \\ Wenjuan Ma, Michigan State University \\ Kris Acheson, Georgia State University \\ Brad Baltensperger, Michigan Technological University \\ R.T. Good, Shenandoah University \\ Brian Brubaker, IES Abroad \\ Tamara Baldwin, University of British Columbia \\ Hajime Nishitani, Hiroshima University \\ Felix Wang, James Madison University \\ Jarrod Reisweber, Beck Institute \\ Vesna Hart, James Madison University
}

The only person who is educated is the one who has learned how to learn and change. - Carl Rogers

In a diverse portfolio of curricular and programmatic options in colleges and universities, global education has become highly prominent over the past 50 years (O'Meara, Mehlinger, \& Newman, 2001; De Wit \& Merkx, 2012). To take one of any number of indices in this regard, the Institute of International Education (2014) reported that a record number of global students, 886,052, studied at U.S. institutions in 2013-14 (up 8.1 percent over the previous year) and 289,408 American students studied abroad in 2012-13 (an increase of 2.1 percent over the previous year) (see also International Association of Universities, 2010; Knight, 2006). At a complementary level, institutions of higher education now are interested in "the internationalization of research, offering dual degrees with foreign partners, establishing a branch of campuses abroad," among other areas of emphasis (Marmolejo, 2011). Duru and Poyrazli (2007) contend that these sorts of trends demonstrate the important role that colleges and universities play in training workers for entry into the global marketplace. Likewise, Green (2013) notes that "higher education institutions in the United States are increasingly using the language of 'global citizenship' to describe the skills and habits they seek to cultivate in their students" (p. 52). Against this introductory backdrop, we begin this article with an overview of global education in the United States and a review of assessment instruments and approaches. We then will present 15 implications from the Forum-BEVI Project, a multi-year, multi-site assessment initiative that examined the processes and outcomes of international, multicultural, and transformative learning. ${ }^{1}$

\footnotetext{
${ }^{1}$ With permission from Springer Publishing, this article for Frontiers is adapted from Wandschneider, E., Pysarchik, D.T., Sternberger, L.G., Ma, W., Acheson, K., Baltensperger, B., Good, R.T., Brubaker, B., Baldwin, T., Nishitani, H., Wang, F.,Reisweber, J., Hart, V. (in press). The Forum BEVI Project: Applications and implications for international, multicultural, and transformative learning. In C.N. Shealy (Ed.), Making sense of beliefs and values. New York, NY: Springer Publishing. See http://www.springerpub.com/making-sense-of-beliefs-and-values.html. (C)2015 The Forum on Education Abroad
} 


\section{Global Education in the United States}

In 2005, the Forum on Education Abroad investigated international education programming in 46 U.S. colleges and universities (Forum, 2005). Results suggested that study abroad programs are intimately related to their curricula and objectives. Most of the surveyed institutions integrated global education into the international curricula of their students; 91 percent of the institutions usually or always counted credits earned by students while abroad toward major or minor requirements. Moreover, 77 percent of the respondents noted that credit completed abroad usually or always counted towards general education requirements. Similar to the curricular changes at many U.S. universities, the composition of matriculating students also has changed in the last 50 years. Such students today often have prior international exposure, which is related in part to the fact that transportation to and from other countries is much more accessible. Furthermore, young adults are exposed to the beliefs and practices of different cultures through television, the Internet, and social media. In addition, many students anticipate that cultural competence will be required of them upon graduation from college or university at a level that simply was unimaginable 50 years ago. Thus, it is not surprising that a survey by the American Council on Education (ACE) found that most students studying abroad had prior international experience, with 64 percent of students polled already having lived in or traveled to countries outside of the United States (Siaya \& Hayward, 2003).

Today's students appreciate the importance of grappling with events and issues that are occurring beyond their home borders, in part because of an increasing recognition that global and domestic affairs are inextricably linked. For example, 65 percent of the students in the 2003 ACE survey concurred that all students should be required to take courses covering global topics (Siaya \& Hayward, 2003). Moreover, when entering the global marketplace, graduating U.S. students who have studied abroad arguably possess an advantage over those who have not. Indeed, "students who lack access to cultural capital may not appreciate the significance of study abroad experiences specifically and global understanding in general" (Hu, Pazaki, \& Velander, 2014, p. 73). The advantage of study abroad consists of more than cross-cultural experience, and is related directly to the acquisition of knowledge, skills, and attitudes that cannot be acquired without real world experience in another culture. And in fact, many global education programs are designed explicitly to facilitate the acquisition of greater sensitivity toward people from other cultures (O'Meara, Mehlinger, \& Newman, 2001). As Ramirez (2013) observes, however, such student outcomes are not preordained simply by studying abroad; rather, "the curriculum of these preparation programs needs to intentionally address

cultural sensitivity" (p. 8). Thus, it is the responsibility of institutions of higher education to "develop international curricula that foster a student citizenry with stronger global awareness" (Tarrant, Rubin, \& Stoner, 2013, p. 2).

\section{The Assessment of Global Learning}

As the importance of international education continues to ascend in the U.S. and globally, a corresponding need has emerged to assess the outcomes and impacts of such learning (Deardorff \& Van Gaalen, 2012). In this regard, assessment instruments must be chosen in the context of how a given institution defines internationalization (Coryell, Durodoye, Wright, Pate, \& Nguyen, 2012). Knight (2011) observes further that the benefits of global education cannot be measured simply by counting "the number of global students, foreign faculty, institutional agreements, cross-border education programs, research projects, foreign accreditations, branch campuses, and so on" (p. 15). Such indices may address certain accountability requirements, but they don't reflect the emotional, cognitive, and behavioral impact of globalization. As Zimmermann and Neyer (2013) comment in a study on the personality development of sojourners, "hitting the road has substantial effects on who we are" (p. 527). Although assessment and education, in general, long have been linked in the United States, an emphasis on the assessment of international, multicultural, and transformative learning has been a relatively recent phenomenon, with various attempts underway to define 
and determine appropriate assessment methods of intercultural competencies (e.g., Deardorff, 2006; Fantini, 2009; Rexeisen, 2012). In this regard, Sternberger, Pysarchik, Yun, and Deardorff (2009) contend that an integrated approach to learning assessment - which includes a carefully planned and well-implemented assessment protocol that is linked to theory and uses multiple methods - may be of considerable benefit to higher education institutions, by helping students develop measurable competencies that are critical to success in an increasingly interconnected world. Along these lines, the Georgia Learning Outcomes of Students Studying Abroad Research Initiative (Sutton and Rubin, 2004), the Georgetown Consortium Project (Vande Berg, Connor-Linton, \& Paige, 2009), and the Wabash National Study of Liberal Arts (Salisbury, 2011) are but three large, multi-institutional studies regarding the learning outcomes of education abroad. As summarized in the project Student Experience in the Research University (SERU), "formal study abroad programs ... bring value-added components to students' intercultural and global competencies that generally meet or surpass the outcomes of other international travel opportunities" (Stebleton, Soria, \& Cherney, 2013, p. 15). From the standpoint of assessment, several large-scale initiatives to develop methods and instruments for measuring globalization recently have been pursued in different countries, including, but not limited to, the Association of Commonwealth Universities' Management Benchmarking Program (2012); NAFSA's "Accessing Best Practices in International Education" (2008); the Netherlands' Organization for International Cooperation in Higher Education's "Measuring Internationalization" or MINT project (2012); "A Guide to Outcomes Assessment in Education Abroad" (2007), from the Forum on Education Abroad; and, the ForumBEVI Project (2015) - the focus of this article - which investigates the processes and outcomes of global, intercultural, and transformative learning.

So how has the field of international education approached the evaluation of learning outcomes? Overall, a review of the literature suggests that assessment studies have examined factors such as content knowledge concerning a specific culture or knowledge of global affairs, language acquisition, intercultural sensitivity, competence and communication, and salient personality variables, among other constructs and processes. Recognizing that the relative merits of particular assessment methods depend upon differences in program goals or content as well as realities "on the ground" (Poole \& Davis, 2006), typical assessment methods include tests, portfolios, interviews, reflective essays, surveys, and inventories. Although not intended to be exhaustive, it may be helpful to describe a number of these measures and methods in order to illustrate their different applications in research and practice (e.g., see Fantini, 2009).

Some global learning programs are interested in assessing students' knowledge of issues that reflect global awareness and citizenship. For example, the Global Awareness Profile (GAP) is a self-report inventory that includes 126 questions based on common knowledge in two main categories: seven geographical areas (i.e., Asia, Africa, North America, South America, the Middle East, Europe, and Global) and seven subject areas (i.e., environment, culture, politics, geography, religion, socioeconomics, and global) (Corbitt, 2011).

Language acquisition is another significant component of many global learning programs, which requires its own form of assessment. For example, the American Council on the Teaching of Foreign Languages (ACTFL) developed proficiency guidelines in 2012 to assess writing and speaking proficiency levels as well as oral proficiency, using Oral Proficiency Interviews (OPI) and Simulated Oral Proficiency Interviews (SOPI) (ACTFL, 2012). Likewise, the Interagency Language Roundtable (2011) developed a set of skill level descriptions by which language proficiency tests may be developed and scored.

At the same time, there is increasing recognition that such competence involves more than just proficiency in reading, writing, listening, and speaking. As Scarino (2010) notes, comprehensive evaluation of foreign language learning programs should include cultural aspects of learning, because "in any act of learning, people (as young persons and as learners) are engaged in interpreting self (intraculturality) and other (interculturality) in diverse contexts of social and cultural interaction" (p. 326). Along these lines, the European Language Portfolio (ELP) requires students to assemble three components: 1) a language passport that includes basic information about a student's linguistic identity; 2) a language biography that includes 
language learning goals, progress, and record of language learning and intercultural experiences; and 3) a dossier that contains a selection of a student's work that is representative of his/her foreign language proficiency (Little, 2002).

Although foreign language and global knowledge are included as part of the content of what is learned, much assessment regarding global learning has focused on attitudinal, cognitive, and affective change. As an early example of such measurement over 50 years ago, Sampson and Smith (1957) attempted to measure "world-mindedness," which was concerned with the problems of humanity rather than the problems of a specific nation or culture. The 32-item World-mindedness Scale assesses eight dimensions: religion, immigration, government, economics, patriotism, race, education, and war. In an attempt to update this measure and address various psychometric issues, Hett (1993) developed the Global-mindedness Scale, which reflects attitudes, beliefs, and behaviors, in order to assess "a worldview in which one sees oneself as connected to the world community and feels a sense of responsibility for its members" (p. 143), and consists of five dimensions: responsibility, cultural pluralism, efficacy, global-centrism, and interconnectedness (see Clarke, Flaherty, Wright, \& McMillen, 2009; Kehl \& Morris, 2007). Along related lines, Der-Karabetian (1992) designed the Cross-cultural World-mindedness Scale, which consists of 26 items that evaluate attitudes toward race, religion, immigration, patriotism, economics, war, world government, and global education (DerKarabetian \& Metzer, 1993). Likewise, Shaftel and Shaftel (2010) developed the Intercultural Student Attitude Survey (ISAS), a 35-item scale created to assess changes in the attitudes and future plans of undergraduate students who study abroad. This measure focuses on assessing motivational cultural intelligence, which encompasses "the desire to learn about and function effectively in intercultural situations" (p. 12). More specifically, ISAS measures six constructs: cross-cultural open-mindedness, ability to manage stress, adventurousness, self-confidence, disposition toward a global career, and foreign language study.

A number of related measures focus on intercultural sensitivity, competence, and communication. For example, Chen and Starosta (2000a, 2000b) define intercultural communication competence as an umbrella concept comprised of cognitive (intercultural awareness), behavioral (intercultural adroitness), and affective (intercultural sensitivity) abilities that impact intercultural communication. Bhawuk and Brislin (1992) argue that intercultural effectiveness requires people to be "interested in other cultures ... sensitive enough to notice cultural differences, and ... willing to modify their behavior as an indication of respect for the people of other cultures" (p. 416). Along these lines, Fantini (2000) helpfully sought to organize these levels of analysis by juxtaposing the categories of awareness, attitude, skills, knowledge, and second language proficiency, with four developmental levels of competence under each category: educational traveler, sojourner, professional, and intercultural/multicultural specialist.

Based upon the Developmental Model of Intercultural Sensitivity (DMIS) (Bennett, 1986, 1993; Bennett \& Hammer, 2002), the Intercultural Development Inventory (IDI) is designed to measure one's status and progression through developmental stages, including denial, polarization, minimization, acceptance, and adaptation; thus, the stages of DMIS theoretically represent an individual's increasingly sophisticated capacity to apprehend and accurately experience cultures that are different from their own (e.g., Hammer, Bennett, \& Wiseman, 2003; Rexeisen, Anderson, Lawton, \& Hubbard, 2008). Such stages may be plotted along a continuum representing a person's cultural sensitivity, ranging from monocultural to intercultural mindsets (Hammer, 2012). From the standpoint of DMIS, when people become more interculturally engaged, they are not just becoming more sensitive to a particular culture; rather, their intercultural view and experience will extend to other cultures, including their own.

Regarding processes of navigating different cultures and contexts, the Cross-Cultural Adaptability Inventory (CCAI) assesses an individual's ability to adapt to different cultures. A 50-item self-report measure, the CCAI helps an individual identify his/her strengths and weaknesses within four skill areas of intercultural communication and interaction: emotional resilience, flexibility/openness, perceptual acuity, and personal autonomy (Kelley \& Meyers, 1995; Chang, Yuan, \& Chuang, 2013). The International Education Survey 
(IES) has been used "to assess how an international experience impacts personal and intellectual development" (Rexeisen, Anderson, Lawton, \& Hubbard, 2008, p. 2). Relatedly, Van der Zee and van Oudenhoven (2000) developed the Multicultural Personality Questionnaire, which measures multicultural effectiveness defined as "success in the fields of professional effectiveness, personal adjustment, and intercultural interactions" (p. 293). This measure has five scales: cultural empathy, open-mindedness, emotional stability, social initiative, and flexibility.

As a final example, the Global Perspective Inventory (GPI) and Global Competence Aptitude Assessment (GCAA) measure three dimensions: cognitive (knowing and knowledge: "how do I know?”), intrapersonal (identity and affect: "who am I?"), and interpersonal (social responsibility and social interaction: "how do I relate?") (see Doyle, 2009). Overall, the GCAA measures dimensions of knowledge, skills, and attitudes as well as internal and external readiness to experience different cultures (Braskamp, Braskamp, Merrill, \& Engberg, 2010).

While the above overview is not intended to be exhaustive, it should illustrate the types of measures that are being used within the field of international education assessment as well as the main foci of each approach. To be sure, many additional instruments might have been featured. For example, Fantini (2006) profiles 87 such measures under the aegis of "Assessment Tools of Intercultural Communicative Competence" (see also Fantini, 2009). The breadth of this compendium illustrates both the challenge in selecting the "right" assessment measure for a particular purpose as well as an attendant need to specify standards by which measures should be evaluated, research conducted, and practice implemented within the broader field of international, multicultural, and transformative learning. To provide guidance, the Standards of Good Practice for Education Abroad, developed by the Forum on Education Abroad, offers a comprehensive system by which the goals and practices of assessment may be evaluated. In this regard, "The Forum Standards are intended to be used on an ongoing basis to respond to the practical realities of developing, managing, and assessing education abroad programs" (Whalen, 2011, p. 3). Many of the instruments described above - the IDI and the GPI, among others - as well as the Beliefs, Events, and Values Inventory (BEVI) described below, deliberately attempt to align their approach to measurement with these Forum Standards (e.g., Braskamp, Blair, Shealy, \& Hammer, 2012).

\section{International, Multicultural, and Transformative Assessment: Key Questions}

The above overview of global learning approaches provides a sense of how scholars and practitioners have attempted to measure the impact of international education, broadly defined. In so doing, many questions emerge. For example: "How do we determine which constructs are most and least salient?" "Whose questions are and are not answered?" "Can we actually measure such complex phenomena in a valid and reliable manner?” In addressing such matters, Sternberger, La Brack, and Whalen (2007) observe the following:

Increasingly, faculty and administrators within the field of study abroad are taking a closer and more nuanced look at the experiences of study abroad, considering in more detail the cognitive, emotional, and developmental changes that may occur as a result of the experience (p. 78).

In many ways, the movement to assess multiple dimensions of global learning mirrors what occurred in the U.S. education system 30 years ago, when more sophisticated models and measurement techniques were introduced into U.S. classrooms. Such innovations allowed for the evaluation, monitoring, and improvement of learning over time, while recognizing that students may respond differently to standard instruction in a way that is congruent with their learning and psychological profiles (Lerner, 2003).

Within the field of global education, however, a similar process of understanding and explaining the 
complexities of the interaction of differences among learners with learning processes and outcomes is a relatively recent undertaking. In all likelihood, that is because accounting for learning differences in a traditional environment is compounded substantially by the realities of living and learning abroad. While such learning is similar in some ways to that which occurs in a traditional classroom, it is qualitatively different on another level since study abroad may be experienced and anecdotally referred to as "dramatic" and "life-changing" (Doyle, 2009, p. 143), in part due to the "high impact" nature of such learning (e.g. Iyer, 2013; Kuh, 2008). Thus, students may not experience such learning in the same way or to the same degree. At the same time, substantial outcome variability also is apparent: some students report changes that are minimal, consisting of increased knowledge of facts regarding the foreign culture they visited, or even negative (e.g., "I couldn't wait to get home"); others emerge fundamentally transformed, no longer experiencing self, others, or the larger world as they did prior to the experience (e.g., Cultivating the Globally Sustainable Self, 2015). Given such variability, then, if we truly wish to understand who learns what and why, and under what circumstances, our approach to assessment research and practice must be sophisticated, comprehensive, and sustained in order to account for the complex interactions that we - as educators, scholars, and administrators - sense in our students but don't necessarily directly apprehend (e.g., Church, 1982; Reisweber, 2008; Sternberger, La Brack, \& Whalen, 2007).

Although there is a recent push to measure the affective and cognitive variables that are associated with global learning, assessment research and practice that is designed explicitly to account for such complexity is relatively rare. Instead, much of the scholarship in this area focuses on the acquisition of factual information or attitudinal change in students as well as the mapping of developmental stages such as orientation, study abroad, and re-entry (e.g., Koskinen \& Tossavainen, 2003). Such work is very important and highly compatible with aspects of what is reported below. However, if we are to understand the processes that moderate and mediate change via international, multicultural, and transformative learning, our theoretical models and assessment methods must be able to account for such interacting complexities as they occur for real human beings in real time in the real world. To take but one example, the disequilibrating experience that many students encounter while studying abroad is commonly described as culture shock. As Kohls (2001) observed, culture shock results from:

being cut off from the cultural cues and known patterns with which you are familiar, ... living and/or working over an extended period of time in a situation that is ambiguous; ... having your own values ... brought into question, which yanks your moral rug out from under you; and ... being continually put into a position in which you are expected to function with maximum skill and speed but where the rules have not been adequately explained (pp. 93-94).

Although we concur with the validity of such an observation, the underlying affective, cognitive, cultural, contextual, and developmental processes that "cause" an individual to experience "culture shock" to varying degrees are not sufficiently illuminated by a descriptive level of analysis alone (Reisweber, 2008). In short, despite many helpful and productive advances in this area, the complex variables that mediate and moderate global learning processes and outcomes still await precise identification. Hampering our assessment and scholarly efforts in this regard, as noted by Sternberger et al. (2007), "many of the collection methods still do not follow best practices in research design nor do the researchers collect data in ways that are consistent over time" (p. 79). In the final analysis, then, educators and administrators with an interest in these matters must recognize and account for the profound human complexities that interact with a particular international, multicultural, or transformative learning experience to produce the outcomes that we ultimately observe. To achieve such goals, we must become much more methodologically intentional and sophisticated in our assessment research and practice in order to 1) address the overarching questions of who learns what and why, and under what circumstances and 2) translate our understanding into interventions that demonstrably improve teaching, training, and learning both in and outside of the classroom (e.g., Cultivating the Globally Sustainable Self, 2015; Shealy, in press). 


\section{The Forum BEVI Project ${ }^{2}$}

In an attempt to address the above assessment-based complexities, the Forum on Education Abroad through its Outcomes Assessment Committee partnered with the International Beliefs and Values Institute (IBAVI) in 2007 to design and implement a project using the Beliefs, Events, and Values Inventory (BEVI) in order to assess the processes and outcomes of international, multicultural, and transformative learning. There were many reasons for doing so. In particular, the Forum on Education Abroad has a strong and demonstrable record of supporting research-based activities that have the potential to illuminate relevant data, best practices, and appropriate standards for enhancing quality and impact within the field of international education (see www.forumea.org/). At a complementary level, the mission of the IBAVI is to "explore beliefs and values and how they influence actions, policies, and practices around the world" (see www.ibavi.org/). This partnership therefore was ideally suited to the fundamental goals and purposes of both organizations. Moreover, given the comprehensive nature of the BEVI and its integrated theoretical and applied emphases, this measure was seen as ideally suited to a long-term and large scale research-to-practice agenda. As such, the overarching goal throughout this multi-institution, multi-year initiative was to identify findings, applications, and recommendations that were of the broadest and deepest relevance to the larger field. From the beginning, then, this project was designed deliberately to speak to these larger issues of assessment research and practice vis-à-vis international, multicultural, and transformative teaching, training, and learning including, but not limited to, the BEVI.

Toward such means and ends, the Forum BEVI Project participants committed - from the outset - to the following principles and practices of assessment research and practice (e.g., Braskamp, Blair, Shealy, \& Hammer, 2012; Braskamp \& Engbert, 2014; Deardorff, 2006; Gerber, Gottlieb, Pysarchik, \& Shealy, 2011; Pysarchik, Shealy, \& Whalen, 2007a, 2007b; Shealy, 2014; Sternberger et al., 2007):

1. International, intercultural, and transformative learning is highly complex but measurable.

2. Any credible assessment project requires sufficient understanding of relevant theory, measures, design, data, and analysis by all project members.

3. The differences and similarities between assessment practice (what is done in the field) and assessment research (how we understand what is happening through measurement and analysis) must be reconciled.

4. Appropriate assessment standards and practices must be understood and followed (e.g., ethical conduct, IRB approval).

5. Relevant expertise must be accessed (e.g., research, psychometric, statistical).

6. Sufficient institutional support must be secured (e.g., administrative, financial).

7. Processes of project implementation and execution must be consistent with best practices, and monitored throughout.

A final organizational step involved the creation of the Forum - IBAVI Working Group on BEVI

\footnotetext{
${ }^{2}$ A project of this scope could not have occurred without the deep and abiding support from multiple individuals, institutions, and organizations over many years of work. In particular, we gratefully acknowledge the support and engagement of all who participated in various phases of the Forum BEVI Project as well as the indispensable commitments throughout by the Forum on Education Abroad and International Beliefs and Values Institute. We also are grateful for the statistical work of Wenjuan Ma as well as early contributions from Peter DeMichael and Chad Craun, and for the programming expertise of Craig Salee, assisted early on in the project by Todd Broadbent. We also gratefully acknowledge the editorial assistance of Pamela Roy, former Research and Scholarship Associate, and Joy Milano, Research Intern, in the Office of Study Abroad at Michigan State University, both of whom helped address multiple aspects of manuscript preparation. Although our process was highly fluid and intensive, it is our hope that the applications and implications documented here illustrate what may be learned and accomplished through such sustained and collaborative research of this nature. 
Implementation in Research, Education, and Practice. This working group was charged with: 1) identifying and investigating empirical and theoretical questions that may be pursued via the BEVI, which are relevant to international, intercultural, and transformative research, assessment, and practice, broadly defined; 2) providing guidance regarding how the BEVI may best be used to promote learning, growth, and development in various educational, applied, and practical contexts and settings; and, 3) specifying best practices for usage of the BEVI's individual, group, and organizational report system. ${ }^{3,4}$

\section{Forum BEVI Project Pilot Phase}

As envisioned at the outset, this project would include both pilot ${ }^{5}$ and formal phases of implementation. As such, beginning in 2007 and completed in 2009, the pilot phase involved administration of the BEVI to nearly 2,000 international learning participants in the U.S. and abroad. Statistical analysis narrowed the original number of factors on the BEVI from 40 to 18 , and nearly 60 items were eliminated during the subsequent review process. Reliabilities for most of these scales (i.e., factors) were above 0.80 (no retained scale had a reliability of less than 0.75 ); no factor was retained without an eigenvalue of at least 2; and no item was retained with less than a .30 factor loading. Based upon factor analytic and correlation matrix data, as well as a Schmid-Leiman transformation (essentially a factor analysis of the factor analysis) (Schmid \& Leiman, 1957), the 18 scales of the BEVI long version are organized under a series of headings, which correspond with the basic theoretical framework of this measure (discussed below).

Despite such advances, a number of institutions and organizations still wished for a shorter version of the BEVI for a number of reasons. First, within the context of higher education in particular, assessment demands already were high and student/faculty time was short. Second, although each scale on the long version of the BEVI assessed different constructs, these were interdependent with one another (i.e., by design, and consistent with the interconnected nature of beliefs, the "oblique" nature of factor rotation parameters allowed items to load on more than one factor) (see www.thebevi.com). Information gleaned from separate Exploratory Factor Analyses (EFAs) during its development did much to illuminate how and why specific "beliefs, events, and values" were associated together as they were. However, we long had recognized the need to move beyond EFA in order to determine by Confirmative Factor Analysis (CFA) if and how the EFA structure of the measure held upon administration to a new and separate sample, and to understand the relationship among more parsimonious versions of each scale construct. Thus, from 2011 to 2013, the

${ }^{3}$ We gratefully acknowledge the participation of many faculty, staff, and administrators - and thousands of students in the U.S. and internationally - who participated in various stages of this project. Lead participants and their accompanying institutions included Kris Acheson, Georgia State University; Tamara Baldwin, University of British Columbia; Brad Baltensperger, Michigan Technological University; Cynthia Banks, GlobaLinks Learning Abroad (ret.); Brian Brubaker, IES Abroad; Stephen DePaul, Terry College of Business, University of Georgia; Jennifer Engel, Loyola University Chicago; RT Good, Shenandoah University; Esther Gottlieb, The Ohio State University; Melanie Harding, University of British Columbia; Wenjuan Ma, Michigan State University; Gayle Nelson, Georgia State University; Hajime Nishitani, University of Hiroshima; Gaby Peschiera-Carl, University of South Carolina; Dawn Pysarchik, Michigan State University; Craig Shealy, James Madison University and International Beliefs and Values Institute; Christopher Shirley, Georgetown University; Lee Sternberger, James Madison University; Arnd Wächter, Crossing Borders Education; Christa Walck, Michigan Technological University; Felix Wang, James Madison University; and Elizabeth Wandschneider, Michigan State University.

${ }^{4}$ Selected material in this article (e.g., regarding the BEVI, learning implications) has been adapted from Shealy (2014) and Chapters 2, 3, and 4 in the Making Sense of Beliefs and Values book with Springer Publishing (Shealy, in press). Wenjuan Ma, Forum BEVI Project statistician, is responsible for the initial development of the "short version" psychometrics.

${ }^{5}$ The following colleges, universities, and study abroad program providers, all members of the Forum on Education Abroad, participated in the pilot phase of this project: Brethren Colleges Abroad, Dickinson College, IES Abroad, International Studies Abroad, James Madison University, Michigan State University, Saint Mary's College, Saint Olaf College, University of South Carolina, University of Texas, and Wells College. 
developer of the BEVI (Craig Shealy) coordinated a plan and process with the Working Group to develop a "short version" of the BEVI.

\section{Short Version of the BEVI}

Throughout this process, an overarching goal was to determine if a shorter version of the BEVI, with substantially fewer items, could be developed in a manner that didn't compromise the fundamental integrity of the measure (i.e., continued to illuminate how and why "beliefs, events, and values" were interrelated as they seemed to be via multiple analyses over the years). As any psychometrician will attest, this process was painstaking and intensive. Following a data-scrubbing process (e.g., ensuring that duplicated or incomplete cases were removed from the database), we identified a sample of 2,331 cases to be used in conducting the CFA. This initial phase was more do-able than the next, which required multiple steps to determine which items could be eliminated without sacrificing the integrity of the measure. First, we confirmed which items were loading on which specific scale. Because a number of the long version scales were measuring higher order constructs, it was necessary once again to identify smaller subsets of items (i.e., sub-factors) that comprised the larger construct. We used Cronbach's alpha to select the items that could be removed safely without significantly impacting the consistency of a particular factor (i.e., scale) or its sub-factors (i.e., subscales). We then used analytic methods aligned with Item Response Theory (IRT) to identify the relative contribution of each item to each scale. Again, the overarching goal of this step was to ensure that the short BEVI extracted information about respondents that was similar to the information extracted on the long BEVI. ${ }^{6}$ Although the analyses for this process were relatively straightforward, the challenge lay in the sheer volume of data as well as the need to examine all possible permutations among all items and all scales. Ultimately, we automated these procedures via a "python program," which would stop and output results whenever the Cronbach's alpha coefficient is equal to 0.7 and allow us to compare the respective shapes of the information curve for each version of the BEVI. In other words, to preserve the integrity of the overall BEVI, items loading on the short version needed to evidence a similar capacity to identify the same types of respondents as did the longer version (e.g., regardless of whether someone strongly agreed or strongly disagreed to the items on a particular scale, the short BEVI needed to be able to identify such individuals with equivalent sensitivity to the longer version). The end result of this process was the selection of candidate items for retention and deletion in the development of the short BEVI.

But despite this fundamental step forward, we weren't done yet. That is because the python program often "spat out" different multiple item combinations of various short BEVI scales. To figure out which combination was best for each scale, we used structural equation modeling (SEM) to test all of these possible combinations based upon theoretical propositions that had emerged over years of research with the measure in order to explain "what" specific BEVI scales were assessing and why. This process also was highly iterative, involving a great deal of back-and-forth dialogue between theoretical and statistical perspectives on this measure. Ultimately, we were able to settle on final solutions for all scales that had good statistical and sound theoretical properties. At the conclusion of this process, 40 demographic and background variables (as opposed to 65), 185 items (as opposed to 336), and 17 scales (as opposed to 18) were retained in the short BEVI. ${ }^{7}$ Table 1 summarizes core information regarding these scales.

\footnotetext{
${ }^{6}$ Since most institutions, organizations, and settings are now using the "short version" of the BEVI, and because of the extensive analytic process that resulted in this version, it seems likely as of this writing that the "short version" will become the primary version of the BEVI.

${ }^{7}$ It should be noted that we retained one scale - Identity Diffusion - that did not meet this .70 threshold (it had an alpha of .61). Also, a few items progressing through the first steps did not survive SEM, but were retained nonetheless. On both counts, our reasons for doing so were to identify specific combinations of items for the short version of the BEVI that had the best reliability while also retaining as much fidelity as possible to the longer BEVI. Along these lines, one scale - Global Engagement - appeared to be a sub-factor of a newly named factor, called Meaning Quest, which 
Table 1

BEVI Scale Summaries

\begin{tabular}{lcccc}
\hline & Mean & Std. Deviation & $\begin{array}{c}\text { Cronbach } \\
\text { Alpha }\end{array}$ & $\begin{array}{c}\text { Number of } \\
\text { Items }\end{array}$ \\
\hline Negative Life Events & 2.889 & 0.610 & 0.862 & 9 \\
\hline Needs Closure & 2.646 & 0.290 & 0.712 & 25 \\
\hline Needs Fulfillment & 1.892 & 0.342 & 0.882 & 24 \\
\hline Identity Diffusion & 2.791 & 0.322 & 0.610 & 13 \\
\hline Basic Openness & 2.108 & 0.417 & 0.809 & 12 \\
\hline Basic Determinism & 2.887 & 0.355 & 0.755 & 16 \\
\hline Ecological Resonance & 2.248 & 0.524 & 0.760 & 6 \\
\hline Self Certitude & 2.122 & 0.357 & 0.761 & 13 \\
\hline Religious Traditionalism & 2.705 & 0.782 & 0.903 & 5 \\
\hline Emotional Attunement & 2.175 & 0.421 & 0.814 & 13 \\
\hline Physical Resonance & 2.200 & 0.429 & 0.719 & 7 \\
\hline Self Awareness & 1.855 & 0.358 & 0.810 & 12 \\
\hline Socioemotional Convergence & 1.908 & 0.286 & 0.877 & 36 \\
\hline Sociocultural Openness & 2.058 & 0.287 & 0.798 & 26 \\
\hline Global Resonance & 1.719 & 0.469 & 0.828 & 6 \\
\hline Gender Traditionalism & 2.275 & 0.472 & 0.828 & 11 \\
\hline Meaning Quest & 1.873 & 0.317 & 0.831 & 19 \\
\hline
\end{tabular}

As noted above, to explore scale structure, we used SEM to test the relationships between the items and constructs, a process that was highly iterative. Table 2 summarizes the final model fit information, which indicates that these scales have a relatively good model fit and fit the underlying theory adequately well.

explains why the short version of the BEVI has one less scale than the long version. Extensive review of item combinations also resulted in the renaming of several scales in order to better represent the apparent meaning of each factor. Finally, another round of subject matter expert review of the demographic/background items was conducted as well, which resulted in the elimination of 25 of these items. 
Table 2

Model Fit Information for BEVI Scales

\begin{tabular}{lccccc}
\hline & Chi-square & df & p-value & CFI & RMSEA \\
\hline Negative Life Events & 428.612 & 27 & 0.000 & 0.977 & 0.080 \\
\hline Needs Closure & 2993.316 & 225 & 0.000 & 0.911 & 0.073 \\
\hline Needs Fulfillment & 2855.248 & 248 & 0.000 & 0.912 & 0.067 \\
\hline Identity Diffusion & 28.973 & 2 & 0.000 & 0.983 & 0.076 \\
\hline Basic Openness & 619.225 & 54 & 0.000 & 0.956 & 0.067 \\
\hline Basic Determinism & 536.465 & 41 & 0.000 & 0.927 & 0.072 \\
\hline Ecological Resonance & 456.526 & 9 & 0.000 & 0.967 & 0.147 \\
\hline Self Certitude & 634.634 & 62 & 0.000 & 0.937 & 0.064 \\
\hline Religious Traditionalism & 166.821 & 9 & 0.000 & 0.995 & 0.087 \\
\hline Emotional Attunement & 654.891 & 62 & 0.000 & 0.960 & 0.064 \\
\hline Physical Resonance & 40.557 & 2 & 0.000 & 0.984 & 0.091 \\
\hline Self Awareness & 598.360 & 54 & 0.000 & 0.948 & 0.066 \\
\hline Socioemotional Convergence & 3523.339 & 369 & 0.000 & 0.901 & 0.061 \\
\hline Sociocultural Openness & 2596.628 & 225 & 0.000 & 0.935 & 0.067 \\
\hline Global Resonance & 93.898 & 14 & 0.000 & 0.994 & 0.050 \\
\hline Gender Traditionalism & 765.686 & 44 & 0.000 & 0.948 & 0.084 \\
\hline Meaning Quest & 836.661 & 61 & 0.000 & 0.925 & 0.074 \\
\hline
\end{tabular}

In addition to these key aspects of test development described above, other major activity of the Working Group was to present findings of our work over the six years of this project, which included over 20 publications (e.g., articles, chapters, dissertations), 50 presentations (e.g., symposia, papers, posters), and hundreds of separate analyses. The bulk of this article provides a summary of these applications and implications. Before such an overview, however, it may be helpful to include additional explication regarding the theoretical underpinnings, applications, and scale structure of the BEVI.

\section{Equilintegration Theory and the Equilintegration Self}

Before describing the BEVI in greater detail (e.g., what it actually measures), a brief overview of its theoretical underpinnings - Equilintegration (EI) Theory and the EI Self - is needed. Essentially, Equilintegration (EI) Theory seeks to explain "the processes by which beliefs, values, and 'worldviews' are acquired and maintained, why their alteration is typically resisted, and how and under what circumstances their modification occurs" (Shealy, 2004, p. 1075). Derivative of EI Theory, and informed by scholarship in a range of key areas (e.g., "needs-based" research and theory; developmental psychopathology; social cognition; psychotherapy processes and outcomes; affect regulation; theories and models of "self"), the EI Self seeks to illustrate how the interaction between our core needs (e.g., for attachment, affiliation) and formative variables (e.g., caregiver, culture) results in beliefs and values about self, others, and the world at large that we all internalize over the course of development and across the life span (see Shealy, in press).

\section{The Beliefs, Events, and Values Inventory (BEVI)}

Although we've described various psychometric and development aspects of the BEVI, we haven't yet 
discussed what the BEVI really measures. Concomitant with EI Theory and the EI Self, the BEVI is a comprehensive analytic tool that examines how and why we come to see ourselves, others, and the larger world as we do (i.e., how life experiences, culture, and context affect our beliefs, values, and worldview) as well as the influence of such processes on multiple aspects of human functioning (i.e., learning processes, relationships, personal growth, the pursuit of life goals). The BEVI assesses processes such as: basic openness; the tendency to (or not to) stereotype in particular ways; self- and emotional awareness; preferred strategies for making sense of why "other" people and cultures "do what they do"; global engagement (i.e., receptivity to different cultures, religions, and social practices); and, worldview shift (i.e., to what degree do beliefs and values change as a result of specific experiences). BEVI results are translated into reports at the individual, group, and organizational levels that include a range of scores, indexes, and narratives. Individuals, groups, institutions, and organizations use the BEVI and its report system in a wide range of contexts (e.g., educational, mental health, organizational), and for a variety of applied and research purposes (e.g., to track and examine changes in worldviews over time; to evaluate and improve educational programs or experiences; to cultivate growth and awareness in individuals, couples, and groups; to develop leadership capacity; to enhance organizational functioning; to evaluate whether assessment-based goals are met).

Both the long (336 item) and short (185 item) versions of the BEVI consist of four interrelated components: 1) a comprehensive set of demographic/background items that may be modified for particular projects; 2) a life history questionnaire, which is built into the measure; 3) two validity and 18 (long version) or 17 (short version) scales; and, 4) three qualitative "experiential reflection" items. As a Web-based and Likert-type inventory with four response options (Strongly Agree, Agree, Disagree, Strongly Disagree), the long version of the BEVI typically requires between 35 and 45 minutes to complete; the short version typically requires between 25 and 30 minutes to complete. Items are balanced deliberately to minimize social desirability and other response-set confounds (see Geisinger, 2013; Robinson, Shaver, \& Wrightsman, 1991, 1999). Most importantly, the BEVI is not designed to appraise the debatable correctness or incorrectness of individual responses, but rather to investigate how overall response patterns predict various processes and outcomes, from the beliefs and values associated with ethnocentrism, religious tolerance, partisanship, and gender-based practices and policies to issues of openness, self-access, and emotional attunement.

As noted via multiple chapters in Making Sense of Beliefs and Values - of which this article for Frontiers is included as a chapter - the BEVI has been used for a wide range of purposes (see http://www.springerpub.com/making-sense-of-beliefs-and-values.html). Research-focused chapters explain how the EI model and BEVI method increase our conceptual sophistication and methodological capacity across a range of areas: Culture, Development, Environment, Gender, Politics, and Religion. Practiceoriented chapters demonstrate how the BEVI is used in the real world across a range of applied domains: Assessment, Education, Forensics, Leadership, and Psychotherapy. These implications and applications of the BEVI are illuminated by a number of statistical analyses (e.g., ANOVA [analysis of variance], regression, structural equation models) as well as the BEVI report system. Evidence of validity is indicated by numerous studies demonstrating that the BEVI is able to predict group membership across a wide range of variables including demographics such as gender, ethnic background, parental income, political orientation, and religious orientation as well as other relevant variables and processes as documented in other chapters of this book (e.g., Atwood, Chkhaidze, Shealy, Staton, \& Sternberger, 2014; Brearly, Shealy, Staton, \& Sternberger, 2012; Hill, Johnson, Brinton, Staton, Sternberger, \& Shealy, 2013; Isley, Shealy, Crandall, Sivo, \& Reifsteck, 1999; Hayes, Shealy, Sivo, \& Weinstein, 1999; Patel, Shealy, \& De Michele, 2007; Pysarchik, Shealy, \& Whalen, 2007; Shealy, 2000a, 2000b, 2004, 2005, 2006, 2012; Tabit, Cozen, Dyjak, Pendleton, Shealy, Sternberger, \& Staton, 2011). For example, EI theory, the EI Self, and the BEVI offer an integrative framework and method for predicting and explaining characteristics that are linked to other global issues such as environmental concerns (Patel, 2008) and intercultural awareness (Reisweber, 2008; see also Shealy, Bhuyan, \& Sternberger, 2012). 
Although both "long" and "short" versions of the BEVI are available, many users prefer the short version mainly due to time savings, as described previously. Therefore, the following overview of BEVI scales is based upon the "short version," which overlaps substantially with the "long version." Again, the short BEVI consists of two validity and seventeen process scales. Although there are many ways to present scale scores, they often are presented as a series of colored bars along with a number within each bar. This number corresponds to the percentile score between $1-100$ that an individual or a group scored on that scale based upon their overall response to the items that statistically load on each BEVI scale (i.e., "loading" refers to which items have been shown statistically to cluster together on a specific "construct" or scale of the BEVI, and therefore comprise the items on that scale). The resulting scores are standardized based upon the means and standard deviations for each scale (see Table 1). For group reports, the score is called "aggregate" because it represents the average score for all individuals in the group on a specific scale. BEVI results are presented under nine different domains as indicated below. A description of each of the BEVI scales, under their respective domains, also is provided along with sample items for each scale (in parentheses). ${ }^{8}$ In reviewing the following information, remember that by design, items may load statistically on a given scale in either a negative or positive direction. Thus, if sample items seem to be the opposite of one another, that is both expected and appropriate in terms of the psychometrics of inventories such as the BEVI (i.e., positively and negatively loading items both may comprise a given scale).

\section{Validity Scales}

- Consistency: the degree to which responses are consistent for differently worded items that are assessing similar or identical content (e.g., "People change all the time." "People don't really change.").

- Congruency: the degree to which response patterns correspond to that which would be predicted statistically (e.g., "I have real needs for warmth and affection." "I take my own feelings very seriously.").

\section{Formative Variables}

- Demographic/Background Items: gender, educational level, ethnicity, political/religious orientation, income, etc. (e.g., "What is your gender?" "What is your ethnic background?").

- Negative Life Events (Scale 1): difficult childhood; parents were troubled; life conflict/struggles; many regrets (e.g., "I have had a lot of conflict with one or more members of my family." "My family had a lot of problems with money.").

\section{Fulfillment of Core Needs}

- Needs Closure (Scale 2): unhappy upbringing/life history; conflictual/disturbed family dynamics; stereotypical thinking/odd explanations for why events happen as they do or why things are as they are (e.g., "I had a wonderful childhood." "Some numbers are more lucky than others.").

- Needs Fulfillment (Scale 3): open to experiences, needs, and feelings; deep care/sensitivity for self, others, and the larger world (e.g., "We should spend more money on early education programs for children." "I like to think about who I am.").

- Identity Diffusion (Scale 4): indicates painful crisis of identity; fatalistic regarding negatives of marital/family life; feels "bad" about self and prospects (e.g., "I have gone through a painful identity crisis." "Even though we expect them to be, men are not really built to be faithful in marriage.").

IV. Tolerance of Disequilibrium

- Basic Openness (Scale 5): open and honest about the experience of basic thoughts, feelings, and needs (e.g., "I don't always feel good about who I am." "I have felt lonely in my life.").

- Self Certitude (Scale 6): strong sense of will; impatient with excuses for difficulties; emphasizes positive thinking; disinclined toward deep analysis (e.g., "You can overcome almost any problem if

${ }^{8}$ The BEVI is a copyrighted instrument. BEVI items, item content, scales, or reports may not be modified, copied, disseminated, or published, in whole or part, without the written and express permission of Craig N. Shealy, Ph.D. 
you just try harder." "If you play by the rules, you get along fine.").

\section{Critical Thinking}

- Basic Determinism (Scale 7): prefers simple explanations for differences/behavior; believes people don't change/strong will survive; troubled life history (e.g., "AIDS may well be a sign of God's anger." "It's only natural that the strong will survive.").

- Socioemotional Convergence (Scale 8): open, aware of self/other, larger world; thoughtful, pragmatic, determined; sees world in shades of gray, such as the need for self-reliance while caring for vulnerable others (e.g., "We should do more to help those who are less fortunate." "Too many people don't meet their responsibilities.").

\section{Self Access}

- Physical Resonance (Scale 9): receptive to corporeal needs/feelings; experientially inclined; appreciates the impact of human nature/evolution (e.g., "I am a free spirit." My body is very sensitive to what I feel.").

- Emotional Attunement (Scale 10): emotional, sensitive, social, needy, affiliative; values the expression of affect; close family connections (e.g., "I don't mind displays of emotion." "Weakness can be a virtue.").

- Self Awareness (Scale 11): introspective; accepts complexity of self; cares for human experience/condition; tolerates difficult thoughts/feelings (e.g., "I am always trying to understand myself better." "I have problems that I need to work on.").

- Meaning Quest (Scale 12): searching for meaning; seeks balance in life; resilient/persistent; highly feeling; concerned for less fortunate (e.g., "I think a lot about the meaning of life." "I want to find a better sense of balance in my life.").

\section{Other Access}

- Religious Traditionalism (Scale 13): highly religious; sees self/behavior/events as mediated by God/spiritual forces; one way to the "afterlife" (e.g., "Without religion there can be no peace." "There is one way to heaven.").

- Gender Traditionalism (Scale 14): men and women are built to be a certain way; prefers traditional/simple views of gender and gender roles (e.g., "Women are more emotional than men." "A man's role is to be strong.").

- Sociocultural Openness (Scale 15): progressive/open regarding a wide range of actions, policies, and practices in the areas of culture, economics, education, environment, gender/global relations, politics (e.g., "We should try to understand cultures that are different from our own." "There is too big a gap between the rich and poor in our country.").

VIII. Global Access

- Ecological Resonance (Scale 16): deeply invested in environmental/sustainability issues; concerned about the fate of the earth/natural world (e.g., "I worry about our environment." "We should protect the land no matter who owns it.").

- Global Resonance (Scale 17): invested in learning about/encountering different individuals, groups, languages, cultures; seeks global engagement (e.g., "It is important to be well informed about world events." "I am comfortable around groups of people who are very different from me.").

\section{Experiential Reflection Items}

The BEVI is a "mixed methods" measure in that both quantitative (i.e., scales) and qualitative (i.e., free response) items are asked during administration and used for purposes of interpretation (e.g., Coates, Hanson, Samuel, Ashe, \& Cozen, in press; Cozen, Hanson, Poston, Jones, \& Tabit, in press). The following three qualitative Experiential Reflection Items are included in the BEVI, and completed in written format at the conclusion of administration:

First, please describe which aspect of this experience has had the greatest impact upon you and why? 
Second, is there some aspect of your own "self" or "identity" (e.g., gender, ethnicity, sexual orientation, religious or political background, etc.) that has become especially clear or relevant to you or others as a result of this experience?

Third, what have you learned and how are you different as a result of this experience?

\section{The Forum BEVI Project: Applications}

Throughout the Forum BEVI Project, a variety of research questions, assessment projects, and applied interventions were examined depending upon the emphases and needs of participating institutions and organizations as well as the overarching goals of the project to understand better the processes and outcomes of international, multicultural, and transformative learning. Overall, these activities fell under one or more of the following eight areas of inquiry and practice:

1. evaluating learning experiences (e.g., study abroad, multicultural courses, training programs, service learning, etc.);

2. understanding learning processes (e.g., who learns what and why, and under what circumstances);

3. promoting learning objectives (e.g., increased awareness of self, others, and the larger world);

4. enhancing teaching and program quality (e.g., which experiences, courses, programs have what impact, and why);

5. facilitating growth and development (e.g., of individuals, groups, and organizations);

6. conducting research (e.g., how, why, and under what circumstances people become more "open" to different cultures);

7. addressing organizational needs (e.g., staff/leadership development); and,

8. complying with assessment and accreditation requirements (e.g., linking objectives to outcomes).

In the pursuit of such activities, over 500 different analyses were conducted with various subsets of participants, both within and across participating institutions, which included the generation of thousands of individual reports and dozens of group/organizational reports. Although over 8,000 participants completed the BEVI from 2007 - 2013, many of the results below were developed either at the institutional level or on the basis of a large, cross-institutional dataset $(\mathrm{N}=2331)$ collected toward the conclusion of the project, during 2011 - 2012. ${ }^{9}$ Participants primarily included undergraduate students (96.7 percent) and were recruited through a range of learning experiences (e.g., study abroad, residential learning communities, general education courses with a focus on transformative/multicultural learning). A small portion of graduate students (3.3 percent) also was included. The sample typically ranged between the ages of $17-26$, with an average age of 19. However, 3.9 percent fell into the age range of $26-62$, with another 9 percent falling into the age range of $12-17$. Although the majority of participants reported as U.S. citizens ( 93.3 percent), nonU.S. citizens also were included in the sample $(\mathrm{N}=156$ or 6.7 percent). Also, participants were drawn from 38 different countries of origin. Of the sample, 79.1 percent reported as Caucasian with 20.9 percent as nonCaucasian (6.6 percent Black or African American; .9 percent American Indian or Alaskan Native; 7.4 percent Asian or Pacific Islander; 2.9 percent Hispanic/Latino; 3 percent Other). Finally, from the standpoint of gender, 40.8 percent of the sample was female, with 59.2 percent male. All participants were required to provide informed consent as determined by multiple Institutional Review Boards processes, and participation was entirely voluntary. Participants were not required to complete the BEVI, and could elect to discontinue

\footnotetext{
${ }^{9}$ Note that some of the scale names differ from the short and long versions of the BEVI. In addition to the clarification provided above, please see www.thebevi.com for additional information. 
participation at any time. Analyses were conducted via SPSS and MPLUS, and consisted of ANOVAs, regression analyses, and structural equation modeling (SEM).

\section{The Forum BEVI Project: Implications for International, Multicultural, and Transformative Learning}

Given the scope of this work, it is challenging to select only a small sample of findings from hundreds in order to identify the most salient "lessons learned." Ultimately, it proved most useful to distill all that we discovered through this project into a series of 15 implications for international, multicultural, and transformative learning:

1. Who we are affects whether, what, and how we learn.

2. Education, broadly defined, is associated with - and likely causes - change in beliefs and values.

3. To understand how effective our interventions are, we must assess who learners are.

4. It is possible to predict who will be interested in - and satisfied by - a learning experience even before the learning experience actually occurs.

5. Specific analyses may be conducted for purposes of outreach and engagement.

6. All educators and educational experiences are not equal.

7. Educational experiences are associated with desirable as well as undesirable processes and outcomes across a wide range of constructs that must be measured to be known.

8. Examining only overall (e.g. aggregate) findings or privileging only a few BEVI scales constitute tempting but counter-productive approaches to comprehensive assessment.

9. Although distinct characteristics of various cross-cultural groups are identifiable, broad-based assessment suggests within as well as between group variation.

10. As Kurt Lewin observed, there is nothing so practical as a good theory.

11. It is possible to identify the profile or signature of an institution or organization.

12. Group reports help course instructors, program directors, and administrators understand better the nature of their particular class, cohort, or staff.

13. By jointly utilizing individual and group reports, multiple opportunities for self-assessment, enhanced understanding, and group development may be facilitated.

14. Changes in beliefs and values about self, others, and the world at large appear determined largely by the 7Ds (duration, difference, depth, determine, design, deliver, debrief).

15. Best practice in assessment requires best practice in research.

We discuss each of these implications below, along with relevant findings, applications, and recommendations. ${ }^{10}$

Implication 1. Who we are affects whether, what, and how we learn. Formative variables such as life experiences and demographics interact powerfully with the experiences we provide to learners (e.g., study abroad, multicultural coursework, growth/development workshops, education in general) to influence the learning processes and outcomes that we observe.

${ }^{10}$ The presentation of results in the following section of this article is informed by the March, 2012 Special Section of the journal Psychotherapy, entitled "Ethical Issues in Clinical Writing," Volume 49, Issue 1, pp. 1 - 25 as well as HIPAA regulations, APA ethical guidelines, and other best practices for reporting such information. In this regard, although all assessment and report results are consistent with original patterns and profiles, key information may have been modified on occasion (i.e., specific scores) in order to ensure the anonymity of respondents and/or consistency across the long and short versions of the BEVI.

(C)2015 The Forum on Education Abroad 


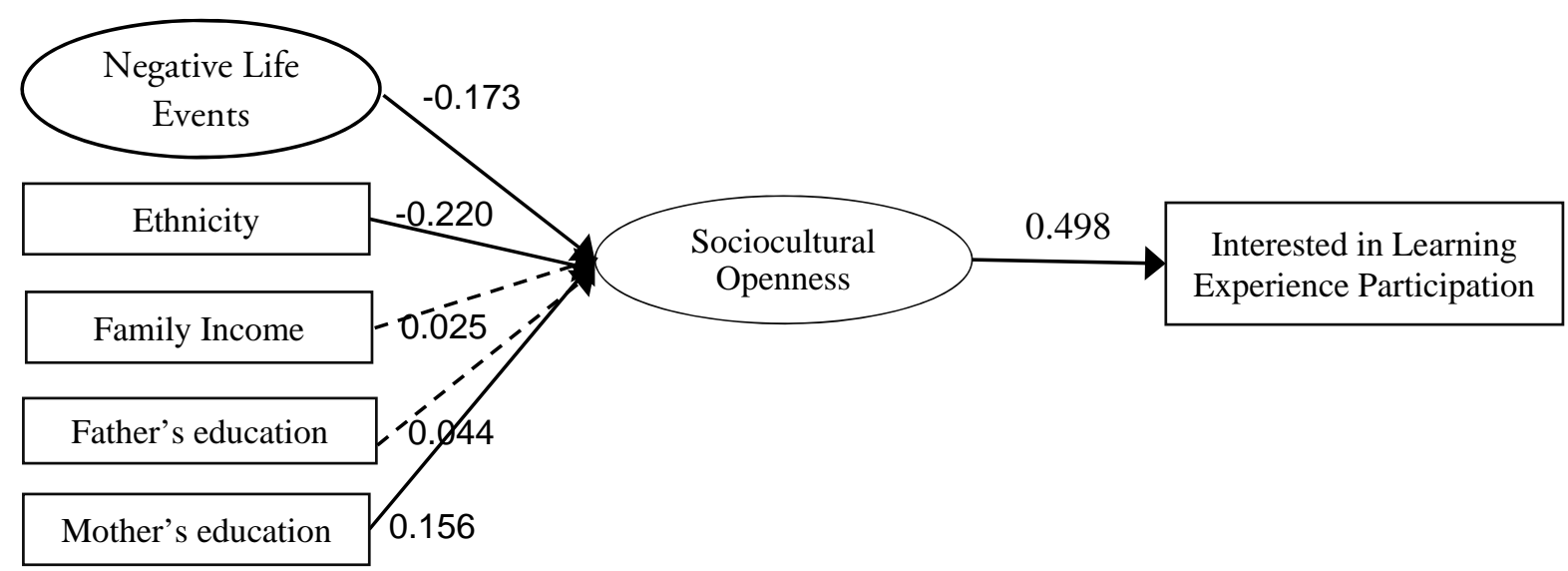

$\mathrm{N}=1016$, Chi-square $=9141.328, \mathrm{df}=1257, \mathrm{p}=0.000, \mathrm{RMSEA}=0.052, \mathrm{CFI}=0.938$

Figure 1. Sociocultural Openness as a mediator between demographic and experiential variables and interest in global learning experiences (international, multicultural, and transformative).

Consider Figure 1, which is a structural equation model (SEM) regarding the relationship between Formative Variables, Mediators, and Outcomes. Essentially, SEM is a sophisticated statistical approach that is derivative of various methods for establishing predictive pathways among constructs that are thought to have a relationship with one another. In other words, SEM allows for theory testing, since this approach evaluates the statistical relationship that is theorized to exist between constructs that have achieved a measure of reliability and validity. Thus the "model" of structural equation modeling essentially is the theory presumed to underlie the relationship among a set of constructs (e.g., Kline, 2010). From the standpoint of the EI model and BEVI method, the SEM pictured in Figure 1 basically contends that formative variables (e.g., antecedent variables that influence why we come to experience self, others, and the larger world as we do) are associated with/predictive of mediating variables (e.g., latent variables that help explain underlying psychological processes that contribute to various outcomes that we observe), which further predict outcomes (e.g., empirically observable outcomes, such as behaviors or verbalizations regarding one's intention or reality).

So, from this perspective - and grounded in the EI theoretical framework - one's stated "interest" in international or multicultural learning is, in no small part, determined by previous life experiences, which shape our "beliefs and values" regarding self, others, and the larger world. This proposed theoretical model regarding the predictive linkage among formative variables, mediators, and outcomes, receives strong support from Figure 1. Here, we used Mplus with means and variance adjusted weighted least squares (WLSMV) as the estimator. Antecedents in the model are: Negative Life Events; ethnicity measured as a dummy variable ( $0=$ minority, $1=$ white/Caucasian); family income as an ordinal measure of the annual family income even if the participant did not receive financial support from them ( $1=$ less than $\$ 10,000 /$ year to $10=\$ 175,000$ + /year); and, father's and mother's education levels (each range from $0=$ some high school or less to $8=$ doctoral degree). The dependent variable is the level of interest in global, multicultural or transformative learning, which is measured by a single question ( $1=$ extremely low interest to $7=$ extremely high interest).

Results show variability in the impact of formative variables on students' interest in international and multicultural learning. Table 3 below provides the parameters of these linkages. Specifically, Negative Life Events and ethnicity are negatively associated with Sociocultural Openness, which means that students 
experiencing fewer Negative Life Events were more Socioculturally Open, and that Caucasian students are less Socioculturally Open than minority students. Moreover, mother's education is positively associated with Sociocultural Openness, while family income and father's education are not significantly related to Sociocultural Openness. Sociocultural Openness also has a positive influence on students' interest in

Table 3

Factors Impacting Interest in Global Learning: Structural Coefficients, SEM

\begin{tabular}{lcc}
\hline & $\begin{array}{c}\text { Unstandardized } \\
\text { coefficient }\end{array}$ & $\begin{array}{c}\text { Standardized } \\
\text { coefficient }\end{array}$ \\
\hline Negative Life Events $\longrightarrow$ Sociocultural Openness & -0.166 & -0.173 \\
\hline Ethnicity $\longrightarrow$ Sociocultural Openness & -0.454 & -0.220 \\
\hline Family Income $\longrightarrow$ Sociocultural Openness & -0.007 & 0.025 \\
\hline Father's Education $\longrightarrow$ Sociocultural Openness & 0.016 & 0.044 \\
\hline Mother's Education $\longrightarrow$ Sociocultural Openness & 0.061 & 0.156 \\
\hline Sociocultural Openness $\longrightarrow$ Global Learning Interest & 1.139 & 0.498 \\
\hline
\end{tabular}

engaging in international, multicultural, and transformative learning experiences. In short, the experience of particular formative variables (positive life events, being non-Caucasian, and having a mother with a higher degree of education) is predictive of the degree to which one is "open" to the beliefs, values, and practices of other cultures, which is further and strongly predictive of one's interest in engaging with and learning from cultures that are different from one's own.

Implication 2. Education, broadly defined, is associated with - and likely causes - change in beliefs and values. Abundant quantitative and qualitative evidence from different types of learning experiences assessed by the Forum BEVI Project (e.g., study abroad, multicultural coursework, general education) indicates that students are likely to see and experience themselves, others, and the larger world differently following such experiences.

Contemplate, first, the degree to which "education" influences the relative sophistication that students demonstrate in terms of their attributions about self, others, and the larger world, a framework that may generally be grouped - from the standpoint of the BEVI - under the domain of "Critical Thinking." What is meant by this construct? Consider a joint statement presented at the 8th Annual International Conference on Critical Thinking and Education Reform (Scriven \& Paul, 1987), in which critical thinking is defined as "the intellectually disciplined process of actively and skillfully conceptualizing, applying, analyzing, synthesizing, and/or evaluating information gathered from, or generated by, observation, experience, reflection, reasoning, or communication, as a guide to belief and action" (see https://www.criticalthinking.org/pages/definingcritical-thinking/766 ). ${ }^{11}$ According to this definition, critical thinking involves a gestalt of competencies that helps an individual assess information (in terms of thoughts, feelings, and behaviors) within oneself and one's surroundings, understand and reason with complexity, and utilize this resulting awareness to understand self, others and the world in more sophisticated and accurate terms.

Along related lines, Cottrell (2005) regards critical thinking as a meta-thinking skill or cognitive process based on intellectual traits, exposure, educational subjectivity, and the disposition to use such capacities. Another comprehensive definition of critical thinking that emphasizes the "self" is offered by Paul and Elder (2006), which contends:

Critical thinking is, in short, self-directed, self-disciplined, self-monitored, and self-corrective thinking. It presupposes assent to rigorous standards of excellence and mindful command of their use. It entails effective communication and problem solving abilities and a commitment to

${ }^{11}$ Aspects of this section are excerpted and/or adapted from Iyer (2013). 
overcome our native egocentrism and sociocentrism (p. 4).

Finally, highlighting the interrelated complexity of this construct, Manaloa, Kusumib, Koyasub, Michitac, and Waseda (2013) observe that scholars have defined the construct of "critical thinking" in any number of ways, to include:

- "skilled and active interpretation and evaluation of observations and communications, information and argumentation," (Fisher \& Scriven, 1997, p. 21)

- “correct assessment of statements," (Ennis, 1962, p. 81)

- "identification and evaluation of implicit theoretical assumptions," (Yanchar, Slife, \& Warne, 2008, p. 265)

- "an attitude or disposition to recognize when a skill is needed and the willingness to apply it" (Halpern, 1998, p. 452).

The importance of critical thinking, and the relative effectiveness of higher education in facilitating its development and expression, was brought home dramatically by Academically Adrift (Arum \& Roksa, 2011), which concluded that student advances in "critical thinking" were modest at best, mainly because of a lack of rigor (e.g., limited reading and studying requirements). We appreciate this important contribution to a significant question regarding the efficacy of higher education, noting that such issues often come down to matters of measurement (e.g., from a quantitative standpoint, which items load statistically on a specific scale) and operationalization (e.g., the steps or operations that are assembled to define and demonstrate the construct in question, including "critical thinking").

In this regard, from the standpoint of the BEVI and its underlying EI theoretical framework, the construct of "critical thinking" is resonant with several of the above perspectives (e.g., Manaloa et al., 2013; Scriven \& Paul, 1987). Specifically, "critical thinking" is evidenced mainly by the degree of attributional sophistication regarding why people do what they do as well as why various events or phenomena occur as they do, and under what circumstances (Shealy, 2004; Shealy, in press). From a construct level of analysis, on the long version of the BEVI, such processes are operationalized via four scales: Causal Closure, Basic Determinism, Divergent Determinism, and Socioreligious Closure. Among other applications, through the report system described below, the BEVI assists students, instructors, and program directors alike in reflecting on these aspects of critical thinking in order to assess and practice greater self-reflexivity toward self, others, and the larger world (e.g., Ethics of International Engagement and Service-Learning Project, 2011; Iyer, 2013). From the standpoint of the Forum BEVI Project, we wished to examine whether the relatively straightforward moderator of "number of years in college" was associated with critical thinking as defined by the BEVI.

Structural equation modeling (SEM) again was chosen to analyze the relationship between the number of years in college and dimensions of critical thinking. As Figure 2 illustrates, three of the four BEVI scales that are intended to measure "critical thinking" are in fact statistically associated with years in college. What do such results suggest? Essentially, individuals who score highly on Basic Determinism tend to prefer basic/simple explanations for why people are as they are or do what they do (e.g., are inclined towards simple explanations of complex, biopsychosocially-mediated phenomena such as ethnic or gender-based differences among people). Individuals who score highly on Divergent Determinism tend to eschew commonly accepted answers or understandings of phenomena, prefer non-convergent explanations and non-traditional policy solutions, are unconventional, tend to question authority, may reflexively be contrarian (i.e., for the sake of being so), and resist being pinned down (e.g., will tend to contest the validity or truth of whatever claims are being made). Individuals who score highly on Socioreligious Closure tend to have strong religious beliefs and great faith, believe that God alone provides happiness/health, and perceive little personal control. In short and mindful of the possibility for correlation/causation confusion - the more years in college, the higher the 
overall degree of critical thinking, as evidenced by lower scores on Basic Determinism, Divergent Determinism, and Socioreligious Closure.

\begin{tabular}{|c|}
\hline Years in College \\
Key Findings: \\
A higher number of \\
years impacts \\
critical thinking as \\
defined by the \\
BEVI.
\end{tabular}

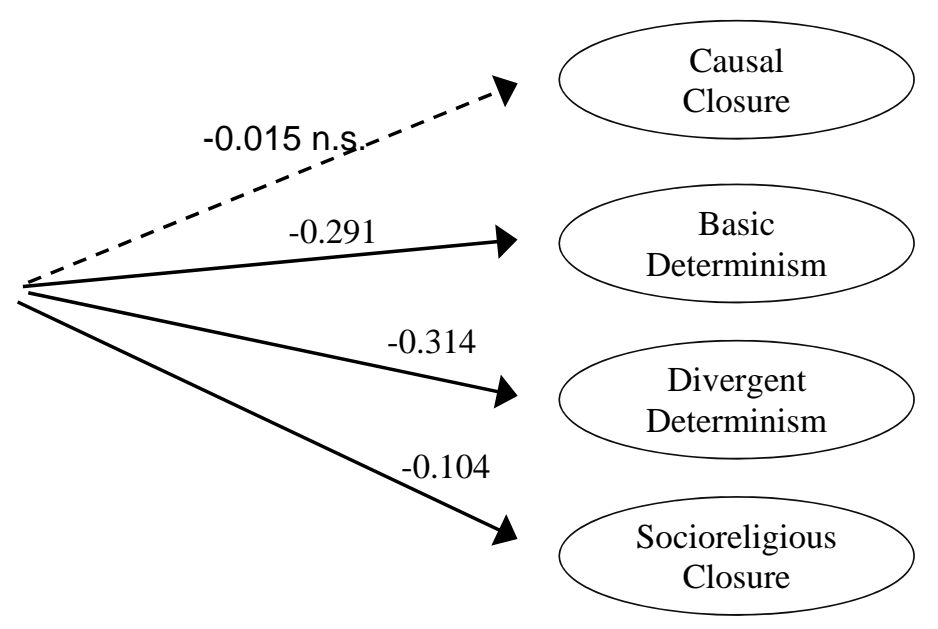


Chi-square $=1579.701 \mathrm{df}=149, \mathrm{p}=0.000, \mathrm{RMSEA}=0.094, \mathrm{CFI}=0.912$

Figure 2. The relationship of "critical thinking" scales on the BEVI to number of years spent in higher education.

Consider a more fine-grained analysis by examining correlation matrix findings regarding Divergent Determinism, the scale that is most strongly negatively associated with number of years of college (i.e., lower Divergent Determinism is associated with more years of college). A primary usage of correlation matrices is to understand the underlying relational patterns among constructs (i.e., scales) which may relate to one another. In so doing, the deliberately oblique factor structure of BEVI scales should be recognized. In other words, as is predicted by the EI theoretical model, and in keeping with the "web-based" nature of "beliefs and values," from a factor rotation standpoint, items are permitted to load on one or more scales, which in fact is key to "making sense" of why beliefs and values are related to each other, and to life events, in the way that they are (i.e., as they naturally occur for real human beings) (Shealy, in press). In this regard, we examine in Table 4 the strongest correlations between Divergent Determinism and other BEVI scales (those at .40 or above).

Table 4

Correlation Matrix Findings above .40 between Divergent Determinism and other BEVI Scales

\begin{tabular}{lr}
\hline Scales & Correlation \\
\hline Socioemotional Convergence & -0.81 \\
Needs Closure & 0.65 \\
Emotional Attunement & -0.58 \\
Negative Life Events & 0.53 \\
Sociocultural Openness & -0.50 \\
Basic Closedness & 0.45 \\
Ecological Resonance & -0.43 \\
Identity Diffusion & 0.42 \\
\hline
\end{tabular}


What do such findings suggest? As Table 4 illustrates, correlation matrix data suggest individuals who score highly on Divergent Determinism also:

- are likely to have greater difficulty "holding complexity," tending to see the world in "black and white" terms (Socioemotional Convergence);

- are more likely to indicate that core needs (e.g., for attachment, affection) were not met in a "good enough" manner (Needs Closure);

- are less likely to indicate the capacity and inclination to experience affect in self and other, and to value its expression (Emotional Attunement);

- are more likely to report the experience of a high degree of unhappy life experiences, during childhood/adolescence or generally in life (Negative Life Events);

- are less likely to be interested in or open to the beliefs, values, and practices of cultures that are different from one's own (Sociocultural Openness);

- are more likely to deny or ignore fundamental thoughts, feelings, or needs that generally are experienced as "normative" regarding human existence or functioning (Basic Closedness);

- are less likely to be concerned about or invested in matters that have to do with the environment and natural world (Ecological Resonance); and,

- are more likely to express feelings of confusion and entrapment regarding their current existence and future prospects (Identity Diffusion).

Taken together, these SEM (Figure 2) and correlation matrix findings (Table 4) suggest that critical thinking as defined by the BEVI should be understood not only in terms of the relative attributional sophistication of human beings (e.g., the ability to understand the complexities of why we think, feel, and do what we do or why events happen as they do in the larger world). At a more complex level, it also appears that such capacities and inclinations may in fact be associated with our life histories, since a higher degree of Negative Life Events and Needs Closure appears to be associated with a lower degree of critical thinking as defined by the BEVI, an outcome that would in fact be predicted by the basic needs-based postulates of the EI model (Shealy, in press; Shealy et al., 2012). In any case, the most basic finding here is that the relative sophistication and complexity of attributional tendencies about why people do what they do, or why events happen in the world as they do, increases with the more years of college or university that people experience. Although subject to further inquiry, and ever mindful of the possibility for correlation/causation confusion (e.g., the role of maturational effects), such results suggest that repeated exposure to complex concepts combined with multiple experiences in which one is required to understand, reflect upon, and justify the rationale for beliefs and values in self, others, and the larger world may in fact be associated with the acquisition of more sophisticated attributional frameworks over time.

Implication 3. To understand how effective our interventions are, we must assess who learners are. Attributing positive ratings of learning experiences only or largely to the experience itself, without accounting for who students are prior to their engagement in the experience, is neither good practice nor empirically indicated.

To what degree does exposure to international, multicultural, and transformative learning actually change fundamental aspects of an individual's personality or identity, indeed the very "self" of the learner? Put in other terms, does exposure to such learning experiences cultivate the capacity to care in a deeper way for self, others, and the larger world (Shealy et al., 2012)? Such questions are complicated in their own right, and rendered more so by the fact that individuals who self-select to engage in such experiences may already be "in the choir to which we sing" (i.e., they "get" what we are seeking to facilitate even before the learning experience begins). Multiple findings from the Forum BEVI Project suggest that "who we are" prior to the experience in which we engage may interact profoundly with the experience itself to influence outcomes. Consider the following correlation matrix data from one of the "primary" factors on the BEVI, Sociocultural 
Openness.

Table 5

Correlation Matrix Findings above .40 between Sociocultural Openness and other BEVI Scales

\begin{tabular}{lc}
\hline Scales & Correlation \\
\hline Needs Closure & -0.90 \\
Ecological Resonance & 0.88 \\
Socioemotional Convergence & 0.82 \\
Basic Closedness & -0.81 \\
Identity Diffusion & -0.71 \\
Emotional Attunement & 0.77 \\
Socioreligious Traditionalism & -0.62 \\
Hard Structure & -0.58 \\
Negative Life Events & -0.57 \\
Divergent Determinism & -0.50 \\
\hline
\end{tabular}

What do such findings suggest? Essentially, individuals scoring high on Sociocultural Openness tend to endorse a worldview that may be characterized as liberal, progressive, accepting, culturally attuned, open, concerned, and globally oriented. As Table 5 illustrates, individuals who score highly on Sociocultural Openness:

- are more likely to indicate that core needs (e.g., for attachment, affection) were met in a "good enough" manner (Needs Closure);

- are more likely to be concerned about or invested in matters that have to do with the environment and natural world (Ecological Resonance);

- appear more able to experience the world in complex "shades of gray," rather than in black and white terms (Socioemotional Convergence);

- are less likely to deny or ignore fundamental thoughts, feelings, or needs that generally are experienced as "normative" regarding human existence or functioning (Basic Closedness);

- are less likely to express feelings of confusion and entrapment regarding their current existence and future prospects (Identity Diffusion);

- are more likely to indicate the capacity and inclination to experience affect in self and other, and to value its expression (Emotional Attunement);

- are less likely to endorse a traditional worldview regarding the nature and purpose of religion and its centrality to one's own life (Socioreligious Traditionalism);

- are less likely to express very strong certitude regarding the correctness of one's own way of seeing self, others, and the larger world, while denying doubts or weakness (Hard Structure);

- are less likely to report the experience of a high degree of unhappy life experiences during childhood/adolescence or generally in life (Negative Life Events); and,

- are less likely to adopt a reflexively contrarian posture regarding the nature of "truth" or "reality" (Divergent Determinism).

In many ways, a high degree of Sociocultural Openness may be seen as "optimal" in terms of the goals that were articulated by various institutions and organizations with which we worked throughout the Forum BEVI Project. Again, however, it should be emphasized that a high - or low - degree of Sociocultural Openness may serve as cause and/or effect. For example, individuals high in Sociocultural Openness may be 
predisposed toward study abroad or service learning even before the experience occurs, which therefore may function as more of a mediating effect on observed outcomes than whatever experience they encounter. Thus, it is very important to assess such attributes before and after a learning experience in order to be able to "tease out" the degree to which the experience itself - or who someone already was before the experience - is responsible for whatever changes we observe. In any case, multiple findings suggest that the construct (i.e., scale) of Sociocultural Openness is in fact associated with the sorts of outcomes that appear to be at the core of most international, multicultural, and transformative learning interventions (e.g., study abroad, courses, workshops), in that a higher degree of Sociocultural Openness is predictive of a range of real world outcomes, such as those described next.

Implication 4. It is possible to predict who will be interested in - and satisfied by - a learning experience even before the learning experience actually occurs. If formative variables (e.g., life history, demographics) and mediators (e.g., affective capacity, attributional styles) are assessed with sufficient breadth and depth, it is possible to predict statistically who is, and is not, likely to be inclined toward participation in a learning experience - and who is, and is not, likely to report that they actually benefitted from andlor were transformed by a learning experience - even before the experience occurs.

Such information matters to institutions and organizations for many reasons, including: 1) understanding the "profile" of who was and was not inclined to engage in such experiences; 2) providing outreach to students who historically were not inclined to participate in such experiences in order to try and facilitate their engagement, and; 3) identifying which experiences were more or less likely to elicit interest and/or satisfaction. Overall, multiple analyses conducted through different methodologies indicate that we were able to ascertain various profiles - both from background information and BEVI scales - regarding who was and was not likely to be interested in participating in and/or satisfied by such experiences, even before such individuals completed their first course. Consider, for example, Table 6, which identifies a set of variables from the BEVI that predict the degree to which respondents will report an interest in specific international or multicultural learning experiences. ${ }^{12}$

${ }^{12}$ The dependent variable is a student's level of interest in international education or study abroad. The question is as follows: "On a scale of 1-7, with 1 being 'extremely low' and 7 being 'extremely high,' please indicate your level of personal interest in international education or study abroad experiences." The independent variables are demographic and experiential variables. Several of the independent variables are dummy variables: gender $(0=$ male, $1=$ female $)$; "parents paying for international experience" ( 0 = parents do not pay, 1 = parents pay); "university provides orientation for international experience" ( $0=$ university does not provide, $1=$ university provides); "plan to travel abroad" $(0=$ no plan to travel abroad, 1 = plan to travel abroad); "plan to take an internationally focused course" $(0=$ no plan to take a course, 1 = plan to take a course); and, "speak a foreign language other than English" ( $0=$ does not speak a foreign language, 1 = speaks a foreign language). Another independent variable, the "highest academic degree intended to achieve" is coded from 1 = associate degree, 2 = bachelor's, 3 = master's, 4 = specialist (e.g., Ed.S), $5=$ professional (e.g., law), 6 = doctoral degree. Also, participants are asked about the number of foreign countries they have previously visited (e.g., respondents indicate the actual number of countries they have visited). Background variables include "mother's education" and "family income." "Mother's education" indicates the highest academic degree of a respondent's mother, which ranges from $0=$ some high school or less to $8=$ doctoral degree. "Family income" is an ordinal variable that reflects the average annual income of a student's parents/guardians regardless if the student receives financial support from them. Income ranges from 1 to $10,1=<\$ 10,000$ to $10=>\$ 175,000$. 
Table 6

Predictors of Interest in Global Learning Experiences ${ }^{1}$ : Multiple Regression Analysis

\begin{tabular}{|c|c|c|c|c|c|}
\hline & $\begin{array}{l}\text { Unstandardized } \\
\text { Coefficients }\end{array}$ & & $\begin{array}{l}\text { Standardized } \\
\text { Coefficients }\end{array}$ & & \\
\hline & & Std. Error & Beta & $\mathrm{t}$ & Sig \\
\hline (Constant) & 2.864 & 0.173 & & 16.585 & 0.000 \\
\hline Gender $(0=\mathrm{M}, 1=\mathrm{F})$ & 0.862 & 0.094 & 0.240 & 9.194 & 0.000 \\
\hline Parents are paying for int'l experience & & & & & \\
\hline $\begin{array}{l}(0=\text { Parents do not pay, } 1=\text { Parents pay }) \\
\text { University provides orientation for international } \\
\text { experience }\end{array}$ & 0.915 & 0.106 & 0.238 & 8.599 & 0.000 \\
\hline ( $0=$ No orientation, $1=$ Orientation) & 0.430 & 0.105 & 0.110 & 4.110 & 0.000 \\
\hline Family income & & & & & \\
\hline$(1=<\$ 10,000$ to $10=>\$ 175,000)$ & -0.073 & 0.019 & -0.103 & -3.841 & 0.000 \\
\hline Plan to travel abroad & & & & & \\
\hline $\begin{array}{l}\quad(0=\text { No plan, } 1=\text { Has plan }) \\
\text { Hiahest academic dearee intend to obtain }\end{array}$ & 0.598 & 0.092 & 0.165 & 6.504 & 0.000 \\
\hline ( $1=$ Assoc. degree to $6=$ doctoral degree $)$ & 0.114 & 0.030 & 0.094 & 3.780 & 0.000 \\
\hline $\begin{array}{l}\text { Plan to take an int'l focused course } \\
\quad(0=\text { No plan, } 1=\text { Has plan })\end{array}$ & 0.659 & 0.102 & 0.167 & 6.492 & 0.000 \\
\hline $\begin{array}{l}\text { Number of foreign countries visited } \\
\text { (actual number) }\end{array}$ & 0.031 & 0.014 & 0.056 & 2.178 & 0.030 \\
\hline $\begin{array}{l}\text { Speak a foreign language other than English } \\
\text { (0=Does not speak, } 1=\text { Speaks }) \\
\text { Mother's education }\end{array}$ & 0.336 & 0.088 & 0.095 & 3.813 & 0.000 \\
\hline$F \quad(0=$ Some high school to $8=$ doctoral degree $)$ & $\begin{array}{l}0.074 \\
71.319^{\star \star \star}\end{array}$ & 0.025 & 0.080 & 3.030 & 0.003 \\
\hline $\mathrm{R}^{2}$ & 0.415 & & & & \\
\hline Adj. $R^{2}$ & 0.409 & & & & \\
\hline
\end{tabular}

${ }^{1}$ Global learning experiences $=$ international, multicultural, and transformative learning experiences. $\star \star \star ~(p<.05)$

Multiple regression analysis revealed that among the demographic and experiential background factors, gender and parents paying for international experiences prove to be the best estimators of students' global interests, based upon the respective standardized coefficients. Specifically, female students are more interested in global learning experiences than males. Intuitively logical, those students whose parents paid for their international experiences also have a greater interest in global experiences. Similarly, students who plan to travel abroad, plan to take an internationally focused course, and speak a foreign language have a greater interest in global learning experiences than those who do not. "Highest academic degree intend to obtain" and "Number of foreign countries visited" also are positively associated with interest in global learning experiences. "Mother's education" is a relatively weak but positive estimator. Finally, "Family income" holds a negative association with a student's interest in global learning, meaning the higher one's income, the lower one's interest in global learning. The results of this study suggest that a range of BEVI variables can statistically predict interest in global learning, even before such learning occurs (i.e., at the planning stage), a finding with implications for recruitment, orientation, program development, and institutional planning (e.g., if our goal is to "reach" those students who are least likely to study abroad, such data offer perspective regarding who such students are most likely and least likely to be, although specific variability within a given institution or organization obviously should be considered).

In many ways, "interest" in international, multicultural, and transformative learning experiences represents one bookend of a process that is of great relevance not only to the research community, but to program directors, course instructors, and administrators who want to understand whether specific cohorts of students are inclined toward, or against, various learning experiences. But the other bookend of considerable relevance concerns whether students actually are satisfied by the experiences in which they engaged, and 
whether the BEVI is able to predict such satisfaction even before the learning experience occurs. As Table 7 illustrates, a range of formative variables are statistically associated with such satisfaction. Here, the dependent variable is a student's level of satisfaction across multiple international, multicultural, or transformative learning experiences. Specifically, on a scale of 1-7 (with 1 being "extremely low" and 7 being "extremely high"), the question is as follows: "If you have participated, or are currently participating, in international, learning, service, study abroad, travel, or work experiences ${ }^{13}$ please indicate your overall level of satisfaction." The independent variables are demographic and experiential variables.

${ }^{13}$ For clarification, several of the variables in Table 7 are not part of the "satisfaction" question on the BEVI, but still emerged as relevant for this analysis, albeit moderately (year in university) or weakly (family income, Democrat political orientation, gender, and whether parents traveled abroad). "Satisfaction" options on the BEVI regarding international, multicultural, or transformative learning include the following: 1) completing an internship with a nonU.S. company (in the United States or abroad); 2) living with a roommate from another country or different ethnic background; 3) participation in a course with an international / multicultural focus; 4) participation in on-campus international/multicultural events; 5) service learning in the United States; 6) service learning abroad; 7) study abroad; 8) studying a language other than English; 9) travel to five or more states in the United States; 10) travel abroad; 11) work abroad; 12) living in a residence that is international/multicultural and/or language-focused; 13) participating in offcampus international or multicultural events; 14) have not previously participated in any of these experiences; and 15) other global or international/multicultural experience(s) (please specify).

(O2015 The Forum on Education Abroad 
Table 7

Satisfaction with International Education / Study Abroad Based upon Demographic Variables (Strongest, Moderate, and No Association)

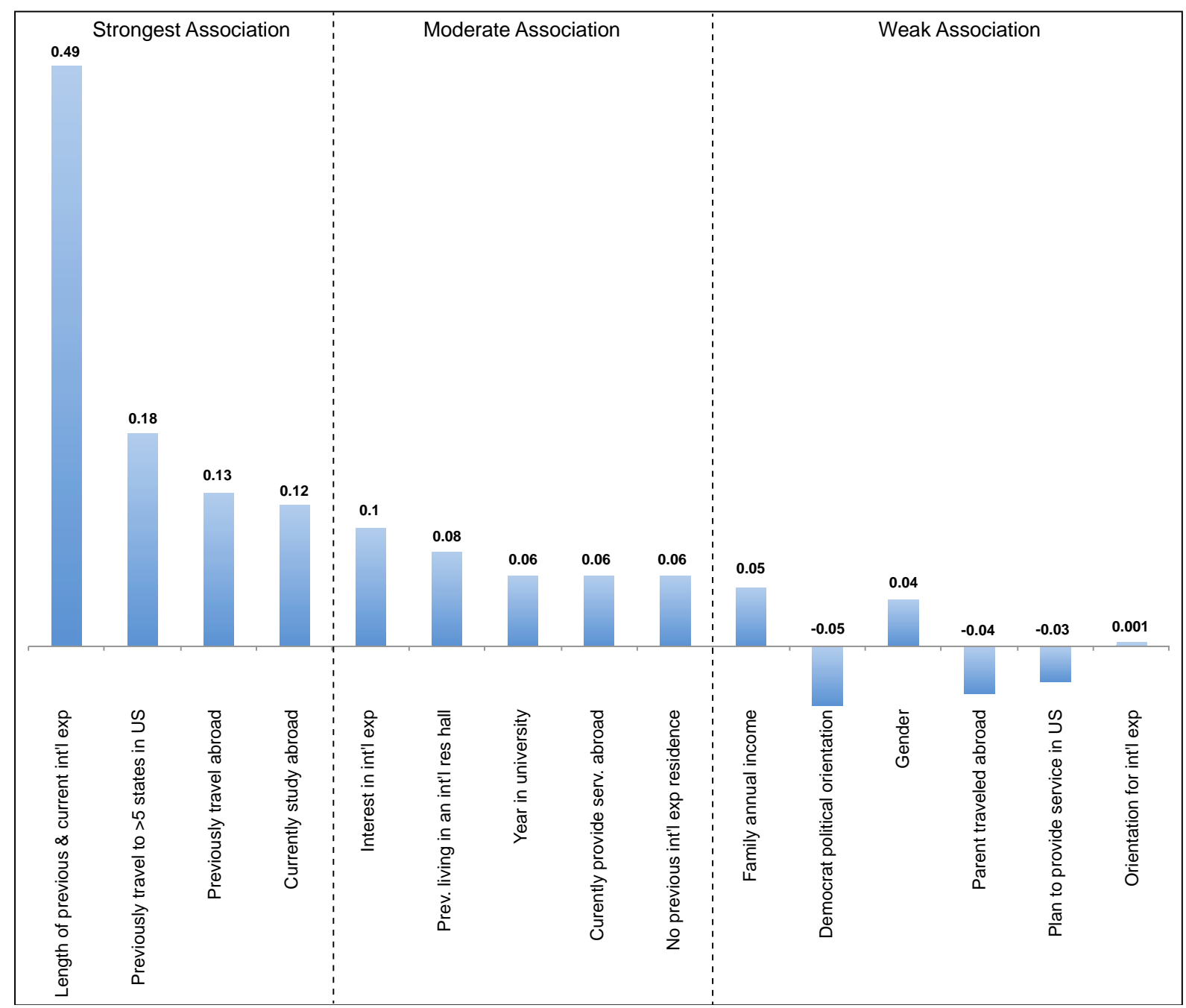


Multiple regression analysis revealed that among the demographic and experiential background factors, the following were most strongly associated with participant satisfaction: a longer length of time participating in one or more of the experiences; previous travel to five or more states in the U.S.; previous travel abroad; and, currently studying abroad. In addition, the above noted variable of "interest" in international experiences - previously living in an international residence hall; and, a greater number of years in higher education - all were among the variables associated with a "moderate" degree of "satisfaction." In short, the results of this study suggest that a range of formative variables statistically can predict satisfaction in international, multicultural, or transformative learning - broadly defined - even before such learning occurs. ${ }^{14}$ Although the ability to predict "interest" is one matter, the fact that we also can predict satisfaction on the basis of formative variables may seem surprising. However, such findings are highly congruent with an EI perspective, in that abundant evidence indicates that the kinds of life experiences we do and do not have are associated inevitably with how we experience self, others, and the world at large, including but not limited to, the satisfaction we ultimately report from engaging in a range of learning experiences.

Implication 5. Specific analyses may be conducted for purposes of outreach and engagement. Because we are able to identify subgroups of students who are more or less likely to be inclined toward specific learning experiences, it is possible to offer targeted opportunities or programs for them that are congruent with institutional goals (e.g., to boost study abroad by minority students). Knowing what sorts of learning experiences are most impactful for which sorts of students also helps clarify and refine interventions that are designed to retain and engage students.

One pressing question both within the field of international education - and in higher education generally as well as other performance-based organizational contexts - is whether and how assessment results actually matter in the pursuit of various institutional/organizational goals and objectives. From promoting individual learning at the student or staff level, to enhancing the quality of courses or programs, to evaluating the degree to which institutions or organizations meet their objectives, these "rubber meets the road" issues are not only reasonable, but inextricably linked to the demonstration that what we do as educators and "change agents" actually matters in the real world (e.g., Blair, Latz, Sternberger, \& Shealy, 2014; Braskamp \& Engberg, 2014). Ultimately, of course, administrators are, or should be, concerned with the justifications for our actions or policies. Such considerations concern matters of learning impact, of course, but also should have implications for matters of quality assurance and cost. For example, presuming the validity of our assessment models and methods, and following best-practice efforts to enhance their quality (e.g., Geisinger, 2013; Robinson, Shaver, \& Wrightsman, 1991), it does not seem unreasonable to use data for the purpose of evaluating programs and courses, and indeed, those who direct or teach them. Throughout this project, we have seen enormous variance in the quality of such administration and teaching vis-à-vis program and course outcomes (see Implication 6 below). Of course, the key is translating the complexity of assessment data into a form that fairly and appropriately may be used to make pragmatic decisions. Given the importance of "effective educational practices" identified by the Association of American Colleges and Universities (AAC\&U, 2007), there can be no substitute for valid assessment of such "high impact" offerings to determine how they may be improved and whether they are achieving their potential (see Iyer, 2013; Kuh, 2008).

For example, consider the below finding. In this case, Shenandoah University wanted to ascertain whether there are differences between students who did and those who did not apply for a short-term learning abroad program $(\mathrm{N}=57)$. Such information was of relevance because this institution was interested in

${ }^{14}$ As indicated by other findings in this article, it should be noted that in addition to these formative variables on the BEVI, multiple scales also predict "interest" in and "satisfaction" with international, multicultural, and transformative learning experiences. For example, in one analysis, Sociocultural Openness, Needs Fulfilllment, Socioemotional Convergence, Global Engagement, Ecological Resonance, Causal Closure, Basic Determinism, Self Awareness, and Gender Traditionalism all were strongly or moderately predictive of "interest" in such experiences (e.g., Shealy, 2014; in press). 
knowing why a program that was deliberately designed to be accessible to students (e.g., provided by the institution at no cost to the student participant) - while also serving as a gateway to other international experiences - was not completed by everyone. From the standpoint of the BEVI, one hypothesis is that students who applied were more psychologically predisposed to do so. We used a binary variable (students did or did not apply to participate) to group the participants into two categories and compared the differences between them. As a dependent variable, we ran a Logit Model to detect differences between the two groups. What did we find? Compared to non-applicants, program applicants were more open to and interested in the attributes and practices of cultures that were different from their own (Sociocultural Openness, $b=7.473, p$ $=0.039$ ). Results such as these have implications for outreach and engagement with different types of students. That is because it is possible not only to know at the outset of study (i.e., during the first year) which students are most and least likely to take advantage of the learning experiences offered to them (i.e., "the who"), but also to gain a measure of understanding as to the underlying dispositions that mediate for or against engagement (i.e., "the why"). In terms of "best practices" for approaching students who are less inclined to engage in these types of learning experiences, it is important to appreciate that these psychological processes help us to differentiate among students while also offering guidance regarding the kinds of interventions that are more likely to "reach" them. Again, in many cases, we sing to the choir, not recognizing that the students who enroll in various learning experiences are self-selecting to do so. More challenging is reaching out to those students who may not be predisposed initially to such experiences - that is, they are low on Sociocultural Openness - but might be willing to move in that direction if we respond to them in an informed, sensitive, and sophisticated manner that better accounts for who they are at the outset (i.e., thus approaching them where they are rather than where we wish them to be) (e.g., Tabit, Legault, Ma, \& Wan, in press).

Along similar lines, another project partner, Dickinson College, wanted to know if there were differences on the BEVI between students who declared majors in different areas of study. If so, such differences also could have implications for different approaches to engagement and outreach, and help clarify the reasons why students were inclined toward different academic trajectories in the first place. In this analysis of 173 students, interest in global learning experiences was measured by the following question: "On a scale of 1-7, with 1 being 'extremely low' and 7 being 'extremely high,' please indicate your level of personal interest in international education or study abroad experiences." Students' academic majors were divided into four categories: social science, arts and humanities, "hard science," and undecided. A one-way ANOVA was conducted to determine if differences existed between students' interest in global learning experiences across multiple academic majors. As Table 8 indicates, no significant differences emerged, a finding that was somewhat unique among project participants. Thus, we examined the data more closely. Analysis of the means for each major indicates that all students at Dickinson College have a very strong interest in global experiences, regardless of academic major. Given the very strong historical and current commitment to global education expressed in Dickinson's mission statement, brand identity, and curriculum, such findings make good sense (i.e., at an institution like Dickinson, "interest" in international learning is not an especially good predictor of differences between students since most everyone coming to the institution does so with these interests in mind). In combination with other results, such findings ultimately led to another implication, that institutions and organizations have very different "profiles" or "signatures," which should be assessed in order to apprehend the nature and culture of its members (see Implication 11 below). 
Table 8

Interest in Global Learning Experiences ${ }^{1}$ by Students' Academic Major: Dickinson College

\begin{tabular}{lcccccc}
\hline Source & $\begin{array}{c}\text { Type II Sum } \\
\text { of Squares }\end{array}$ & Mean & df & $\begin{array}{c}\text { Mean } \\
\text { Square }\end{array}$ & $\mathrm{F}$ & Sig. \\
Corrected Model & $2.865 \mathrm{a}$ & & 3 & 0.955 & 0.688 & 0.561 \\
Intercept & 6605.555 & & 1 & 6605.56 & 4758.89 & 0.000 \\
Major & 2.865 & & 3 & 0.955 & 0.688 & 0.561 \\
$\quad$ Social sciences & & 6.385 & & & & \\
$\quad$ Arts and humanities & & 6.313 & & & \\
$\quad$ Sciences & 6.143 & & & \\
$\quad$ Undecided or other & 6.082 & & & \\
Error & 234.58 & & 169 & 1.388 & \\
$\quad$ Total & 6843 & & 173 & & & \\
$\quad$ Corrected Total & 237.445 & & 172 & & &
\end{tabular}

$\mathrm{R}^{2}=.012$ (Adjusted $\mathrm{R}^{2}=-.005$ )

${ }^{1}$ Global Learning Experiences = international, multicultural, and transformative learning experiences.

Notes. 1. Post hoc tests indicate there are no significant differences among the majors.

2. Items are measured on a 4-point scale (1=strongly disagree - 4=strongly agree)

Implication 6. All educators and educational experiences are not equal. The fact that all learning interventions and interveners are "not equal" should promote personal, professional, and institutional development and engagement, as well as a renewed commitment to improvement, rather than discouragement, defensiveness, or denial.

Although intuitively reasonable, and certainly a focus of emphasis within the academy, it would seem that there is a general consensus that those who teach, direct, or lead various learning experiences differ in their relative effectiveness. For example, the fact that we expend considerable energy evaluating the effectiveness of teaching faculty suggests an overarching recognition that such matters are integral to student success, course and program quality, and the pursuit of institutional and organizational goals. Such matters of teacher quality are not just of concern within higher education, but are central to all individuals who assume a teaching role at the K-12 level as well (e.g., Cultivating the Globally Sustainable Self, 2015; Ellis, Lee, \& Wiley 2009; InTASC, 2011; Welch, Pitts, Tenini, Kuenlen, \& Wood, 2010). Even so, for a variety of reasons, it is difficult to tease apart the relative impact of "teacher" from "learner" much less the interactions between the two that serve to mediate and moderate learning. Thus, one intriguing and consistent finding that we believe has long-term relevance is the apparent differences that emerged in learner outcomes as a function - at least in part - of who was teaching and/or leading a particular learning experience. For example, consider the following juxtaposition between two different Time 1 / Time 2 group report profiles by two different individuals who were responsible for leading the same learning experience (see Figure 3). How do we interpret such findings? First, it is important to appreciate that this juxtaposition is not based upon truly random assignment to either instructor. Rather, as is often the case with large required courses, these students simply were assigned to one or another section on the basis of a number of factors (e.g., their own course schedules; adviser decisions and enrollments), which are not controlled for in this convenience sample. Thus, any number of variables might have affected the results we observe. However, in this particular analysis, the basic process for assignment of students to various sections was not pre-determined (i.e., was more or less random in a non-experimental sense, in that there was no specific logic or plan to assignment of students to a specific section). More importantly, from our review of Time 1 / Time 2 BEVI reports across different institutions and programs or experiences, such results do not appear to be anomalous in that we repeatedly see substantial differences in Time 1/Time 2 reports across instructors or directors who are teaching or leading 
experiences that ostensibly are designed to be "the same." That said, the most fundamental point we offer in this regard - which is at the core of all we have observed throughout this project - is that we simply cannot understand who learns what and why, and under what circumstances, if we do not simultaneously take into account and assess multiple variables, including teacher characteristics (e.g., dispositions), student characteristics (e.g., life histories), and the quality and nature of the learning experience (e.g., how it is taught/delivered, where, and for what purposes) (e.g., Cultivating the Globally Sustainable Self, 2015). So, we recommend reviewing the patterns revealed in Figure 3 not so much as definitive, but rather as illustrative of the kinds of differences that we have observed, and that warrant further study in the years to come. So, in the context of these caveats, let's focus on a few key points of comparison. Specifically, consider differences in Figure 4 on Negative Life Events, Self Awareness, and Global Engagement across these two instructors.

Essentially, on these three Time 1/Time 2 comparisons, students of Instructor 1 report, by the conclusion of the course, that they had experienced a greater degree of Negative Life Events, are more Self Aware, and are more Globally Engaged. The exact opposite Time 1/Time 2 profile is associated with Instructor 2 (students report substantially fewer Negative Life Events, much less Self Awareness, and much less Global Engagement). Although hard and fast conclusions are not warranted for the reasons noted above, since the goals for each section of the course ostensibly were the same (e.g., to produce greater awareness of different cultures), these results should give pause at the very least, as they suggest students are reporting radically different experiences at the conclusion of the course.

Why? Among other possible hypotheses, the most parsimonious would seem to be the skill through which course content was conveyed and course experiences were facilitated as well as overarching fidelity to the course objectives that were pursued. Such matters of content and process are, of course, at the heart of teaching and learning. As our annual processes of faculty evaluations across higher education institutions indicate, we certainly believe that faculty differ in their instructional competencies, a supposition that would seem to receive at least potential support from such findings. At the same time, many other variables could account for such differences as well, including fundamental differences in the nature of various cohorts (e.g., most faculty who have taught for any duration are likely to experience substantial differences in the make up or nature of cohorts from semester to semester).

And yet, the dramatic difference in directionality from Time 1 to Time 2 across the above three scales and others - suggest that such phenomena should be studied more closely over time, particularly because we have observed such differences in outcomes across instructors, programs, and institutions. The results suggest not only a deep and largely neglected need to examine differences in the knowledge, skills, and dispositions of those who are in an "instructional" realm, broadly defined, but also how such findings may be used to promote growth and development of instructors and facilitators of change at multiple levels and in various sectors of the educational system. That is to say, if used with care and skill, such findings may help individual instructors understand better the characteristics of their group (i.e., through group reports of their students) as well as what is happening from the beginning to the conclusion of a learning experience. If skillfully facilitated, such findings should help teaching faculty reflect upon and cultivate capacities over the short- and long-term that are more likely to be effective in terms of facilitating learning, development, and change (e.g., Cultivating the Globally Sustainable Self, 2015). 
Instructor 1

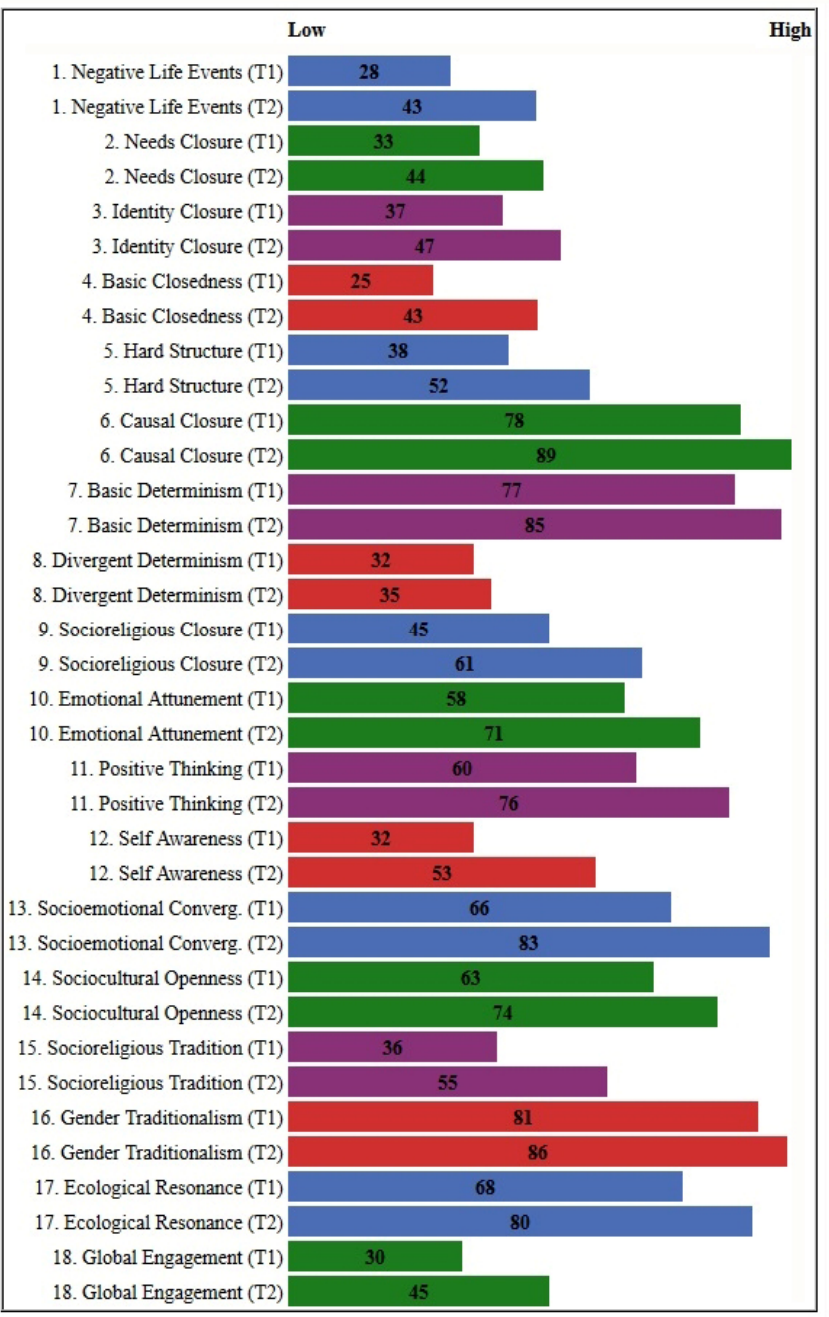

Instructor 2

\begin{tabular}{|c|c|c|}
\hline & Low & High \\
\hline 1. Negative Life Events (T1) & 32 & \\
\hline 1. Negative Life Events (T2) & 15 & \\
\hline 2. Needs Closure (T1) & 40 & \\
\hline 2. Needs Closure (T2) & 17 & \\
\hline 3. Identity Closure ( $\mathrm{T} 1$ ) & 36 & \\
\hline 3. Identity Closure (T2) & 12 & \\
\hline 4. Basic Closedness (T1) & 28 & \\
\hline 4. Basic Closedness (T2) & 9 & \\
\hline 5. Hard Structure (T1) & 39 & \\
\hline 5. Hard Structure (T2) & 12 & \\
\hline 6. Causal Closure (T1) & 81 & \\
\hline 6. Causal Closure (T2) & 50 & \\
\hline 7. Basic Determinism (T1) & 79 & \\
\hline 7. Basic Determinism (T2) & 54 & \\
\hline 8. Divergent Determinism (T1) & 17 & \\
\hline 8. Divergent Determinism (T2) & 7 & \\
\hline 9. Socioreligious Closure (T1) & 68 & \\
\hline 9. Socioreligious Closure (T2) & 41 & \\
\hline 10. Emotional Attunement (T1) & 63 & \\
\hline 10. Emotional Attunement (T2) & 49 & \\
\hline 11. Positive Thinking (T1) & 75 & \\
\hline 11. Positive Thinking (T2) & 18 & \\
\hline 12. Self Awareness ( $\mathrm{T} 1)$ & 36 & \\
\hline 12. Self Awareness (T2) & 8 & \\
\hline 13. Socioemotional Converg. (T1) & 81 & \\
\hline 13. Socioemotional Converg. (T2) & 54 & \\
\hline 14. Sociocultural Openness (T1) & 70 & \\
\hline 14. Sociocultural Openness (T2) & 53 & \\
\hline 15. Socioreligious Tradition (T1) & 56 & \\
\hline 15. Socioreligious Tradition (T2) & 38 & \\
\hline 16. Gender Traditionalism (T1) & 81 & \\
\hline 16. Gender Traditionalism (T2) & 52 & \\
\hline 17. Ecological Resonance (T1) & 68 & \\
\hline 17. Ecological Resonance (T2) & 47 & \\
\hline 18. Global Engagement (T1) & 39 & \\
\hline 18. Global Engagement (T2) & 7 & \\
\hline
\end{tabular}

Figure 3. Comparison of Time 1 and Time 2 profiles for two different instructors of the same course.

\begin{tabular}{|c|c|c|c|}
\hline 1. Negative Life Events (T1) & 28 & 1. Negative Life Events (T1) & 32 \\
\hline 1. Negative Life Events (T2) & 43 & 1. Negative Life Events (T2) & 15 \\
\hline 12. Self Awareness (T1) & 32 & 12. Self Awareness (T1) & 36 \\
\hline 12. Self Awareness (T2) & 53 & 12. Self Awareness (T2) & 8 \\
\hline 18. Global Engagement (T1) & 30 & 18. Global Engagement (T1) & 39 \\
\hline 18. Global Engagement (T2) & 45 & 18. Global Engagement (T2) & 7 \\
\hline
\end{tabular}

Figure 4. Comparison of $\mathrm{T} 1$ / $\mathrm{T} 2$ results for two different instructors on the same course across three BEVI scales.

Ultimately, ensuring instructor competence, effectiveness, and impact is an ever-relevant issue for institutions and organizations. We therefore suspect that using such rich data to analyze the quality of instructors, directors, and leaders could in fact result in significant improvements to course or program offerings over the long term. Moreover, if students or other learners repeatedly are exposed to instructors or directors who consistently achieve results that are below - or above - those of their colleagues, we have a very 
real opportunity to intervene in a data-driven manner in order to 1) promote the instructional, curricular, or programmatic quality of a specific learning experience; 2) encourage ongoing professional development that may be tracked over time; 3) recognize and explore "excellence" when it repeatedly occurs in terms of "better" learning processes outcomes, to understand what actually is happening in real time between teachers, trainers, and learners; and, 4) make changes when all else fails - and after all good faith attempts to improve matters so that learners are not repeatedly subjected to subpar instructors, directors, or leaders who do not appear to be facilitating acceptable learning processes and outcomes.

At the very least, these findings suggest the need to examine such outcome differences in order to understand what the interactions may be among teacher competencies, student characteristics, and the nature of various learning experiences that are designed to promote "change" (e.g., Cultivating the Globally Sustainable Self, 2015). The need to consider such matters becomes especially important when one realizes that exposure to international, multicultural, or transformative learning may not always result in "good" outcomes at Time 2 (see Implication 7). For example, we have observed Time 1/Time 2 instructional profiles which may show a much higher degree of Emotional Attunement and Self Awareness, but lower Sociocultural Openness, at Time 2. Contrast that outcome with its mirror opposite of a much lower degree of Emotional Attunement and Self Awareness at Time 2, but higher Sociocultural Openness. Which outcome is better? One's answer probably depends upon one's goals in terms of a course, program, or workshop, but arguably, a higher degree of Emotional Attunement and Self Awareness may actually be a function of exposure to content and experiences that are highly activating of the "self," which may not necessarily translate into greater openness toward the sociocultural "other" (e.g., Tabit et al., in press) at the conclusion of a learning experience. Over the long term, however, such an outcome may bode better than the opposite outcome, which finds our learner less Emotionally Attuned or Self Aware, but reporting more Sociocultural Openness. As always, the reasons for such discrepant outcomes should be a point of exploration and contemplation which may be much better interpreted and understood in the context of overall scale scores and qualitative findings - rather than a basis for hard and fast conclusions on their own. Along similar lines, and as explicated in Implication 7 below, we strongly recommend longitudinal analysis (e.g., Time 3, Time 4 administration), mainly to hone in on what is happening over time. That is because it may well be that an initial decrement on various scales represents the initial "reaction" to an overwhelming experience, which must be metabolized over time in order to ascertain longer-term effects.

Implication 7. Educational experiences are associated with desirable as well as undesirable processes and outcomes across a wide range of constructs that must be measured to be known. Depending upon the nature and quality of learning, growth, or development experiences - and the life histories, worldviews, and capacities of those who deliver and engage in them - measurable processes and outcomes may or may not be in the anticipated direction. Moreover, "change" may be occurring in ways that are and are not expected. If such processes and outcomes are not measured (e.g., because they aren't appreciated or seen as relevant, or because they are assumed to be happening as a matter of course), we may miss information that could in fact be most important about what actually is happening for learners, irrespective of what we think is, or should be, happening. Thus, as educators, researchers, and administrators, we should embrace the fundamentally experimental nature of our work. We may learn as much from what doesn't work as what does, but only if we measure a sufficient range of relevant constructs.

Oftentimes, understandably, the focus within the larger field of higher education, including international, multicultural, and transformative learning as a whole, is on "what works." Such an emphasis makes good sense, of course, since it behooves us to develop, implement, and promote learning experiences that demonstrably impact our students, institutions, and organizations in a value-added manner. But sometimes the most useful findings that emerged from the Forum BEVI Project involved learning experiences that didn't seem to accomplish their intended goals, especially when these unexpected results were discussed openly and productively by project partners. To take one example of several, students enrolled in a course 
Table 9

Degree of Worldview Shift

\begin{tabular}{|c|c|c|c|c|c|c|}
\hline \multirow[b]{2}{*}{ Scale } & \multicolumn{2}{|c|}{ Time } & \multirow[b]{2}{*}{$F$} & \multicolumn{2}{|c|}{ Gender } & \multirow[b]{2}{*}{$F$} \\
\hline & Pre & Post & & Female & Male & \\
\hline $\begin{array}{l}\text { Causal } \\
\text { Closure }\end{array}$ & 1.229 & 1.317 & $\begin{array}{c}7.780 \\
(1,133)^{\star \star}\end{array}$ & 1.196 & 1.351 & $\begin{array}{c}11.595 \\
(1,133)^{\star \star \star}\end{array}$ \\
\hline Basic Determinism & 1.718 & 1.856 & $\begin{array}{c}9.187 \\
(1,134)^{\star \star \star}\end{array}$ & 1.615 & 1.958 & $\begin{array}{c}19.196 \\
(1,134)^{\star \star \star}\end{array}$ \\
\hline $\begin{array}{l}\text { Emotional } \\
\text { Attunement }\end{array}$ & 3.013 & 3.133 & $\begin{array}{c}6.920 \\
(1,133)^{\star \star}\end{array}$ & 3.257 & 2.889 & $\begin{array}{c}13.581 \\
(1,133)^{\star \star \star}\end{array}$ \\
\hline $\begin{array}{l}\text { Global } \\
\text { Engagement }\end{array}$ & 2.647 & 2.579 & $\begin{array}{c}4.454 \\
(1,134)^{\star}\end{array}$ & 2.691 & 2.535 & $\begin{array}{c}7.662 \\
(1,134)^{\star \star}\end{array}$ \\
\hline
\end{tabular}

designed to produce greater sociocultural openness and appreciation for diversity ${ }^{15}$ were selected for an analysis aimed at better understanding the degree to which individuals do or do not benefit from such a learning experience. As described in the syllabus, the purpose of the course was to provide students with the necessary tools to understand and appreciate the diverse cultures that they will likely encounter throughout their personal and professional lives. The study employed a one-group pretest/posttest design across four sections of the course. Students $(\mathrm{N}=137)$ who chose to participate in the research project were provided with a username and password and asked to complete the long version (336-item) BEVI pretest at the beginning of the semester. At the conclusion of the course, students were again asked to complete the BEVI as a posttest measure. Analysis of pre-post data was undertaken across all BEVI scales using repeated measures analysis of variance (ANOVA). This study was exploratory in nature, and as such, research questions focused mainly on whether Time 1/Time 2 differences could be observed, and if so, on which BEVI scales. Given the goals of the course, it was anticipated that some changes would be observed - in a positive or desired direction - particularly on scales tapping greater Sociocultural Openness and Global Engagement.

As indicated in Table 9, results suggest that the course, although designed to facilitate learning outcomes such as tolerance for cultures different from one's own, appears to be associated with the opposite effect. More specifically, upon completion of the course, students appeared to be more rigid in their belief systems, more inclined to endorse simple causal attributions regarding why human beings do what they do, more emotionally aware and activated, and less open to developing a deeper engagement with other cultures than they had been at course entry. It may, of course, be mistaken to assume that these real-time/short-term correlations will bear out over the long-term. Moreover, it cannot be concluded that the course is "causing" these changes or even that greater "negative" changes might have emerged had the course not occurred. All that said, however, it is striking that theoretically desirable attributes (e.g., openness, engagement) diminished over the duration of the semester since such outcomes are antithetical to the explicit goals of this course. Moreover, it should be noted that additional qualitative data (e.g., responses on the three free-response items of the BEVI; course evaluations) corroborate these quantitative data, suggesting that at least some aspects of this particular course should perhaps be reconfigured to focus, for example, more on learning process and less on content delivery, especially at the outset of the course (e.g., see Tabit et al., in press).

This Time 1/Time 2 within-group analysis provided one of our project partners an opportunity to use the BEVI to gauge and compare the relative effectiveness of this required course. Combined with other data,

${ }^{15}$ Adapted from Tabit, Legault, Ma, \& Wan (in press) from Shealy, C.N. (Ed.) (in press). Making sense of beliefs and values. New York, NY: Springer Publishing.

(C)2015 The Forum on Education Abroad 
profile results may be used to improve this teaching and learning experience over time, modify extant courses, develop new approaches, and so on. Moreover, such results provide an opportunity for self-assessment and professional development, as group reports may help course instructors, program directors and administrators better understand the nature of their particular class or cohort and their own effectiveness as instructors. For example, as described below, the report system for the BEVI allows instructors to examine group characteristics in detail and to consider how such variables impact the ways in which course content is experienced, which offers an opportunity to modify and/or tailor one's approach to teaching in order to proactively respond to the dynamics of the larger group and its subgroups. Along similar lines, by tracking experience-based outcomes over time, it is possible to identify those educator/leader dispositions that appear to be most and least effective in terms of meeting teaching or program outcomes. Such information may be quite helpful in terms of understanding areas of focus for personal and professional development.

At the same time, as noted above, it also is important to appreciate the longitudinal nature of "change" as is suggested by the EI model (Shealy, in press). That is to say, "negative" results from a learning experience may be due to multiple factors, including but by no means limited to 1) the quality of instruction or facilitation, 2) the nature of a particular cohort, and/or 3) the fact that exposure to content and processes that are "disequilintegrating" may show up on the BEVI in one way at Time 2, but be completely different at Time 3. Research currently underway is following cohorts beyond a Time 2 administration in order to examine both longitudinal processes that occur over time (at Time 3 and beyond) and cross-sectional differences that may characterize cohorts across different years.

Consider, in this regard, Figure 5 below, which juxtaposes Time 1, Time 2, and Time 3 BEVI Group Report profiles for the same group of study abroad students $(\mathrm{N}=20)$. As this figure illustrates, although some aspects of the T1/T2 comparison move in a direction that presumably would be desirable at the conclusion of a study abroad experience (e.g., Self Certitude drops from the $42^{\text {nd }}$ to the $28^{\text {th }}$ percentile; Gender Traditionalism drops from the $38^{\text {th }}$ to the $27^{\text {th }}$ percentile), other scales move in a direction that presumably would not be desirable given the goals of the study abroad experience (e.g., Needs Fulfillment drops from the $54^{\text {th }}$ to the $39^{\text {th }}$ percentile; Self Awareness drops from the $78^{\text {th }}$ to the $63^{\text {rd }}$ percentile; and Sociocultural Openness drops from the $76^{\text {th }}$ to the $62^{\text {nd }}$ percentile). As noted in Figure 5, T1/T2 administrations occurred between 3-4 months after initial administration of the BEVI at the beginning of the study abroad experience. So, what happens if we let at least half-a-year pass before assessing these same students again? Here we see a highly intriguing flip on a number of key scales. For example, in Figure 5 below, consider three scales noted above that presumably went in the "opposite direction" of what reasonably could be anticipated (or at least hoped for) at Time 2: first, Scale 3. Needs Fulfillment (which measures openness to experiences, needs and feelings; deep care/sensitivity for self, others, and the larger world); second, Scale 11. Self Awareness (which measures a tendency toward introspection, acceptance of self-complexity, care for the human experience/condition, and tolerance of difficult thoughts/feelings); and third, Scale 15. Sociocultural Openness (which measures progressiveness/openness regarding a wide range of actions, policies, and practices in the areas of culture, economics, education, environment, gender, global relations, and politics). As illustrated below, on all three of these scales (among others that might be reviewed as well from Figure 5), results now solidly are in the direction of what might be anticipated (or hoped for) at the conclusion of a study abroad experience. How do we explain such findings? From an Equilintegration or EI perspective, the "7Ds" of belief/values transformation (see Implication 14) explicitly acknowledge that multiple factors interact simultaneously to determine how, for whom, and under what circumstances "change" occurs (Shealy, in press). Moreover, the greater the discrepancy between one's original "Formative Variables" and those to which one is exposed next (e.g., how the individual experiences a culture with which they are deeply familiar as compared to how that same individual experiences a different and unfamiliar culture), the greater the degree of potential "affective/cognitive shut down" occurs, as the "self" strives essentially to protect "its self" from the intensely experienced shock of such exposure, which may in qualitative terms be described as "amazing," but nonetheless exerts a toll; thus, the individual simply may need time and space to consolidate (อ2015 The Forum on Education Abroad 
and "make sense of beliefs and values" once again in a way that is "equilintegrated" into one's newly reorganized self. Thus, we see a Time 3 profile that largely is a mirror image of Time 2, and in some ways, a poignant reminder of what the human self endures as we all go about the business of living, which is felt that much more dramatically when experiencing "high impact" learning like study abroad (e.g., Iyer, 2013; Kuh, 2008).

From the standpoint of the BEVI and its report system, the basic point to keep in mind here is that the interface between the complexities of being human and the complexities of assessment require us to adopt measurement strategies that do not wag the dog; in other words, let us first be who we are, and then and only then, seek to capture the meaning of our experience vis-à-vis assessment (e.g., by assessing such evolving human phenomenology over time, rather than "just once," as such an approach is more likely to allow for our human complexity to be apprehended as it naturally manifests in the real world) (Shealy, in press). At the same time, project results suggest that we cannot assume that "negative" results at Time 2 are due simply to the "disequilintegrating" processes that may occur as a result of challenging learning, growth, and development experiences; it may well be that the two other variables noted above - relative instructional/facilitator quality and/or cohort differences - may in fact account for much of the variance in this regard (i.e., the fact that learners may report qualitatively different responses at Time 3 than at Time 2 should not automatically be cited to deflect a focus from instructor/course quality and/or variability among cohorts). In short, our results suggest that if we really want to understand why we observe the measurable results that we do over time, our commitment to assessment must occur over the long-term, and simultaneously be open to examining a wide range of interacting variables that may well have personal as well as programmatic and institutional/organizational implications. 
T1/T2/T3 Aggregate BEVI Profile from a Sample of Study Abroad Students

(3-4 Months between T1 and T2 Administrations; 10-22 Months between T1 and T3 Administrations) $\mathrm{N}=20$

1. Negative Life Events

2. Needs Closure

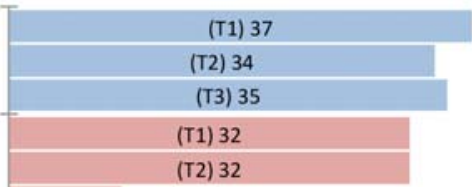

3. Needs Fulfillment

4. Identity Diffusion

5. Basic Openness

6. Self Certitude

$$
\text { (T3) } 9
$$$$
\text { (T2) } 32
$$
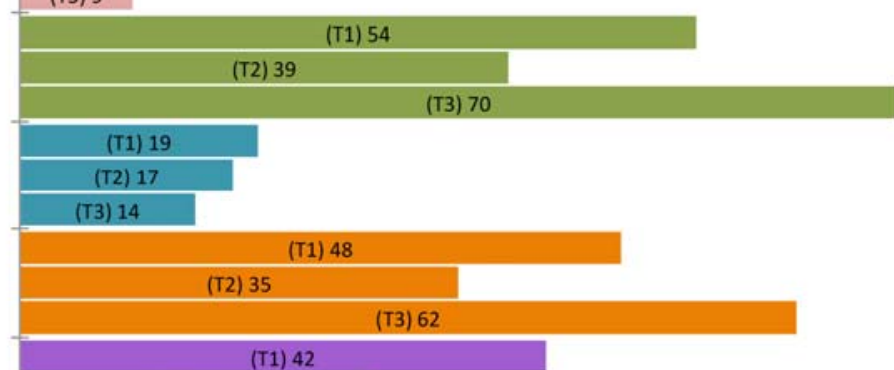

7. Basic Determinism

8. Socioemotional Convergence

9. Physical Resonance

10. Emotional Attunement

11. Self Awareness

12. Meaning Quest

13. Religious Traditionalism

14. Gender Traditionalism

15. Sociocultural Openness

16. Ecological Resonance

17. Global Resonance

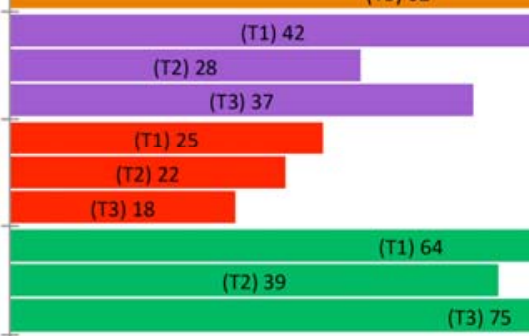

(T1) 82

(T2) 79

(T3) 80
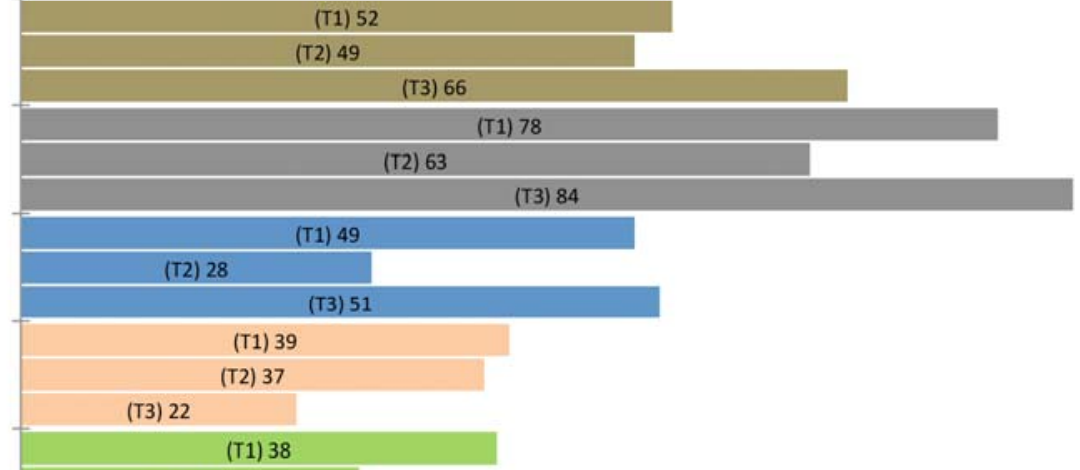

(T2) 27

(T3) 21

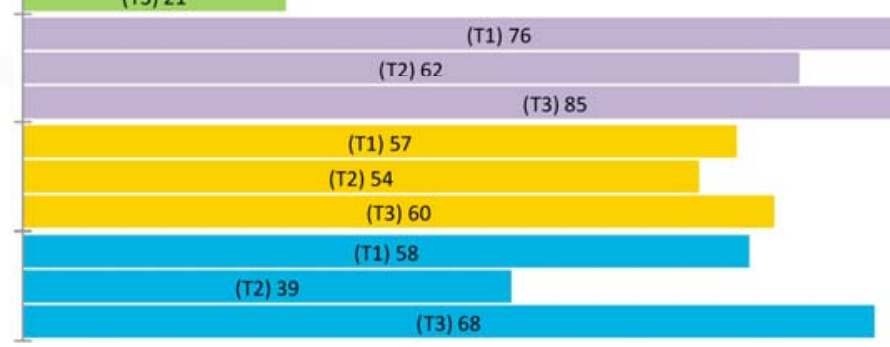

Figure 5. Comparison of $\mathrm{T} 1$ / $\mathrm{T} 2$ / $\mathrm{T} 3 \mathrm{BEVI}$ group report results for the same group of study abroad students. 
Implication 8. Examining only overall (e.g. aggregate) findings or privileging only a few BEVI scales constitute tempting but counter-productive approaches to comprehensive assessment. Aggregate findings can be misleading because they may mask or wash out "opposing" data for subgroups, which, when compiled, "cancel" each other out and become invisible. Thus, it is essential that the reporting system be able to account for change processes that may be occurring both within 1) demographic subsets of learners and 2) educators who are leading specific types of learning experiences. Likewise, ignoring some of the scales to focus on only a few (i.e., Sociocultural Openness) fails to recognize the rich and complex processes students undergo, the relationships between these processes, and the importance of assessing other aspects of the learning process, which may be as - if not more - important in explaining why we do, or do not, observe particular findings.

It was heartening early on in the Forum BEVI Project to see results which clearly illustrated not only that change was emerging from Time 1 to Time 2 administrations, but in ways that would be expected. Consider, for example, Figure 6 below, a Time 1/Time 2 analysis $(\mathrm{N}=19)$ from Brethren Colleges Abroad (BCA), which mirrors the Time 1/Time 3 Profile above from Figure 5, and emphasizes a related finding: it always is important to consider one's unique results in relation to the specific instructor, group, and context in which a learning, growth, or development experience occurs. In many ways, this profile of this small $\mathrm{N}-$ from students who experienced a range of study abroad programs in different locales - represents what might be seen as optimal. In addition to the interesting finding that 1 ) Negative Life Events goes down (from the $33^{\text {rd }}$ to the $25^{\text {th }}$ percentile), these students would appear 2) to be "clearer" about who they are and where they are going in life (Identity Closure decreases from the $45^{\text {th }}$ to the $30^{\text {th }}$ percentile); 3) to become more sophisticated and nuanced at the level of attributions made about self, others, and the larger world (e.g., Basic Determinism decreases from the $87^{\text {th }}$ to the $56^{\text {th }}$ percentile); 4) to demonstrate greater introspection and interest in "how people work" (Self Awareness increases from the $28^{\text {th }}$ to the $48^{\text {th }}$ percentile); 5) to report fewer stereotyped notions regarding who males and females are and should be (Gender Traditionalism decreases from the $73^{\text {rd }}$ to the $59^{\text {th }}$ percentile); and, 6) to evidence a greater degree of interest in encountering different cultures and practices around the world (Global Engagement increases from the $15^{\text {th }}$ to $39^{\text {th }}$ percentile), from Time 1 to Time 2. 


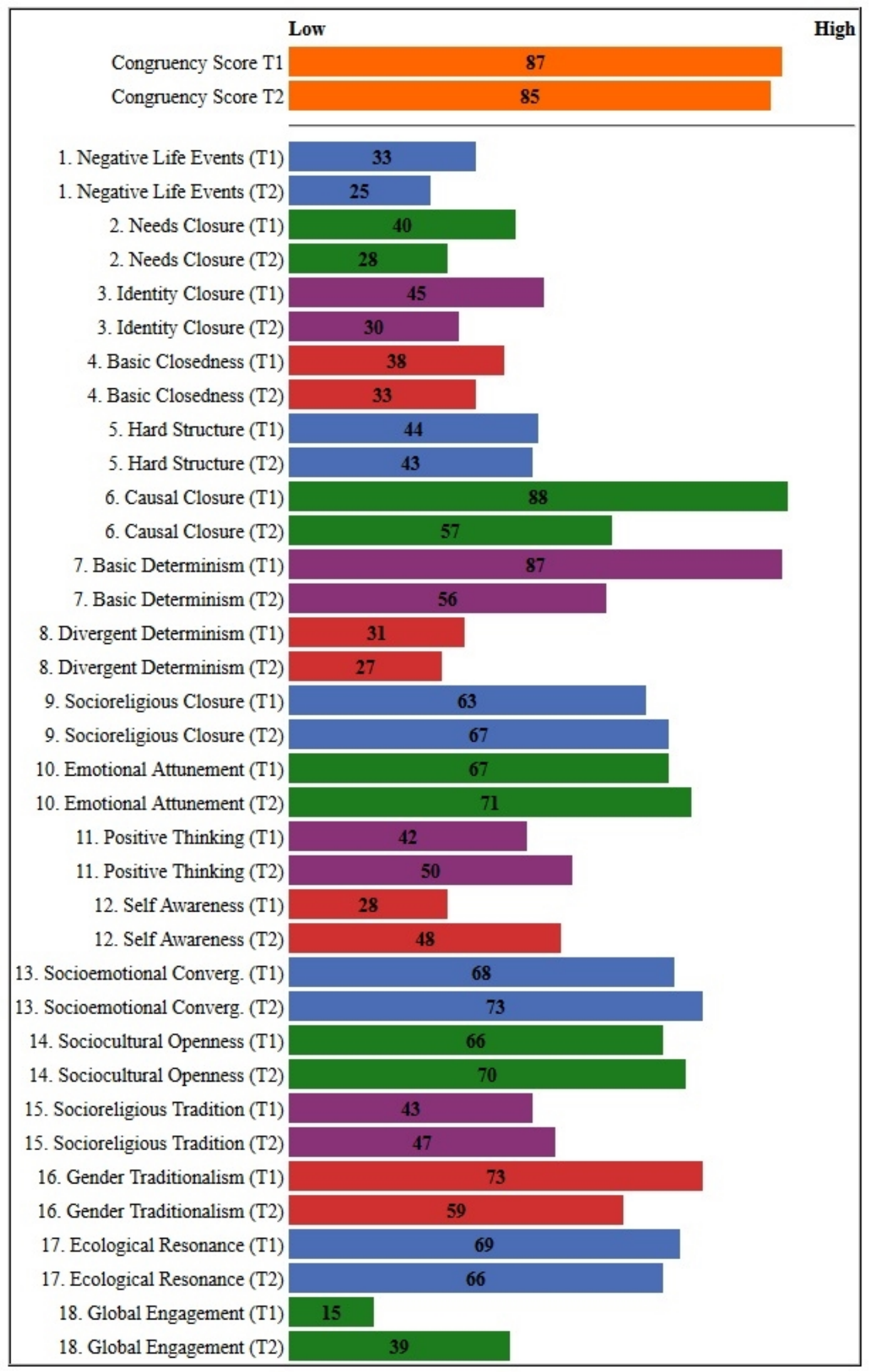

Figure 6. T1/T2 Group Report $(\mathrm{N}=19)$. 
Again, although such a profile may in fact be "optimal," a fundamental problem with such Time 1/Time 2 assessment analyses, no matter what measures are in use, is that such a report may obscure or wash out subgroup differences within the larger group. In other words, aggregate presentations of data do not allow for a more fine-grained analysis of what actually is happening within the group, not only at a scale level, but in terms of multiple mediators and moderators of change (e.g., background/demographic information) that may affect who learns what and why, and under what circumstances. As such, although interesting, a simple Time $1 /$ Time 2 analysis of the group as a whole for any assessment measure, including the BEVI, would probably not be sufficient to understand the complex and interacting within group factors that ultimately influence the nature and degree of any changes that were observed. Moreover, imagine a Time $1 /$ Time 2 profile that is the opposite of that pictured in Figure 6. Would we therefore conclude the learning experience was a complete failure? That could be the case, but at the very least, it would be helpful to do further statistical analysis to determine what exactly is happening within facets of the larger group. It could be, for example, that some groups may share similarities due to self-selection processes that might lead subsets of students to seek a study abroad or multicultural learning experience whereas others would not. In short, our assumption has been that multiple and substantial differences would characterize various subgroups within the larger group. Hundreds of analyses have confirmed the validity of this most basic proposition, which has substantive implications for the interpretation and application of BEVI Time 1/Time 2 reports, and, we would argue, for the very nature of how Time 1 and Time 2 evaluation of learning processes and outcomes occurs, regardless of which measure(s) are used.

Consider the following analysis, from one of our project partners, the University of British Columbia (UBC) in Canada. Here, in a series of Group Report juxtapositions $(\mathrm{N}=36)$, we are comparing Aggregate Profile results with those of Profile Contrast. By way of orientation, the description of each of these indices is as follows: ${ }^{16}$

Profile Contrast illustrates how different and similar the group is across all 17 BEVI scales via the lowest 30 percent, middle 40 percent, and highest 30 percent of full scale scores. The full scale score is summative of scores from the seven domains of the BEVI under which the 17 process scales are clustered: I. Formative Variables; II. Fulfillment of Core Needs; III. Tolerance of Disequilibrium; IV. Critical Thinking; V. Self Access; VI. Other Access; and, VII. Global Access (Shealy, in press). Profile Contrast is "key" to interpreting whether and to what degree groupings by low, medium, and high full scale scores are associated with different elevations on specific BEVI scales. For example, such information may be helpful in interpreting the variability within a specific group, which may range from minimal to substantial. Profile Contrast also may help users comprehend how and why subgroups within the larger group show changes in similar and different directions in the context of learning, growth, and development experiences. Thus, this index is considered a more robust and nuanced measure of the differential impact of specific learning experiences than are aggregated indices, which may obscure subgroup differences or cancel out changes that are in fact occurring among subsets of the larger group.

Aggregate Profile presents aggregated background information and domain scores for the full scale scores of the entire group (i.e., 1st - 100th percentile). In other words, this is the profile we are suggesting indeed is interesting and relevant (i.e., a version of this type of "aggregate profile" typically is relied upon when engaging in group-based Time 1/Time 2 assessment) - but should not be used alone. It is most useful for Time 1 assessment (i.e., understanding where the overall group is at the outset of a learning, growth, or development experience) and to track group results over time (i.e., from year to year). However, it generally should not be used alone to determine if a learning, growth, or development experience is or is not effective (e.g., at Time 2 assessment) mainly because it may obscure subgroup differences and/or cancel out changes

16 The following information for Aggregate Profile and Profile Contrast is excerpted and/or adapted from the "Description of Tables and Indices" section of BEVI Group Reports. 
that actually are occurring within various subsets of the larger group. From the standpoint of the BEVI, the best index to determine what is happening in this regard (i.e., between the lowest 30 percent, middle 40 percent, and highest 30 percent of full scale scorers) is Profile Contrast.

To illustrate these points, consider the following comparisons of Aggregate Profile and Profile Contrast results across five BEVI scales for this sample from UBC: Negative Life Events; Identity Diffusion; ${ }^{17}$ Self Certitude; Religious Traditionalism, and Sociocultural Openness. To facilitate interpretation, in figures $7-11$ below, we have juxtaposed the three Time 1/Time 2 scale scores for Profile Contrast (lowest 30\%, middle $40 \%$, and highest 30\%) with the Aggregate Profile score for each respective scale. In this way, it is possible to compare subgroup differences and similarities (Profile Contrast) with the overall score (Aggregate Profile) for each scale in this series of five separate analyses.

Consider Figure 7 below. In the first place, note that Aggregate Profile for Negative Life Events (NLE) goes up from the $48^{\text {th }}$ to the $55^{\text {th }}$ percentile from Time 1 to Time 2 . However, on Profile Contrast, the Lowest 30 percent and middle 40 percent (70 percent of the overall sample) actually go down from Time 1 to Time 2 on Negative Life Events. A basic and overarching conclusion from such results is that Profile Contrast is a much more robust and nuanced analysis of what is actually happening within a group than is the Aggregate Profile. So, we must be very careful in our interpretation of Time 1/Time 2 findings on any measure including but not limited to the BEVI - in order to ensure that we are apprehending what actually is happening within subsets of the overall group, as such subgroups may actually be responding very differently from one another as a result of exposure to an international, multicultural, or transformative learning experience.

Note also the very interesting finding - observed across multiple Group Report analyses - that the perception of one's life history and background may be differentially affected by one's point of departure prior to engaging in an international, multicultural, or transformative learning experience. This observation is highly congruent with a narrative framework that how we experience ourselves, others, and the larger world is mediated strongly by the life experiences we have and may not "exist" in absolute or inviolable terms (Shealy, in press). In other words, our memory and experience of our "past" are highly dependent upon events that occur in the present and future. And yet, this process may unfold differently for individuals depending upon their point of departure (i.e., how they are "organized" affectively or cognitively prior to the learning experience). For example, as Figure 7 illustrates, individuals who report a greater degree of "Negative Life Events" at Time 1 subsequently show a decrease in such a report at Time 2 (three to four months after the international/multicultural learning experience) whereas individuals who report a lesser degree of "Negative Life Events" at Time 1 subsequently show an increase in such a report at Time 2. What do such findings suggest? Although we emphasize that such findings are common, but not universal, these outcomes suggest that prolonged exposure to radically different "formative variables" (e.g., a different culture, context, language, religion, etc.) interacts with "who we were" at the outset of the experience to produce a sort of "progression to the mean" effect. Such findings imply further that those who experienced their past as especially "negative" or "positive" aren't so sure that that was the case following the completion of this intensive learning experience (i.e., relative to what they now have experienced via a program, course, workshop, etc., their past may now be experienced by them as "better" or "worse" than what they originally believed).

${ }^{17}$ For purposes of clarification, note that some scale names / results are from the "long" version of the BEVI whereas others are from the "short" version of the BEVI. For example, the name of this scale is "Identity Closure" on the long version, but "Identity Diffusion" on the short version. Additional chapters in Making Sense of Beliefs and Values (e.g., chapter 4) provide further clarification regarding processes by which the BEVI and its scales were developed (see Shealy, in press; http://www.springerpub.com/making-sense-of-beliefs-and-values.html). 


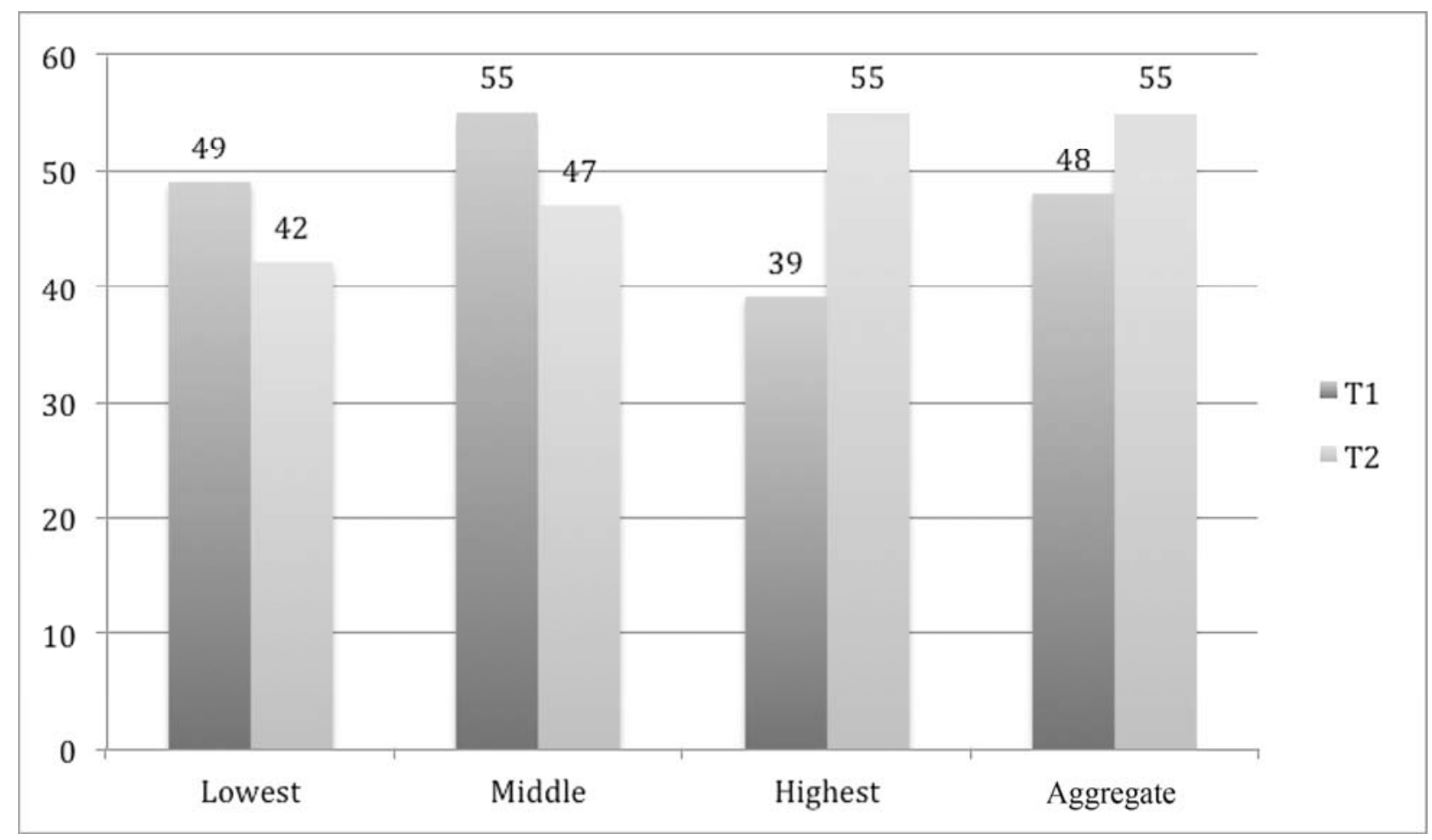

Figure 7. Comparison between lowest, middle, and highest Profile Contrast scores with the Aggregate Profile score on the Negative Life Events scale from the BEVI.

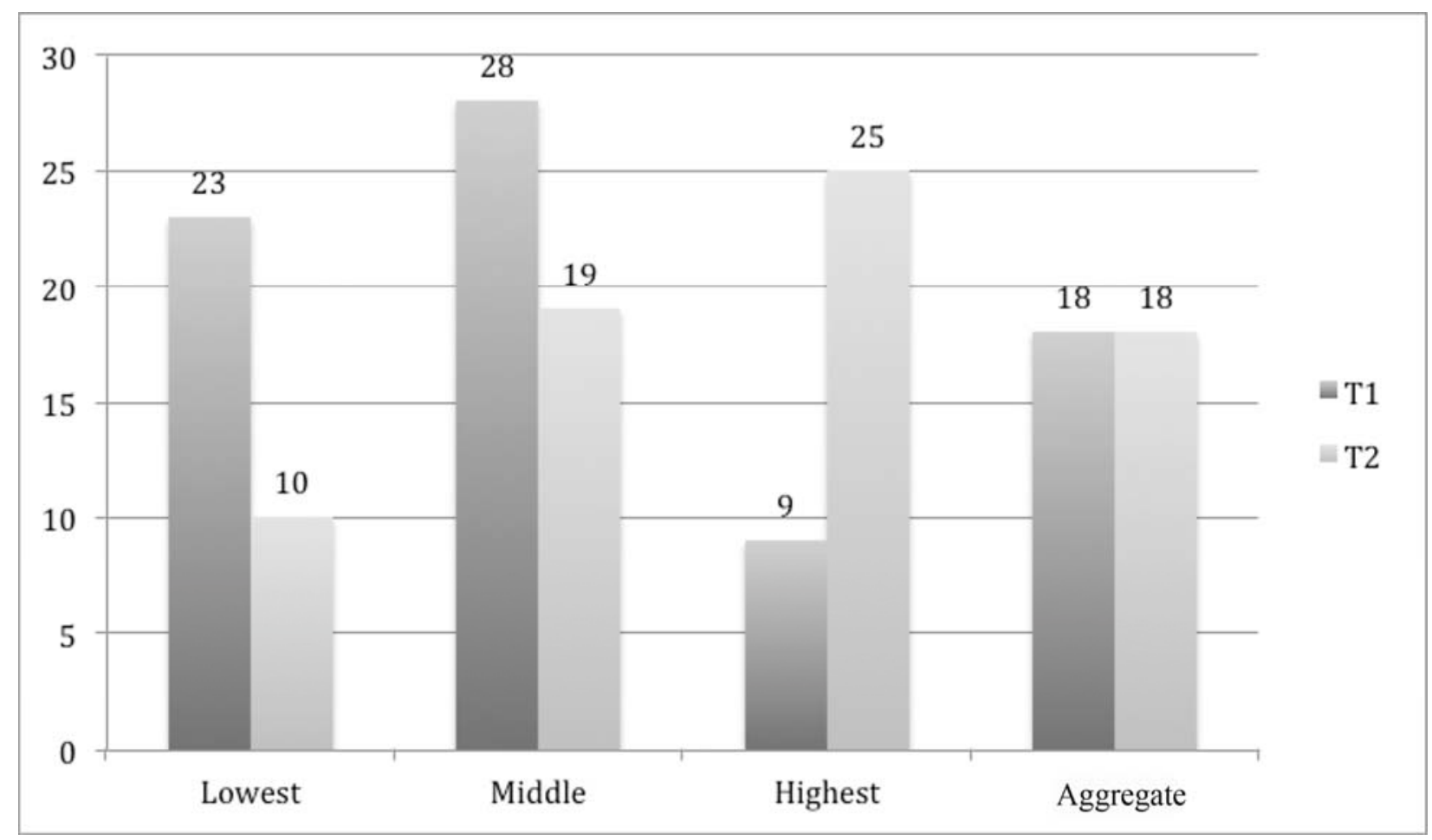

Figure 8. Comparison between lowest, middle, and highest Profile Contrast scores with the Aggregate Profile score on the Identify Diffusion scale from the BEVI. 
Consider Figure 8. From an interpretive standpoint, the Aggregate Profile and Profile Contrast results on Identity Diffusion amplify the above point. Recall that "Identity Diffusion" indicates the degree to which individuals report a "painful crisis of identity," and are "fatalistic regarding negatives of marital/family life," and "feel 'bad' about one's self and prospects." If we only reviewed the Time 1/Time 2 Aggregate Profile (which again, takes the average of all participants), we might conclude that no change occurred on this fundamental aspect of how one experiences self from the beginning to the conclusion of the experiences (i.e., the Time 1/Time 2 Aggregate Report remains at the $18^{\text {th }}$ percentile). Such a conclusion would be ill-advised as there are clear differences between subgroups on this core construct of the BEVI. Specifically, although none of these subgroups are elevated on this scale - an important point to consider vis-à-vis interpretation of the group as a whole - the lowest and middle "full scale" cohorts would appear to become substantially clearer on who they are and where they are going in life, whereas those who believed they were the clearest on such aspects of self at the beginning of the experience report markedly less clarity by the conclusion of this experience. The reasons for such findings warrant further exploration, but one possibility is that by dint of their exposure to a context and culture that are substantially different to what they were accustomed, each subgroup may "balance" what they previously believed and valued against what they saw in terms of the realities that others faced. Such a process may differentially be associated with greater clarity for those who were relatively unclear about who they were and where they were going versus those who, relatively speaking, felt clearer about such matters at the outset, but became less convinced of their clarity by the end of the experience.

Such tentative conclusions receive additional support via the following comparison between the Aggregate Profile and Profile Contrast for Self Certitude, which measures the degree to which individuals experience a "strong sense of will," are "impatient with excuses for difficulties," tend to emphasize "positive thinking," and are "disinclined toward deep analysis" regarding why they or others behave as they do or why events happen as they do in the larger world. As the findings of Figure 9 illustrate - and again, the group overall demonstrates a low degree of this construct - overall findings (from Aggregate Profile) illustrate that individuals report becoming less certain regarding such matters overall following an international, multicultural, or transformative learning event. However, very important differences - at least for this group - emerge, in that those who showed the highest "full scale" profile (i.e., were the most inclined to be open and engaged regarding the experience they were about to have) showed the greatest drop in certitude by the conclusion of their experience. The lowest full scale group (those that were least inclined) also showed a drop, whereas the middle 40 percent showed no change at all. Overall, it would appear that these students became "less certain" overall, although those in the middle appeared not to change on this core measure of certitude over the course of their experience. 


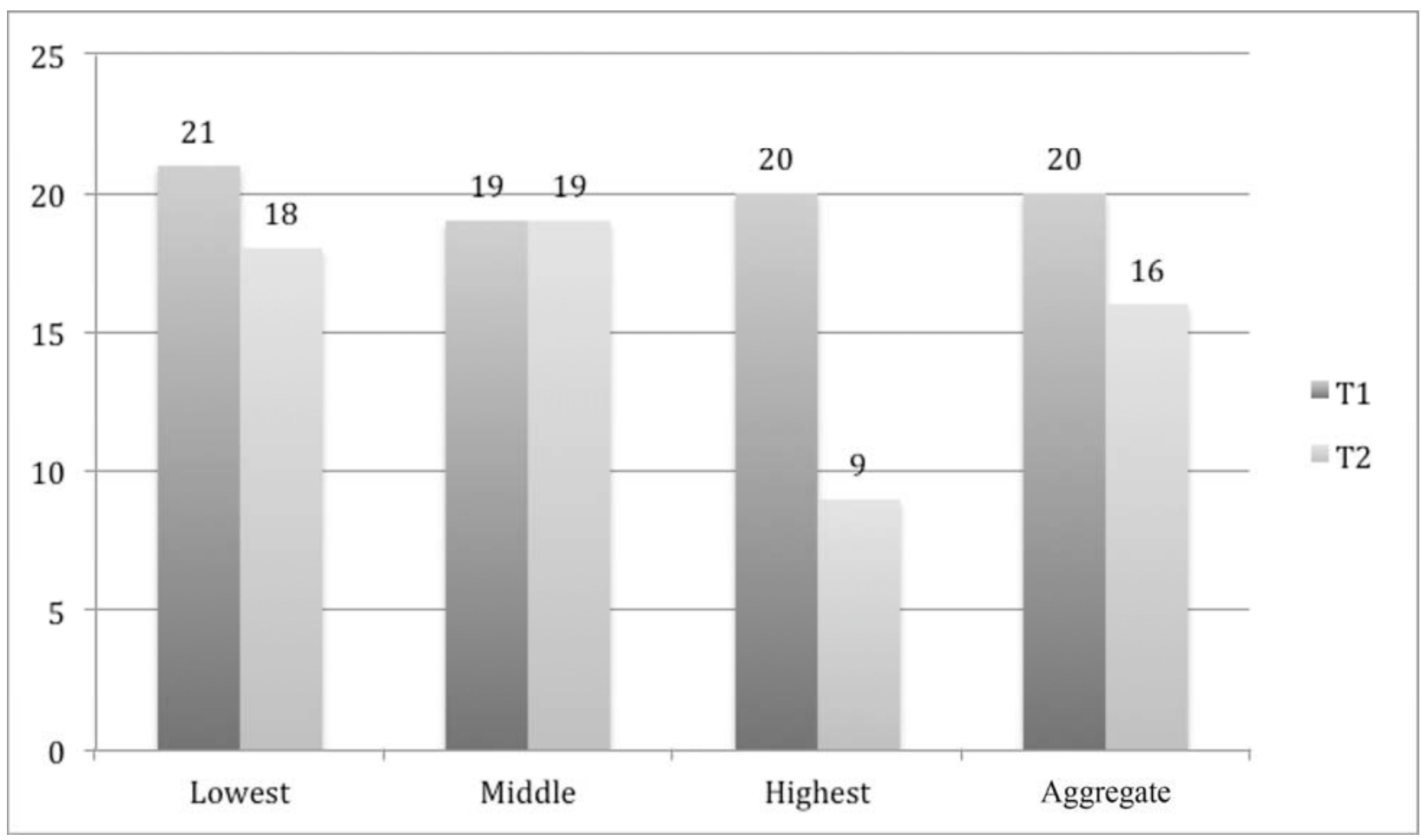

Figure 9. Comparison between lowest, middle, and highest Profile Contrast scores with the Aggregate Profile score on the Self Certitude scale from the BEVI.

Importantly, global matters of "certitude" may not apply equally to all belief/value constructs that are integral to one's larger worldview. For example, the following finding also has emerged on several occasions, and has to do with specific subsystems of belief. Consider Figure 10, which addresses the degree to which one is "highly religious," tends to see "self/behavior/events as mediated by God/spiritual forces," and contends that there is "one way to the 'afterlife'." Interestingly, here, the Aggregate Profile is a good representation of how the lowest and highest full scale subgroups respond, but does not reflect results for the middle 40 percent of this group. How do we interpret such findings? Although subject to further inquiry, a preliminary hypothesis is that individuals who are most invested in a traditional religious worldview experience the greatest challenge to the black and white nature of such a framework when exposed to beliefs and values that are markedly different from their own. Interestingly, such a finding does not correspond with an increase in "Identity Diffusion," as noted above (quite the contrary), which suggests that such transitions are not experienced as cognitively/affectively confusing in terms of why the world works as it does, but perhaps, are clarifying regarding who we are and who we are striving to become. 


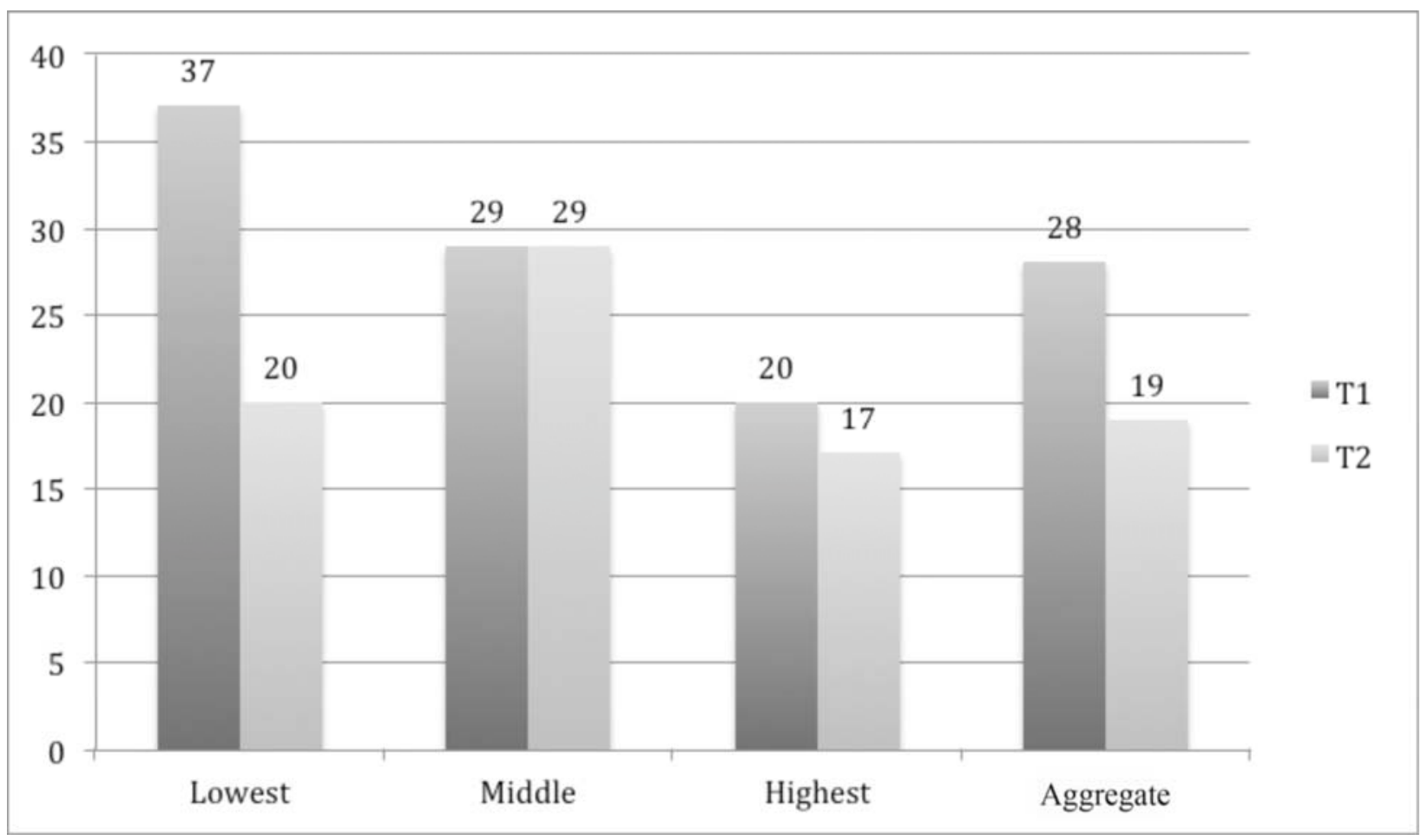

Figure 10. Comparison between lowest, middle, and highest Profile Contrast scores with the Aggregate Profile score on the Religious Traditionalism scale from the BEVI.

Finally, for those engaged in study abroad, service learning, multicultural education, or other educational interventions that are designed to facilitate greater openness to different ways of believing in or behaving around the world, the following findings, from Figure 11, are worth noting. Specifically, observe that this group of 36 students from the University of British Columbia would appear to be extremely socioculturally open at the beginning of their learning experience. Although there is a 10 percentage point difference at Time 1 between the lowest and highest full scale scores, it should be emphasized that this group really is "the choir," in that those engaged in trying to promote the beliefs, values, and practices of a "global citizenry" have a very ready audience. So, looking only at scales on the BEVI which measure such outcomes isn't the most relevant area of focus for this particular cohort. In other words, if our goal is "sociocultural openness," this group already is there at Time 1. 


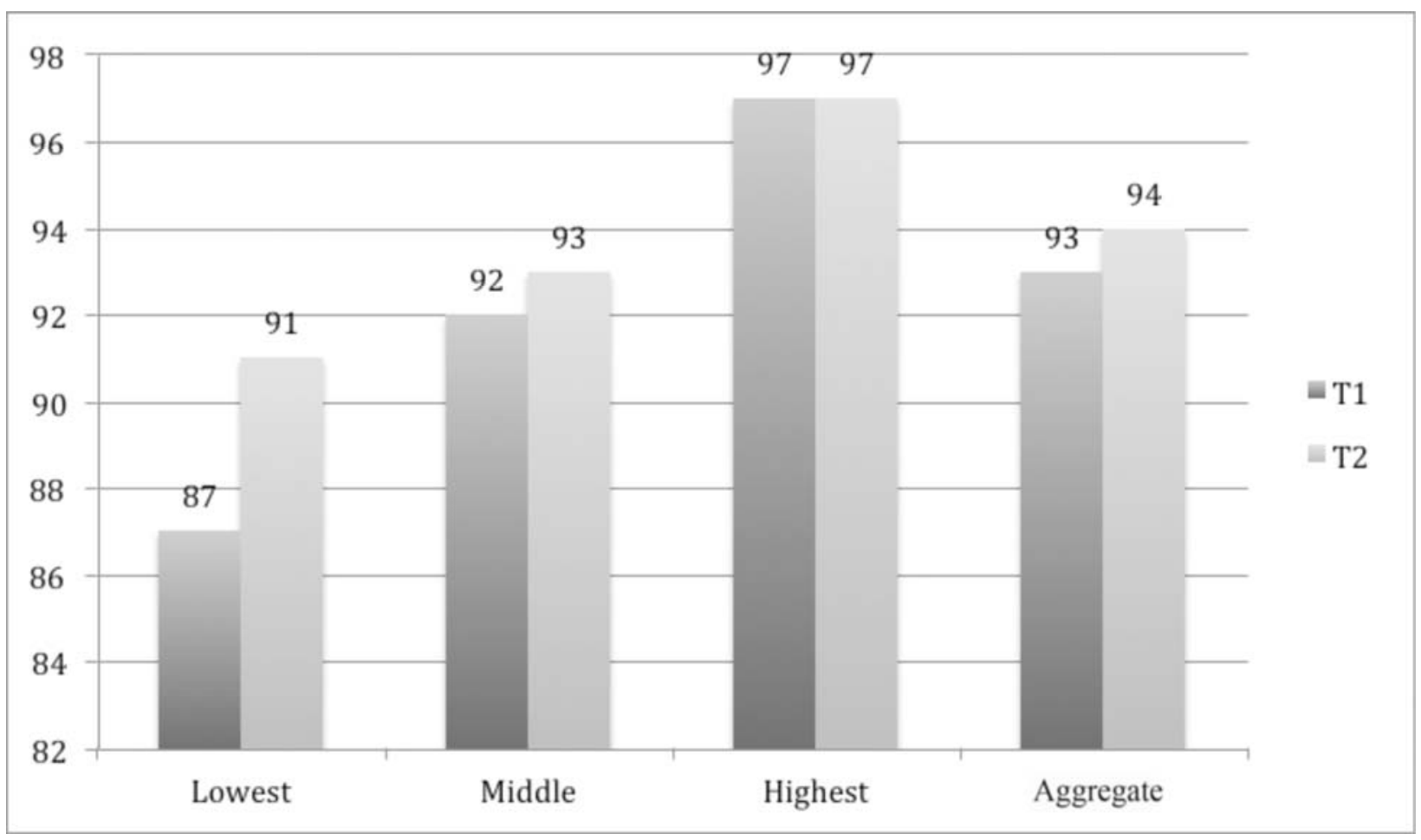

Figure 11. Comparison between lowest, middle, and highest Profile Contrast scores with the Aggregate Profile score on the Sociocultural Openness scale from the BEVI.

Thus, a fundamental recommendation from the Forum BEVI Project is to look far beyond traditional or expected indices of what we are trying to facilitate, and instead delve much more deeply into the complex interactions that mediate and moderate why and how we experience self, others, and the larger world as we do. That is, as international, multicultural, and transformative educators, scholars, and administrators, we need to include, but look beyond "sociocultural" beliefs, values, and practices in order to understand whether, how, for whom, and to what degree our learning experiences are impacting learners. A focus only on "intercultural" learning would miss the broader implications of the above findings, which illustrate that learners are being impacted at multiple levels of self (Shealy, in press). If we do not ask about these aspects of how self, others, and the larger world are experienced - to include but not be limited to "intercultural" beliefs and values - we miss apprehending the breadth and depth of all that we are impacting via the learning experiences we create. For example, if we only looked at Sociocultural Openness, it would be tempting to observe simply that, "Well, they were open at the beginning and are pretty much the same at the end." Such a simplistic conclusion ignores the rich changes that are in fact occurring - as the other four above comparisons reveal - as well as the complex and meaningful interactions that are taking place with subgroups of the larger group. The essential point here is that no specific construct should be privileged above any other, regardless of the primary educational goal we are seeking to promote. Just as our learners are not only "cultural learners," so should our assessment instruments measure much more than "cultural learning" alone.

Along these lines, we have quite often observed the intriguing finding that BEVI participants' reports of their own history become more "negative" over the course of a few weeks or months (between Time 1 and Time 2 administrations). This phenomenon may occur even while participants simultaneously report a greater degree of Emotional Attunement or Self-Awareness at the conclusion of the experience, which suggests two intriguing possibilities that are reminiscent of several implications above. First, consistent with the constructivist paradigm, it may well be that our present experiences alter our past perceptions under certain circumstances such as study abroad. Second, certain experiences may activate emotional arousal or promote self-awareness, but not increase, or even decrease, global engagement. Thus, if we only ask about "cultural" 
beliefs and values, we may well miss the most important - and perhaps the most interesting and relevant aspects of what actually is happening within our "learners" when they are exposed to different cultures. In short, we need to examine multiple "parts" and the overarching "whole" simultaneously in order to apprehend the experiential gestalt of learning, both at the individual and group level. In so doing, we are much more able to grasp the underlying interactions within and among various cohorts of learners, which will help us understand what is happening and why, and inform whatever decisions we make regarding changes or developments to pursue (e.g., curricular, staffing, faculty, programmatic, administrative, organizational, institutional).

As a final point under this implication, recall that the BEVI includes three "free response" qualitative questions - as a mixed methods measure - which allow respondents to offer their perspectives regarding matters of growth and change, or the lack thereof, in their own words. The importance of mixed methods approaches is well documented in terms of assessment best practice (e.g., Creswell \& Plano-Clark, 2007; Hanson, Leighton, Donaldson, Terjesen, \& Shealy, in press; Teddlie \& Tashakkori, 2009), the legitimacy of which has been confirmed to us as well on multiple occasions throughout the Forum BEVI Project. Qualitative responses provide a window into values-based similarities and differences among various cohorts regarding their initial or concluding expectations or perceptions regarding a learning experience. Such information may be very helpful in ensuring that program directors or course instructors are better attuned to what students or other participants are thinking and feeling vis-à-vis the learning experience, while using such information to facilitate more skillful programmatic or instructional interventions. Qualitative data also may provide key insight regarding why quantitative data emerge as they do. For example, when profile scores move in directions that are the opposite of what was expected or desired from Time 1 to Time 2 (suggesting that the overall experience may have been more "negative" than "positive"), qualitative responses are crucial to interpreting what may be happening, particularly if several students/participants report similar experiences. Specifically, when responses are relatively "neutral" or open at Time 1 (e.g., "I haven't had an international experience yet"; "I don't know, but look forward to the class") - and become indifferent or negatively toned upon Time 2 BEVI administration, at the conclusion of the experience (e.g., "I've had enough and can't wait to get home"; "This class was terrible") - we gather important information that may help explain the directionality of scale scores. Finally, along similar lines, qualitative responses both help explain quantitative findings and highlight the complexity of international, multicultural, and transformative learning processes and outcomes. For example, consider the following three prototypical response excerpts to qualitative question 3 on the BEVI: "What have you learned and how are you different as a result of this experience?":

- I realize how privileged I have been in my life.

- I now see why my country is the best place to live in the world.

- Who I thought I was is very different from what I think now.

When juxtaposed with quantitative scale scores, such responses not only represent a sort of phenomenological shorthand for how a student or participant has experienced a program, course, or workshop, but also offer a window into why response profiles may be what they are, particularly if patterns emerge across cohorts. For example, a student who concludes that they now know the "U.S. is best" likely had a very different experience and/or was a very different person at the outset of the experience than someone who now recognizes how "privileged" they are as compared to how they thought or felt at the beginning of the experience. Again, such responses illustrate a fundamental finding of this entire project: There are marked differences between students, faculty, directors, cohorts, and experiences - at the level of beliefs, values, and life experiences - that all interact together to influence the sort of "outcomes" that emerge at the conclusion of a learning experience. In short, qualitative data allow us to "make sense" of the complexity of international, multicultural, and transformative learning in ways that may be highly complementary to, and illuminating of, quantitative data. 
Implication 9. Although distinct characteristics of various cross-cultural groups are identifiable, broadbased assessment suggests within as well as between-group variation. A focus on differences between cultural groups is interesting and relevant, but may obscure the fact that greater variability may occur more often within, rather than between, different cultural groups. Such a conclusion has important implications for how we understand "human nature" and make sense of why we become who we become.

One major question of relevance to the BEVI Working Group was the overarching issue of whether and how this measure could be used in countries and cultures different from the United States. That is because the adaptation and usage of "Western" tests and measures in Non-Western contexts presents a range of challenges, which center largely on equivalency issues and underlying epistemologies of tests and measures developed in the U.S., which may or may not be consonant with those of other countries and cultures. From our perspective, there need not be an ineluctable schism between North/South, Western/non-Western methods and measures. Instead, our "differences" offer a fascinating and rich opportunity for us all to listen and learn from each other, ultimately responding in a collaborative, consensus-based manner that is inclusive, pluralistic, and culturally sensitive as well as methodologically and psychometrically defensible via assessment research and practice (e.g., Hanson et al., in press; Harkness, 2003; Moghaddam, Harre, \& Lee, 2007; Wild, Grove, Martin, Eremenco, McElroy, Verjee-Lorenz, \& Erikson, 2005).

Consistent with such a perspective, five points should be emphasized vis-à-vis cross-cultural usage of the BEVI. First, many BEVI items actually were identified outside of the U.S. in the context of clinical, research, and educational work with a highly diverse international population (Shealy, 2004). Second, every effort has been made throughout the development and evaluation of this measure to be mindful of cross-cultural issues of usage and application, and to strive for "equivalency" of usage across cultures and contexts. Multiple Subject Matter Experts (SME) have reviewed BEVI items at different points in the development of this instrument, including non-English language first/non-U.S. experts in international, multicultural, and transformative learning in order to identify possible issues of bias and/or non-relevance across countries and cultures. Third, from a theoretical perspective, the BEVI deliberately strives for neutrality regarding the "rightness" or "wrongness" of responses to BEVI items, allowing respondents to answer on a four-point Likerttype scale. ${ }^{18}$ As such, the fact that respondents from a "different" culture or context might tend to respond one way or another to various subsets of BEVI items, or even the BEVI as a whole, is precisely what the measure is designed to detect (i.e., differences and similarities between various subgroups). In other words, if such differences emerged across cultures and contexts, the most parsimonious explanation for such differences would be that the BEVI is detecting such belief/value differences rather than being biased toward, or against, one group or another. Fourth and similarly, based upon hundreds of analyses thus far, there is reason to believe that within group variation on the BEVI will be equivalent or greater than between group variation. For example, as the Decile Profiles below illustrate, although some culturally salient patterns may emerge on the BEVI, which are indeed relevant and meaningful (i.e., on beliefs/values regarding gender, religion, etc.), the general tendency is for broad-based populations (i.e., from a country) to show greater within than between group variation on many of the constructs that the BEVI measures. Fifth, in the final analysis, such matters ultimately are empirical in nature as we have to determine whether there are systematic differences at a population level that inadvertently skew results one way or another. Although further analysis of such issues remains ongoing, evidence thus far suggests that when administered and interpreted in an appropriate manner, the BEVI indeed appears able to be used across cultures and contexts to identify how various groups - even with very different life histories and worldviews - experience self, others, and the larger world. ${ }^{19}$

${ }^{18}$ See chapter 4 in Making Sense of Beliefs and Values (Shealy, in press) for a discussion of the design and structure of the BEVI, including the rationale for not including an "undecided" or "neutral" option.

${ }^{19}$ Reasons for cultural differences and similarities between groups are considered in several chapters in Making Sense of Beliefs and Values (e.g., see Tabit et al., in press). 
Consider, for example, the following three Decile Profiles that are drawn from students in three different countries/global regions under the auspices of universities that were part of the Forum BEVI Project. ${ }^{20}$ If there are radical differences across these three cultures, they presumably would manifest in patterns of responding across various scales on the BEVI (i.e., would be markedly different and/or skewed in various directions). Take a moment to review these three profiles and the dispersion of responses across the deciles for various BEVI scales.

Table 10

Three BEVI Decile Profiles from Students in Three Different Countries/Regions

\section{Country / Region 1}

\begin{tabular}{|c|c|c|c|c|c|c|c|c|c|c|}
\hline 1. Negative Life Events & $17 \%$ & $20 \%$ & $13 \%$ & $8 \%$ & $6 \%$ & $14 \%$ & $3 \%$ & $6 \%$ & $9 \%$ & $4 \%$ \\
\hline 2. Needs Closure & $54 \%$ & $15 \%$ & $9 \%$ & $7 \%$ & $3 \%$ & $3 \%$ & $3 \%$ & $3 \%$ & $1 \%$ & $1 \%$ \\
\hline 3. Needs Fulfillment & $2 \%$ & $2 \%$ & $3 \%$ & $7 \%$ & $6 \%$ & $11 \%$ & $9 \%$ & $20 \%$ & $19 \%$ & $22 \%$ \\
\hline 4. Identity Diffusion & $36 \%$ & $18 \%$ & $17 \%$ & $0 \%$ & $11 \%$ & $0 \%$ & $0 \%$ & $6 \%$ & $3 \%$ & $9 \%$ \\
\hline 5. Basic Openness & $6 \%$ & $8 \%$ & $6 \%$ & $11 \%$ & $7 \%$ & $11 \%$ & $15 \%$ & $8 \%$ & $18 \%$ & $12 \%$ \\
\hline 6. Self Certitude & $12 \%$ & $9 \%$ & $10 \%$ & $14 \%$ & $9 \%$ & $9 \%$ & $9 \%$ & $15 \%$ & $6 \%$ & $7 \%$ \\
\hline 7. Basic Determinism & $20 \%$ & $17 \%$ & $19 \%$ & $9 \%$ & $8 \%$ & $9 \%$ & $7 \%$ & $2 \%$ & $5 \%$ & $4 \%$ \\
\hline 8. Socioemotional Convergence & $1 \%$ & $4 \%$ & $3 \%$ & $5 \%$ & $10 \%$ & $7 \%$ & $11 \%$ & $14 \%$ & $23 \%$ & $23 \%$ \\
\hline 9. Physical Resonance & $0 \%$ & $0 \%$ & $0 \%$ & $1 \%$ & $1 \%$ & $5 \%$ & $3 \%$ & $13 \%$ & $58 \%$ & $20 \%$ \\
\hline 10. Emotional Attunement & $6 \%$ & $3 \%$ & $7 \%$ & $9 \%$ & $8 \%$ & $17 \%$ & $9 \%$ & $13 \%$ & $18 \%$ & $10 \%$ \\
\hline 11. Self Awareness & $1 \%$ & $0 \%$ & $0 \%$ & $1 \%$ & $1 \%$ & $3 \%$ & $18 \%$ & $8 \%$ & $27 \%$ & $42 \%$ \\
\hline 12. Meaning Quest & $4 \%$ & $3 \%$ & $13 \%$ & $8 \%$ & $8 \%$ & $10 \%$ & $8 \%$ & $20 \%$ & $10 \%$ & $14 \%$ \\
\hline 13. Religious Traditionalism & $0 \%$ & $25 \%$ & $18 \%$ & $6 \%$ & $18 \%$ & $8 \%$ & $8 \%$ & $8 \%$ & $6 \%$ & $4 \%$ \\
\hline 14. Gender Traditionalism & $24 \%$ & $15 \%$ & $18 \%$ & $5 \%$ & $7 \%$ & $9 \%$ & $7 \%$ & $6 \%$ & $3 \%$ & $5 \%$ \\
\hline 15. Sociocultural Openness & $2 \%$ & $3 \%$ & $1 \%$ & $3 \%$ & $6 \%$ & $6 \%$ & $9 \%$ & $13 \%$ & $11 \%$ & $46 \%$ \\
\hline 16. Ecological Resonance & $5 \%$ & $2 \%$ & $6 \%$ & $7 \%$ & $15 \%$ & $15 \%$ & $11 \%$ & $9 \%$ & $8 \%$ & $23 \%$ \\
\hline 17. Global Resonance & $2 \%$ & $5 \%$ & $6 \%$ & $8 \%$ & $0 \%$ & $7 \%$ & $7 \%$ & $13 \%$ & $35 \%$ & $17 \%$ \\
\hline Deciles: & 1 & 2 & 3 & 4 & 5 & 6 & 7 & 8 & 9 & 10 \\
\hline
\end{tabular}

${ }^{20}$ The Decile Profile essentially divides up each scale on the BEVI into chunks of ten percent - deciles - ranging from the basement to the ceiling of each scale. In so doing, Decile Profile illustrates the percentage of individuals from the larger group that fall into each decile, from the lowest $(1-10 \%)$ to the highest $(90-100 \%)$, for each BEVI scale. Also, for interpretive purposes, the darker the shade of gray, the greater the percentage of individuals who cluster in a particular decile. 


\section{Country / Region 2}

\begin{tabular}{|c|c|c|c|c|c|c|c|c|c|c|}
\hline 1. Negative Life Events & $9 \%$ & $17 \%$ & $4 \%$ & $9 \%$ & $9 \%$ & $17 \%$ & $13 \%$ & $13 \%$ & $9 \%$ & $0 \%$ \\
\hline 2. Needs Closure & $30 \%$ & $13 \%$ & $9 \%$ & $9 \%$ & $13 \%$ & $4 \%$ & $13 \%$ & $0 \%$ & $9 \%$ & $0 \%$ \\
\hline 3. Needs Fulfillment & $0 \%$ & $0 \%$ & $13 \%$ & $13 \%$ & $4 \%$ & $9 \%$ & $9 \%$ & $30 \%$ & $9 \%$ & $13 \%$ \\
\hline 4. Identity Diffusion & $17 \%$ & $9 \%$ & $4 \%$ & $0 \%$ & $4 \%$ & $0 \%$ & $0 \%$ & $26 \%$ & $13 \%$ & $26 \%$ \\
\hline 5. Basic Openness & $13 \%$ & $30 \%$ & $0 \%$ & $22 \%$ & $9 \%$ & $4 \%$ & $13 \%$ & $0 \%$ & $9 \%$ & $0 \%$ \\
\hline 6. Self Certitude & $0 \%$ & $4 \%$ & $0 \%$ & $9 \%$ & $9 \%$ & $17 \%$ & $9 \%$ & $17 \%$ & $0 \%$ & $35 \%$ \\
\hline 7. Basic Determinism & $4 \%$ & $17 \%$ & $22 \%$ & $13 \%$ & $9 \%$ & $9 \%$ & $9 \%$ & $4 \%$ & $0 \%$ & $13 \%$ \\
\hline 8. Socioemotional Convergence & $0 \%$ & $0 \%$ & $17 \%$ & $4 \%$ & $9 \%$ & $17 \%$ & $0 \%$ & $9 \%$ & $26 \%$ & $17 \%$ \\
\hline 9. Physical Resonance & $0 \%$ & $0 \%$ & $0 \%$ & $13 \%$ & $4 \%$ & $30 \%$ & $0 \%$ & $39 \%$ & $13 \%$ & $0 \%$ \\
\hline 10. Emotional Attunement & $4 \%$ & $4 \%$ & $13 \%$ & $13 \%$ & $26 \%$ & $9 \%$ & $4 \%$ & $13 \%$ & $13 \%$ & $0 \%$ \\
\hline 11. Self Awareness & $0 \%$ & $0 \%$ & $0 \%$ & $13 \%$ & $9 \%$ & $13 \%$ & $9 \%$ & $4 \%$ & $30 \%$ & $22 \%$ \\
\hline 12. Meaning Quest & $0 \%$ & $4 \%$ & $17 \%$ & $4 \%$ & $0 \%$ & $17 \%$ & $4 \%$ & $30 \%$ & $0 \%$ & $22 \%$ \\
\hline 13. Religious Traditionalism & $0 \%$ & $0 \%$ & $4 \%$ & $4 \%$ & $0 \%$ & $9 \%$ & $17 \%$ & $17 \%$ & $17 \%$ & $30 \%$ \\
\hline 14. Gender Traditionalism & $13 \%$ & $4 \%$ & $13 \%$ & $4 \%$ & $13 \%$ & $30 \%$ & $0 \%$ & $9 \%$ & $0 \%$ & $13 \%$ \\
\hline 15. Sociocultural Openness & $0 \%$ & $0 \%$ & $0 \%$ & $4 \%$ & $4 \%$ & $0 \%$ & $0 \%$ & $9 \%$ & $26 \%$ & $57 \%$ \\
\hline 16. Ecological Resonance & $0 \%$ & $4 \%$ & $9 \%$ & $0 \%$ & $9 \%$ & $13 \%$ & $17 \%$ & $9 \%$ & $17 \%$ & $22 \%$ \\
\hline 17. Global Resonance & $0 \%$ & $0 \%$ & $4 \%$ & $4 \%$ & $0 \%$ & $9 \%$ & $17 \%$ & $13 \%$ & $39 \%$ & $13 \%$ \\
\hline Deciles: & 1 & 2 & 3 & 4 & 5 & 6 & 7 & 8 & 9 & 10 \\
\hline
\end{tabular}


Country / Region 3

\begin{tabular}{|c|c|c|c|c|c|c|c|c|c|c|}
\hline 1. Negative Life Events & $4 \%$ & $25 \%$ & $12 \%$ & $12 \%$ & $4 \%$ & $12 \%$ & $4 \%$ & $12 \%$ & $8 \%$ & $4 \%$ \\
\hline 2. Needs Closure & $50 \%$ & $8 \%$ & $17 \%$ & $12 \%$ & $8 \%$ & $0 \%$ & $0 \%$ & $0 \%$ & $0 \%$ & $4 \%$ \\
\hline 3. Needs Fulfillment & $0 \%$ & $8 \%$ & $4 \%$ & $12 \%$ & $12 \%$ & $4 \%$ & $12 \%$ & $29 \%$ & $8 \%$ & $8 \%$ \\
\hline 4. Identity Diffusion & $8 \%$ & $29 \%$ & $21 \%$ & $0 \%$ & $21 \%$ & $0 \%$ & $0 \%$ & $12 \%$ & $8 \%$ & $0 \%$ \\
\hline 5. Basic Openness & $0 \%$ & $17 \%$ & $4 \%$ & $29 \%$ & $4 \%$ & $4 \%$ & $12 \%$ & $8 \%$ & $8 \%$ & $12 \%$ \\
\hline 6. Self Certitude & $4 \%$ & $25 \%$ & $4 \%$ & $17 \%$ & $0 \%$ & $17 \%$ & $4 \%$ & $21 \%$ & $8 \%$ & $0 \%$ \\
\hline 7. Basic Determinism & $21 \%$ & $12 \%$ & $0 \%$ & $21 \%$ & $4 \%$ & $12 \%$ & $12 \%$ & $4 \%$ & $4 \%$ & $8 \%$ \\
\hline 8. Socioemotional Convergence & $4 \%$ & $4 \%$ & $8 \%$ & $0 \%$ & $21 \%$ & $8 \%$ & $21 \%$ & $8 \%$ & $21 \%$ & $4 \%$ \\
\hline 9. Physical Resonance & $0 \%$ & $0 \%$ & $0 \%$ & $4 \%$ & $0 \%$ & $25 \%$ & $8 \%$ & $38 \%$ & $21 \%$ & $4 \%$ \\
\hline 10. Emotional Attunement & $0 \%$ & $4 \%$ & $21 \%$ & $17 \%$ & $8 \%$ & $8 \%$ & $21 \%$ & $12 \%$ & $4 \%$ & $4 \%$ \\
\hline 11. Self Awareness & $0 \%$ & $0 \%$ & $0 \%$ & $17 \%$ & $4 \%$ & $8 \%$ & $12 \%$ & $8 \%$ & $25 \%$ & $25 \%$ \\
\hline 12. Meaning Quest & $12 \%$ & $4 \%$ & $21 \%$ & $12 \%$ & $8 \%$ & $4 \%$ & $8 \%$ & $21 \%$ & $8 \%$ & $0 \%$ \\
\hline 13. Religious Traditionalism & $4 \%$ & $33 \%$ & $12 \%$ & $12 \%$ & $17 \%$ & $8 \%$ & $8 \%$ & $4 \%$ & $0 \%$ & $0 \%$ \\
\hline 14. Gender Traditionalism & $17 \%$ & $17 \%$ & $21 \%$ & $0 \%$ & $17 \%$ & $12 \%$ & $4 \%$ & $4 \%$ & $8 \%$ & $0 \%$ \\
\hline 15. Sociocultural Openness & $0 \%$ & $0 \%$ & $0 \%$ & $4 \%$ & $4 \%$ & $8 \%$ & $17 \%$ & $12 \%$ & $12 \%$ & $42 \%$ \\
\hline 16. Ecological Resonance & $0 \%$ & $4 \%$ & $8 \%$ & $17 \%$ & $25 \%$ & $8 \%$ & $4 \%$ & $4 \%$ & $12 \%$ & $17 \%$ \\
\hline 17. Global Resonance & $0 \%$ & $8 \%$ & $4 \%$ & $0 \%$ & $0 \%$ & $4 \%$ & $8 \%$ & $17 \%$ & $50 \%$ & $8 \%$ \\
\hline Deciles: & 1 & 2 & 3 & 4 & 5 & 6 & 7 & 8 & 9 & 10 \\
\hline
\end{tabular}

Since we're dealing with different countries and regions, it makes sense to look first at Sociocultural Openness, which measures a tendency toward "progressive" and "open" beliefs and values regarding "a wide range of actions, policies, and practices in the areas of culture, economics, education, environment, gender/global relations, politics." Are there differences across these three groups? Consider the percentage of the sample that falls in the tenth decile (e.g., at the $90^{\text {th }}$ percentile or higher on this scale): Country $1=46$ percent; Country $2=57$ percent; Country $3=42$ percent. Clearly, participants from all three of these countries/regions are extremely high on Sociocultural Openness as measured by the BEVI. How about a couple of other variables, which might reflect underlying cultural dynamics/issues, such as Religious Traditionalism and Gender Traditionalism? What do we see at this level? Essentially, it would appear that Country/Region 2 is the most "conservative" or "traditional" regarding matters of religion and gender. Specifically, 30 percent of Country/Region 2 participants fall in the $90^{\text {th }}$ percentile on Religious Traditionalism compared to four percent for Country/Region 1 and zero percent for Country/Region 3. And, there is much wider dispersion on Gender Traditionalism for Country/Region 2 than either 1 or 3, which generally show low levels of Gender Traditionalism. Does it help to know that these BEVI cohorts are comprised of individuals from the United States and Central America as well as a highly internationalized group studying in Japan? From the standpoint of religion and gender, which of these three cultures comprised of students in higher education (i.e., not the population as a whole) would likely tend to be more "conservative" or "traditional" on average from the standpoint of "formative variables" (e.g., cultural norms)? If you answered Central America, you are correct. Specifically, Country/Region 1 is from project partner Dickinson College in the United States, and consists mainly of U.S. citizens $(\mathrm{N}=158)$. Country/Region 2 is from project partner Georgetown University, which administered the BEVI to English-speaking students drawn from El Salvador and Honduras $(\mathrm{N}=23)$. Country/Region 3 is from project partner Hiroshima 
University, which administered the BEVI to an older, highly educated, and diverse cohort of English-speaking students which were studying in Japan $(\mathrm{N}=24)$.

Along similar lines, consider another BEVI Group Report analysis of U.S. citizens who have never studied abroad with a separate group of non-U.S. citizens/dual citizens who all completed the BEVI. Interestingly, on the majority of BEVI scales, these respondents - mostly college-aged students - showed similar scale results on most BEVI scales (i.e., within 5 points of each other). However, as Figure 12 illustrates, on three of these scales, differences of over 10 percentage points emerged which would seem to make sense - and perhaps be predicted - in terms of the capacity of the BEVI to differentiate between these groups. Specifically, the "Non-U.S. Citizen/Dual Citizen" group showed the following BEVI scale elevations relative to the "U.S. Respondents who Have Not Studied Abroad": Sociocultural Openness (75 versus 58); Ecological Resonance (65 versus 51); and Global Resonance (56 versus 45). At least on these comparisons, it appears that the BEVI is able to identify similarities and differences that are consistent with an intuitive understanding of what would be expected from the standpoint of validity. In short, on scales that are designed most directly to measure "other" and "larger world" concerns, it should not be surprising that the BEVI identified group-based differences that differentiate individuals who, on average, would more likely tend to be non-globally oriented at present (e.g., U.S. citizens who have not studied abroad) with those who likely have had more globally-oriented experiences overall relative to their U.S. counterparts (e.g., nonU.S./dual citizens).

U.S. Citizens Who Nave Not Studied Abroad

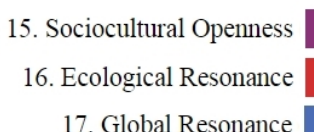

17. Global Resonance

\author{
16. Ecological Resonance \\ 17. Global Resonance
}

15. Sociocultural Openness
Non-U.S./Dual Citizens

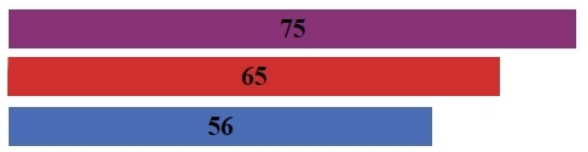

Figure 12. Comparisons between U.S. citizens who have not studied abroad and non-U.S. citizens/dual citizens.

The point of these two juxtapositions - three Decile Profiles with relatively small sample sizes as well as larger sample sizes across U.S. and non-U.S./dual citizens - is not to suggest that such matters of cross-cultural usage are settled, only that - consistent with the processes through which it was developed and evaluated over many years - the BEVI 1) does appear able to identify within group differences across each of these cultures, illustrating the belief/value diversity therein; and 2) findings that emerged are generally consistent with what would be expected for participants drawn across different cultural and demographic configurations. Of course, as we continue to gather data across cultures and contexts, further analyses will be conducted at a number of levels (i.e., whether reliable differences and similarities emerge across different countries and regions). Such efforts are consistent with other big picture and longitudinal assessment initiatives, such as the exemplary World Values Survey (Inglehart, Basáñez, \& Moreno, 1998; Inglehart, Basáñez, Díez-Medrano, Halman, \& Luijkx, 2004).

Implication 10. As Kurt Lewin observed, there is nothing so practical as a good theory. The importance of thinking about why learning, growth, or development does - or does not - occur cannot be overemphasized. By specifying what our theoretical understandings and commitments are, and are not, we are compelled to contemplate what our underlying (and often implicit) models and epistemologies are, which bear directly on fundamental questions of assessment models and methods. Without considering such matters, we risk engaging in assessment research and practice that is face valid and superficial as well as lacking in substantive, practical, and real world impact, mainly because we are, perhaps inadvertently, minimizing or ignoring the complexity of these interacting phenomena.

Although we rely on EI Theory and the EI Self in this project- mainly because they are integral to the 
BEVI - the overarching point is that whatever assessment approach is pursued, the underlying theory should be specified in detail in order to explicate why particular research and practice methods or interventions are recommended and/or specific interpretations justified. In addition to providing guidance regarding why we contend that particular interventions should impact learning, growth, and development in particular ways, it simply is not possible to conduct high-level types of statistical analyses without a strong theoretical basis for doing so. For example, structural equation modeling requires us to specify the proposed theoretical relationship among various measurable constructs, and was integral to the illumination of various associations among formative variables (i.e., life history, ethnicity), mediators (i.e., various BEVI process-oriented scales), and outcomes (i.e., reported satisfaction upon the conclusion of a learning experience) reported throughout this article. Among other benefits, a strong theoretical basis for our measures and methods allows us to

distinguish between descriptive and interpretive levels of analysis. That is, we need to examine both what constitutes change (e.g., how we know it is happening), a descriptive level of analysis, as well as why it is occurring (e.g., the rationale for why the changes we observe are in fact occurring), an interpretive level of analysis. We may be wrong or incomplete in our conclusions, but there can be no substitute for engaging in ongoing interpretation or explanation of why we observe the changes - or lack thereof - that we do (Shealy, in press).

Implication 11. It is possible to identify the profile or signature of an institution or organization. The aggregate profile of the BEVI may illuminate how a student body at a given institution or organization tends to see self, others, and the larger world. This profile appears to be relatively consistent across years, indicating that the characteristics of an institution's student body may be relatively stable and measurable.

One of the more intriguing findings from the Forum BEVI Project was the emergence of unique BEVI "profiles" or "signatures" that appear to characterize the student body across various institutions or organizations. It is perhaps not surprising that different systems tend to attract different students. Such findings are in fact a central preoccupation of colleges and universities, which keenly are interested in a range of defining characteristics of their student bodies (e.g., entering GPA, SATs/ACTs, ethnicity, etc.). The fact that such differences extend beyond these standard indices, and seem to characterize deeper aspects of the aggregate psychological make-up of a student body, is a unique and thought-provoking insight. Consider for example Figure 13, which juxtaposes aggregate scores over a two year period (2010 and 2011) across all BEVI scales for a stratified random sample of over 600 incoming freshman students from Michigan State University (MSU). Such results illustrate at least two basic findings. First, MSU students demonstrate remarkable continuity on the BEVI across two separate years of administration. In addition to offering further support for the reliability of the measure, what is most intriguing is that the entering MSU student body seems to show a very high degree of consistency in how they experience self, others, and the larger world across different years. Such findings should be of interest at an administrative level, since the student body not only shows stability at the level of traditional indices (i.e., GPA), but also at the level of underlying life history and psychological variables, such as Emotional Attunement and Sociocultural Openness. Such findings are not mere abstractions, but bear significantly on the overarching "culture" or "feel" of a campus community. More importantly, they may illuminate areas for focus vis-à-vis student programming, communication, and engagement (e.g., in academic affairs, student affairs, etc.), and may help in the interpretation or understanding of various events and dynamics that occur on campus. For example, during one of the years of this study, a racially charged incident occurred at MSU. While the larger community (both on and off campus) engaged in much deliberation regarding whether or not the student body as a whole was "racist," it was possible to observe - at least on the basis of these results - that it does not appear that the overall student body at MSU is inclined toward prejudicial beliefs; on the contrary, for the two years of this analysis, the entering MSU student body appears to be highly open toward and accepting of cultural differences (e.g., as illustrated by scores on Sociocultural Openness in the $71^{\text {st }}$ and $73^{\text {rd }}$ percentile in 2010 and 2011). However, a more fine-grained analysis of BEVI data by decile (e.g., through a Decile Profile) illustrates that there may 
well be small subsets of MSU students who are inclined toward racially prejudicial beliefs. Such findings not only put the matter in perspective vis-à-vis the student body as a whole, but may allow for more nuanced and targeted interventions, when appropriately designed and delivered. For example, it is quite common for colleges and universities to implement "sensitivity training" on various issues following incidents and/or as a proactive measure. Group based data of this sort may allow faculty, staff, and administrators to appraise more accurately the nature and scope of such problems rather than designing and implementing broad-based, onesize-fits-all interventions that may, inadvertently, do harm - rather than and/or in addition to - good (e.g., Tabit et al., in press).

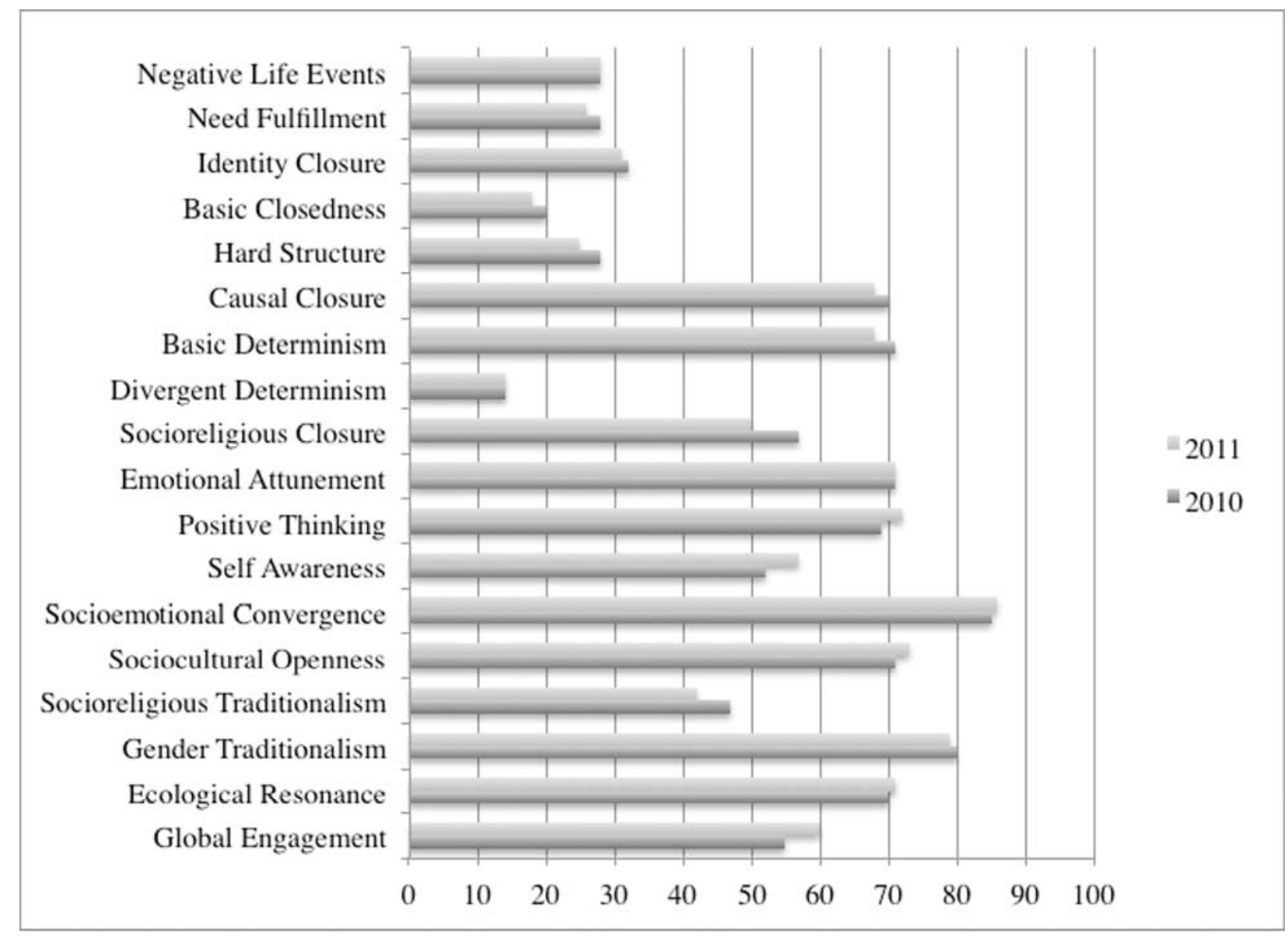

Figure 13. Stratified random sample of two years of entering freshman students at Michigan State University across all BEVI scales.

Second and consistent with the above observations, such results offer the possibility of understanding the aggregate make-up of the student body as a whole. More specifically, and focusing on a few key findings in Figure 13 across 2010 and 2011, MSU students overall appear not to report a high degree of unhappy life experiences prior to matriculation (Negative Life Events $=28^{\text {th }}$ percentile for both 2010 and 2011), and to be quite open/non-defensive generally (Basic Closedness $=20^{\text {th }}$ and $18^{\text {th }}$ percentile for 2010 and 2011). At the same time, the level of sophistication regarding "why humans do what they do" is not especially elevated at the outset of their higher education career (e.g., Basic Determinism $=71^{\text {st }}$ and $68^{\text {th }}$ percentile for 2010 and 2011), which is perhaps not surprising for students who are about to begin an intensive process of examining their underlying assumptions about the nature of reality. Auguring well over the long term in this regard, these students also evidence a high capacity for seeing the world in "shades of gray" rather than black and white (e.g., Socioemotional Convergence $=85^{\text {th }}$ and $86^{\text {th }}$ percentile for 2010 and 2011). Although such openness may not 
necessarily extend to beliefs and values about gender roles (e.g., Gender Traditionalism $=80^{\text {th }}$ and $79^{\text {th }}$ percentile for 2010 and 2011), they appear very moderate overall regarding their religious inclinations (e.g., Socioreligious Traditionalism $=47^{\text {th }}$ and $42^{\text {nd }}$ percentile for 2010 and 2011). Moreover, they do appear highly open to different cultural beliefs and practices (e.g., Sociocultural Openness $=71^{\text {st }}$ and $73^{\text {rd }}$ percentile for 2010 and 2011) and are highly concerned about the environment and natural world (Ecological Resonance $=70^{\text {th }}$ and $71^{\text {st }}$ percentile for 2010 and 2011). Taken as a whole, such a profile offers important insights about the aggregate psychological makeup of the MSU student body, particularly when compared to the aggregate profile of institutions with quite different missions and traditions. In short, then, the generation of institutional or organizational profiles would seem to have at least five specific usages:

1. Establishing a baseline for each cohort of entering students, which may be followed over time (through re-administration) in order to understand which aspects of the learning experience are associated with the most and least change.

2. Examining interactions among demographic and background variables (e.g., income, acceptance rates), specific learning experiences (e.g., study abroad, participation in a learning community), and outcomes (e.g., BEVI scale changes, GPA).

3. Developing and defining a "brand" (e.g., the relative openness of the student body), which allows for more precise strategic planning (e.g., "We wish to recruit more of X type of student in order to move the institution or organization in particular directions over time.").

4. Helping the institution or organization understand better who and what it is (e.g., what the normal distributional for the student body is as well as areas of greater and lesser variability).

5. Moving beyond traditional demographic tracking by understanding deeper aspects of its identity as an institution or organization, including how the student body experiences self, others, and the larger world.

Implication 12. Group reports help course instructors, program directors, and administrators understand better the nature of their particular class, cohort, or staff. Across a range of different settings and contexts, group reports have been used to help those in a position of leadership understand who their group is, which may help in planning and orientation purposes. Such reports also help assess what may be driving underlying processes of conflict or consensus for the group, which may result in more informed and sensitive leadership interventions. Further administration may allow for an examination of how the group sees itself, others, and the larger world over the long-term after particular interventions and/or experiences occur.

Transformational learning stands as an overarching goal of education writ large; yet the question needs to be asked: to what end and for what purpose (e.g., Cultivating the Globally Sustainable Self, 2015)? In cases where students will engage with vulnerable communities and complex topics, it is critical to establish the clear premise that student learning is not the only consideration; we also must ensure that students are engaging real issues and real people in a substantive manner. Understanding the signature profile of the institution generally - and the group profile of a cohort specifically - can assist instructors and program staff in making ethically sound decisions throughout the process of selection, preparation, monitoring, and support.

One typical usage of the report system in this regard is to compare and contrast various subgroups within a larger institution or organization in order to understand how change occurs over time, while also appreciating the differences and similarities among such groups. Such analysis is important since even strong trends for a group as a whole should not obscure differences within the group as a number of comparative reports from this overall initiative reveal. Consider for example Figure 14, which contrasts incoming freshmen students at Michigan State University (MSU) with a selected group of student leaders, who are especially adept at addressing issues of diversity and multiculturalism (the "Diversity Group"). Presumably, if the BEVI is able to detect differences between groups both currently and across time, substantive differences should emerge in the profiles of these groups. In full recognition that we are examining different groups with 
different Ns, a number of intriguing differences do exist and are worth considering as we contemplate future research. For example, there is a much higher degree of Negative Life Events amongst the Diversity group as compared to the Pre-entry group (Pre-entry $=28^{\text {th }}$ percentile; Diversity $=44^{\text {th }}$ percentile). Moreover, the Diversity group showed a much higher degree of attributional sophistication as evidenced by Basic Determinism (Pre-entry $=70^{\text {th }}$ percentile; Diversity $=30^{\text {th }}$ percentile) and a much lower degree of Gender Traditionalism (Pre-entry $=80^{\text {th }}$ percentile; Diversity $=42^{\text {nd }}$ percentile) as well as a higher degree of Self Awareness (Pre-entry $=52^{\text {nd }}$ percentile; Diversity $=69^{\text {th }}$ percentile) and Sociocultural Openness (Pre-entry $=$ $71^{\text {st }}$ percentile; Diversity $=83^{\text {rd }}$ percentile). The point is, although it is very relevant and informative to look at characteristics of the student body as a whole, it is no less important and illuminating to examine differences among subgroups within the broader population, both to understand differences and similarities between such groups and to map how various cohorts of students become "who they are" over time.
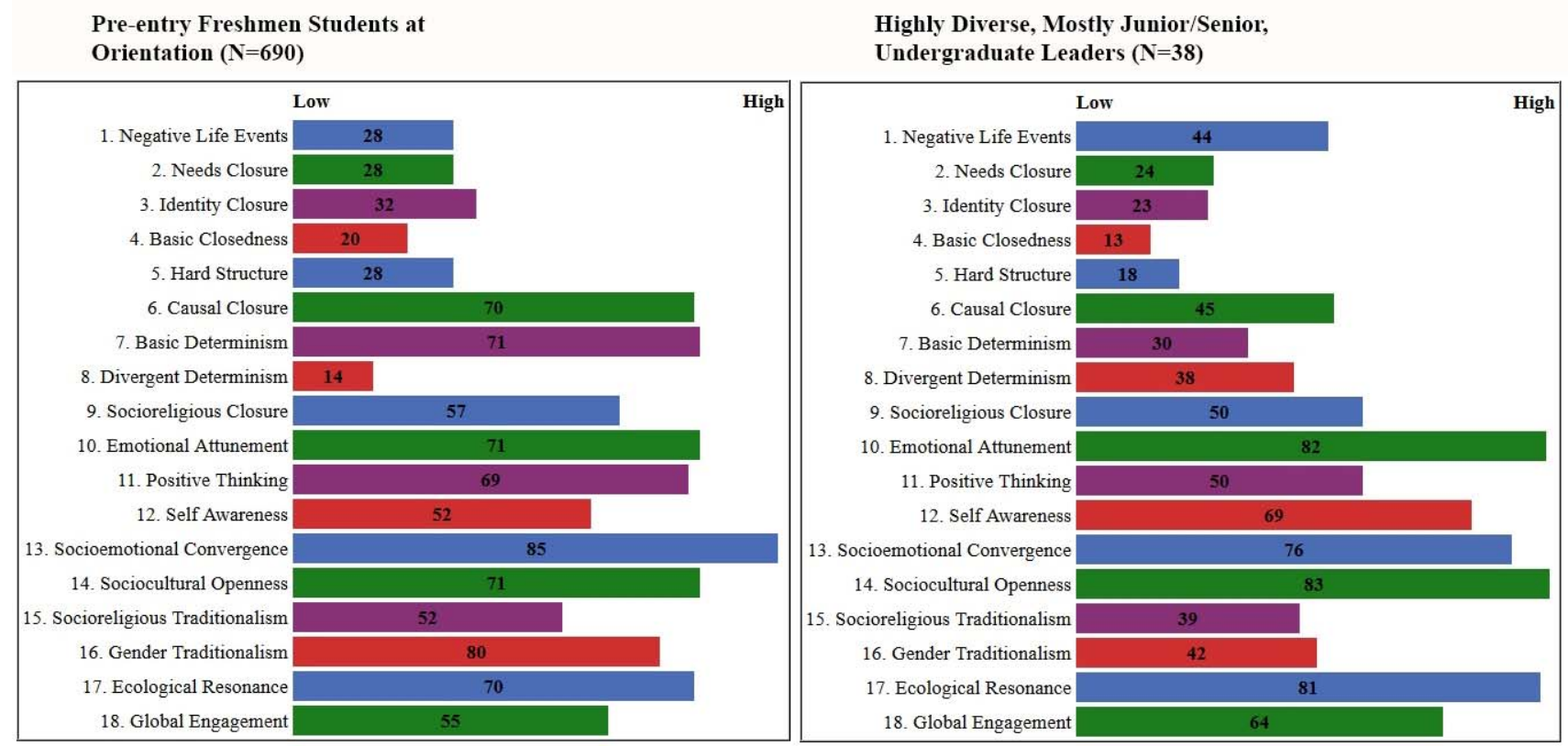

Figure 14. BEVI organizational profiles between two cohorts of students within the same institution. 
Another area of usage is to understand the similarities and differences between institutions. Consider for example Figure 15, which contrasts two BEVI profiles from two project partners: Dickinson College is a highly internationalized, majority female, liberal arts institution; Michigan Technological University (MTU) is not especially internationalized, is majority male, and has a strong engineering focus.
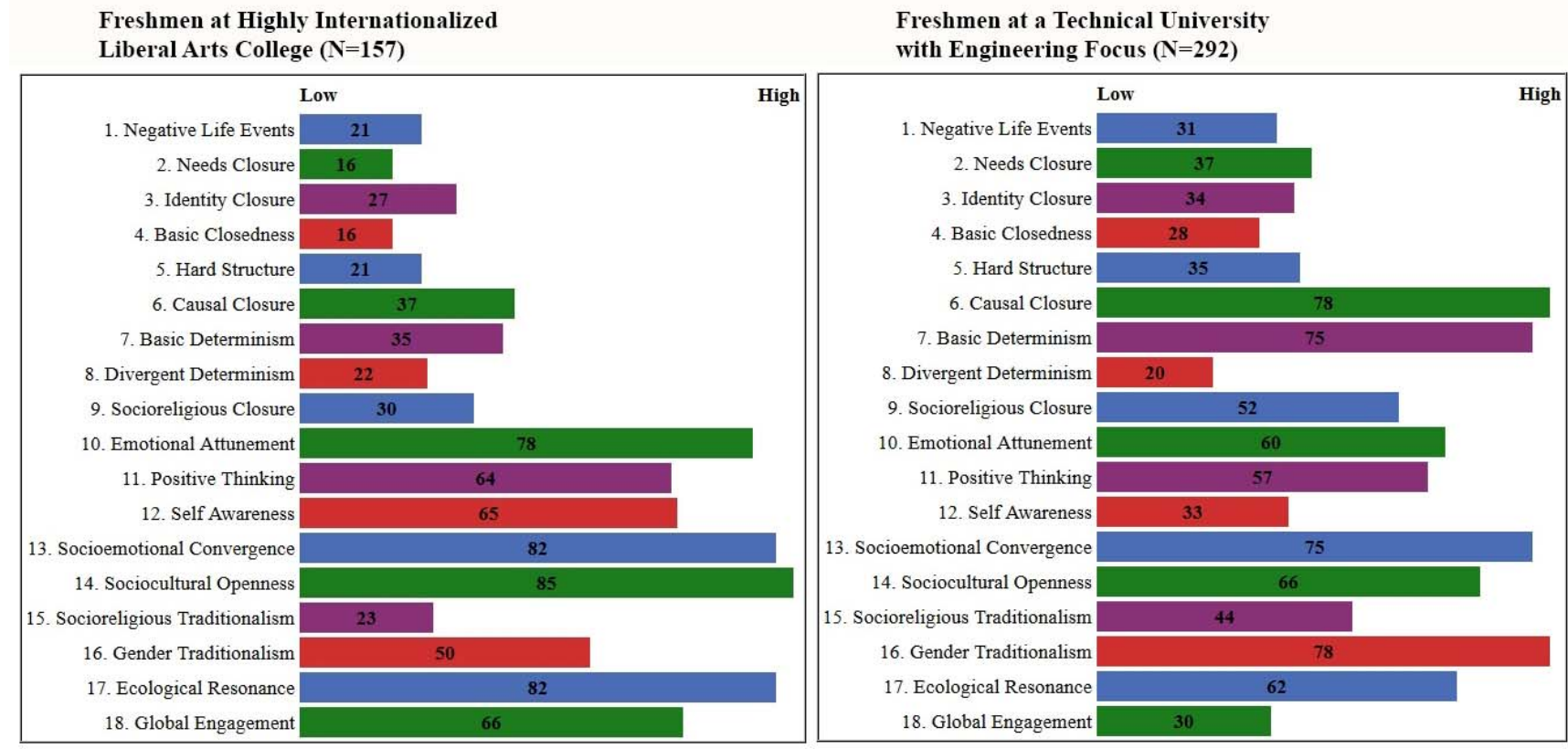

Figure 15. BEVI organizational profiles between two cohorts of students across different institutions. 
What do we conclude from these contrasting profiles? Among other notable distinctions, observe the differences on scales measuring the relative attributional complexity across the two institutions, as evidenced by percentile scores on Causal Closure (Dickinson $=37$ th percentile; $\mathrm{MTU}=78$ th percentile) and Basic Determinism (Dickinson $=35$ th percentile; $\mathrm{MTU}=75$ th percentile). From an interpretive standpoint, Dickinson "first years" from this sample at least evince a much greater tendency to recognize the complexity inherent in making attributions about self, others, and the larger world (i.e., they are much less likely to endorse black and white statements regarding why human beings are the way they are and/or why events happen as they do). To highlight a couple of other examples, Dickinson students from this sample are much more likely than MTU students to focus upon "inner" thoughts and experiences in self and other (Self Awareness: Dickinson $=65$ th percentile; $\mathrm{MTU}=33 \mathrm{rd}$ percentile) and much less likely to endorse traditional notions of what it means to be "female" or "male" (Gender Traditionalism: Dickinson = 50th percentile; $\mathrm{MTU}=78$ th percentile). Nothing in such a comparison is intended to illustrate or imply that one group of students is "better" than any other; the point is rather that we must understand our student bodies in order to appreciate where we are now (i.e., what our baseline is) and where we wish to go. Without such information, it is difficult to ensure that our instructional and administrative goals, methods, and plans are realistic, targeted, and consistent with overarching aspects of our student body and institutional mission.

Such assessment possibilities are aided further by another dimension of the reporting system, which distinguishes among "high," "medium," and "low" full scale scores across subgroups, as described above. Various indices and tables on the reporting system address this issue, including Background-Domain Contrast, which illustrates how different or similar the group is at the level of background information and domain scores by the lowest 30 percent, middle 40 percent, and highest 30 percent of full scale scores. Consider the following excerpts of Time 1/Time 2 Group Report results from a sample of participants $(\mathrm{N}=$ 101) on Background Domain Contrast.

Of other highlights that might be emphasized, note from Tables 11 and 12 that individuals who, at the outset of a learning experience, are at the low, middle, or high end of the "full scale" continuum demonstrate specific profile characteristics, both in terms of "background" variables (i.e., age, gender, education) and "domain" variables (i.e., one of the seven areas under which BEVI scales are clustered). In particular, for this sample of students, those most inclined toward an international, multicultural, or transformative learning experience are:

- $\quad$ older (average age $=27$ versus 19);

- approximately twice as likely to be female (ratio of females to males of 20:11 versus 16:15);

- more likely to be non-Caucasian than Caucasian (ratio of non-Caucasian to Caucasian of 11:20 versus 6:25);

- have more years of post-secondary education (7 years versus 2 years);

- more likely to come from a less wealthy family (\$70,000 versus $\$ 85,000)$; and,

- on the domain section of Background-Domain Contrast (comprised of specific BEVI scales), show substantial differences across the seven domains (e.g., 78 versus 32 on the domain of Global Access). 
Table 11

Sample Findings from Background Information Section of Background-Domain Contrast (N=101)

\begin{tabular}{|c|c|c|}
\hline Lowest Full Scale & Middle Full Scale & Highest Full Scale \\
\hline Mean $=41$ & Mean $=58$ & Mean $=71$ \\
\hline Background Information & Background Information & Background Information \\
\hline Average Age: 19 & Average Age: $\mathbf{2 2}$ & Average Age: 27 \\
\hline Males: 15 & Males: 11 & Males: 11 \\
\hline Females: 16 & Females: 26 & Females: $\mathbf{2 0}$ \\
\hline Caucasians: 25 & Caucasians: 26 & Caucasians: $\mathbf{2 0}$ \\
\hline Non-Caucasians: 6 & Non-Caucasians: $\mathbf{1 1}$ & Non-Caucasians: 11 \\
\hline Details: & Details: & Details: \\
\hline Raised in U.S.: $\mathbf{3 1}$ & Raised in U.S.: 37 & Raised in U.S.: 29 \\
\hline Not Raised in U.S.: 0 & Not Raised in U.S.: 0 & Not Raised in U.S.: $\mathbf{2}$ \\
\hline Details: & Details: & Details: \\
\hline Average Years of Education: 2 & Average Years of Education: 3 & Average Years of Education: 7 \\
\hline Average Parental / Household Income: $\$ 85,484$ & Average Parental / Household Income: \$77,162 & Average Parental / Household Income: \$70,161 \\
\hline
\end{tabular}


Table 12

Sample Findings from Domain Section of Background-Domain Contrast

\begin{tabular}{|c|c|c|}
\hline II. Full Scale: $\mathbf{4 1}$ & II. Full Scale: $\mathbf{5 8}$ & II. Full Scale: 71 \\
\hline $\begin{array}{l}\text { III. Formative Variables } \\
\begin{array}{l}\text { - Negative Life Events: } \mathbf{4 0} \\
\text { - See Background Information }\end{array}\end{array}$ & $\begin{array}{l}\text { III. Formative Variables } \\
\text { - Negative Life Events: } 64 \\
\text { - See Background Information }\end{array}$ & $\begin{array}{l}\text { III. Formative Variables } \\
\text { - Negative Life Events: } \mathbf{5 1} \\
\text { - See Background Information }\end{array}$ \\
\hline IV. Fulfillment of Core Needs: 37 & IV. Fulfillment of Core Needs: 69 & IV. Fulfillment of Core Needs: $\mathbf{8 2}$ \\
\hline V. Tolerance of Disequilibrium: 36 & V. Tolerance of Disequilibrium: $\mathbf{5 2}$ & V. Tolerance of Disequilibrium: 68 \\
\hline VI. Critical Thinking: 35 & VI. Critical Thinking: $\mathbf{5 1}$ & VI. Critical Thinking: 73 \\
\hline VII. Self Access: $\mathbf{5 7}$ & VII. Self Access: 76 & VII. Self Access: 78 \\
\hline VIII. Other Access: 30 & VIII. Other Access: 55 & VIII. Other Access: 78 \\
\hline IX: Global Access: 32 & IX: Global Access: 54 & IX: Global Access: 78 \\
\hline
\end{tabular}

From the standpoint of the report system, then, the implications of such findings should be clear. First, differences within groups should not be underestimated but expected (i.e., we should assume that groups may well have important differences both at the level of background and how they experience self, others, and the larger world). Second, for those who are leading learning, growth, or development experiences, reviewing such differences before a course, program, or workshop begins may be very helpful in understanding the nature of the group with which they will be interacting throughout the experience. Third, for appropriately trained and skilled facilitators, it may be helpful to share some or all results with participants in order to sensitize them to such differences and similarities among them (i.e., to highlight the fact that there may be very good reasons for why participants react as they do to the experiences they are about to have, as illustrated on several occasions through various projects reported in this article). Fourth and finally, such findings may help at the level of interpretation (i.e., the role of the instructor and/or learning experience should be accurately appraised in terms of relative impact) since, most likely, there will be multiple interactions occurring simultaneously that affect learning processes and outcomes. In short, by reviewing such reportbased results, more reflective, aware, and skillful learning interventions may be developed, implemented, and appraised since we have a basis for understanding why learning processes and outcomes unfold as they do.

Implication 13. By jointly utilizing individual and group reports, multiple opportunities for self- 
assessment, enhanced understanding, and group development may be facilitated. On many occasions, different cohorts have used the individual and group report system simultaneously in order to enhance understanding of how participants see themselves, others, and the larger world. By juxtaposing individual reports (which are personalized based upon unique scores, and read privately by each individual) with group reports (which show similarities and differences within the group, and are reviewed publicly with the group as a whole), abundant opportunities arise for dialogue, engagement, and growth.

Data from the Forum BEVI Project strongly suggest that the sorts of international, multicultural, and transformative experiences examined in this project $d o$ influence the amount and degree of growth, development, and learning that occur. But to illustrate such phenomena in real time to administrators, program directors, course/workshop instructors, students, and others who may have strong interests in such matters - but may not have a statistical background - various aspects of the report system are of particular relevance (i.e., to show in clear terms whether and how such "changes" actually occur). Because assessment results from various measures often are not communicated in a way that translates readily into real world usage, the Working Group devoted particular attention to how the BEVI's report system should be used from the standpoint of accessibility, relevance, and best practice. Consistent with our original charge, we were interested in understanding how the translation of data/scores into accessible report-based systems could be used to promote growth and learning in general, which should have implications for instrument-based report systems including, but not limited to, the BEVI. As such, considerable effort has been devoted to trying to understand how such a "report system" might best translate otherwise abstruse statistical findings into usable findings. In particular, considerable deliberation focused on how feedback from the individual reports of the BEVI should be given to students or staff. Among other conclusions, it was determined that a brief orientation by a trained BEVI administrator was deemed important in order to provide sufficient context for recipients. Moreover, given the comprehensive nature of BEVI reports, it proved important that those in leadership or administrative positions were sufficiently well-versed in matters of interpretation. That is because incomplete or superficial understanding of scales or indexes is contraindicated, especially when contemplating real world implications or applications based upon test scores or reports (e.g., Braskamp et al., 2012). In light of such considerations, it may be helpful to provide additional context prior to presenting actual sample reports that emerged during this project.

Essentially, the BEVI produces reports that may be used at three levels: individual, group, and organization. ${ }^{21}$ Individual reports are developed for each participant based upon their unique responses to the background information section of the BEVI along with their scores on specific BEVI scales. Depending upon their responses, a report is generated under the following headings, which correspond with the theoretical and empirical structure of the BEVI:

1. Introduction: Provides an overview of the BEVI.

2. The Foundation: 'Formative Variables' and 'Core Needs': Provides an indication of what the respondent reports about their own life history relative to others.

3. Tolerance of Disequilibrium: Describes whether the respondent sees him or herself as "very clear" or "not sure" about who they and others "are" under the auspices of how "confident" or "questioning" the respondent appears to be.

4. Making Sense of Why We Do What We Do: Indicates attributional tendencies in general (e.g., how and why people do what they do and why events happen as they do).

5. Access to Yourself and Your Thoughts, Feelings, and Needs: Describes how the individual deals with their own emotions as well as their interest in and predilection towards introspection and reflection upon "the self."

6. Access to the Thoughts and Feelings of Others: Describes how the person tends to regard

${ }^{21}$ This section and selected others in this article are adapted and/or excerpted from the BEVI test manual. (2015 The Forum on Education Abroad 
and experience issues that are of consequence at a sociocultural level (e.g., beliefs about politics, religion, gender, or the way society "should be structured").

7. Access to the Larger World: Indicates one's perspectives on "big picture" issues of the environment (e.g., the degree to which one is or is not concerned about ecological issues) and global engagement (e.g., the degree to which we should or should not be concerned about, or invested in, what is happening outside of our own country, culture, and context).

8. Conclusion: Provides context for the above report and offers closing thoughts to consider.

Individual reports are designed for application in a range of contexts and settings (e.g., as a teaching tool in classes; pre-departure orientation for an international learning experience; to promote growth and development in organizational and other applied contexts), and are intended to facilitate thoughtful and substantive reflection on self, others, and the world at large.

Group/Organizational reports. Basically, there are two types of group/organizational reports. First, Time 1 Group Reports describe results from the original administration of the BEVI to a specific group; secondly, Time 1/Time 2 Group Reports examine how groups change from one administration of the BEVI to the next (Time 1/Time 2/Time 3, etc. reports also may be derived, and are recommended in terms of tracking trends over the long term, as noted above). The primary difference between Time 1 and Time 1/Time 2 reports are a few indices that may be developed only on the basis of comparisons between groups from Time 1 to Time 2 (e.g., Worldview Shift). Each type of report includes a series of tables and indices that provide information about specific aspects of the group that completed the BEVI. For example, Background-Domain Contrast illustrates how different or similar the group is at the level of background information and domain scores by the lowest 30 percent, middle 40 percent, and highest 30 percent of full scale scores. As another example, Profile Contrast illustrates how different and similar the group is across all 17 BEVI scales via the lowest 30 percent, middle 40 percent, and highest 30 percent of full scale scores.

Mindful of the overarching goals of relevance and impact of such report-based assessment, including but not limited to the BEVI, questions that emerged consistently during our work with this measure (i.e., those that proved useful in facilitating interpretation and group-based discussion) included:

1. How would you describe the group, organization, or institution overall in terms of the most striking findings?

2. How is the group most different and most similar to itself?

3. Which findings are most surprising, and how can you make sense of such results on the basis of data that are provided?

4. Which findings were expected, and what do they suggest about your group, organization, or institution?

5. Which findings would be most relevant or interesting to a particular group or your overall organization/institution, and why?

6. How could findings inform or shape group-based discussions and processes?

7. What do findings suggest in terms of a particular course, program, or learning experience (e.g., Where might areas of consensus or conflict occur over time? How might patterns interact with a learning, growth, or development experience in terms of overarching goals?)?

8. What might findings suggest about the effectiveness of a particular learning, growth, or development experience?

9. How might findings be used to facilitate the development of new or improved learning, growth, or development experiences?

10. How might findings be used to track changes over time? 
There are many other questions to be asked of such report results. Overall, we learned that it was maximally important for users to take sufficient time to review and reflect upon reports in order to ascertain which tables, indices, or specific findings are most relevant for their specific goals and purposes.

Over the course of this project, the most common usage was a combination of group based and individual reports (i.e., during a pre-departure study abroad orientation program; at the outset of a multicultural course or workshop; in a residence hall at the beginning of the academic year). In this case, individual students would be offered the opportunity to receive their own individual report, which they (and only they) would read. Then, a group report that had been developed on the basis of the aggregate scores from this group of individuals would be shared with the group. In this way, individuals are able to reflect (in private) upon their own BEVI results with the indices and other scale information from the BEVI group report (in public). This dialectic process between private and public reflection - which allows for safe internal dialogue and voluntary external dialogue - seems to be integral to the sort of reflection, awareness, and insight that students and staff frequently reported from this process (e.g., "It helped to think about why I am who I am"; "It helps me appreciate why others believe what they do").

At another level, the Group Reports help program directors or course instructors understand better the differences and similarities of history or worldviews by the members of their group. For example, it was not uncommon for bimodal distributions on various scales (e.g., Gender Traditionalism; Socioreligious Traditionalism) to emerge, which when combined with the breakdown of scale scores by profile, permit the leader or administrator of a learning experience to understand better the makeup of a group even before departing and/or at the outset of a class. From an applied standpoint, such results may be used productively by showing group-based results and asking for volunteers to discuss "why they believe what they believe," a process that can lead to rich dialogue and reflection by participants (e.g., Iyer, 2013; Shealy, in press).

One common usage of the BEVI report system during this project was to engage students in an examination of the interface between their individual reports and the group report. To illustrate this process, we focus upon a learning community in one of our project partners, James Madison University, called Madison International, which brings together U.S. and international students as part of a living and learning community who complete coursework and participate in other experiences together during their first year at the university (see http://www.jmu.edu/international/isss/students/get-involved/mi/index.shtml). The example below of individual/group report usage focuses on a subset of these students $(\mathrm{N}=22)$, who completed a course called Making Sense of Beliefs and Values: A Guided Tour for Global Citizens. After providing consent and completing the BEVI, individual reports were provided to each student in the course. The first page of a sample individual report is shown in Figure 16.

The BEVI trained coordinator then reviews the Group Report with all members of the cohort. Depending on the goals of a particular BEVI workshop, different sections of the report may be emphasized. In the case of Madison International, most of the focus centered on three components of the Group Report: 1) Background Information (i.e., descriptive information regarding how the group "breaks down" across a range of different demographic variables); 2) Aggregate Profile (i.e., the aggregate scores for participants across all BEVI scales); and 3) Decile Profile (i.e., the aggregate scores across each scale in increments of 10 percent, in order to illustrate how the members of a group are dispersed across each of the BEVI scales). Figures 17 19 illustrate these three components of the BEVI Group Report for the Madison International Learning Community. 


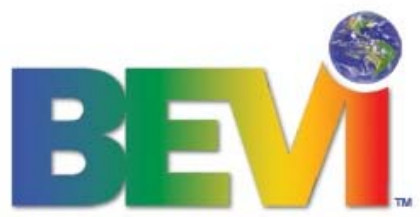

Beliefs, Events, and Values Inventory

\title{
You and Your Worldview
}

\author{
A Personal Report from the \\ Beliefs, Events, and Values Inventory (BEVI) ${ }^{T M}$
}

User: 9389488 Date of Test: 8/29/2012

The Beliefs, Events, and Values Inventory (BEVI) is designed to help people gain greater awareness of their own beliefs and values. The BEVI also examines how beliefs and values may influence learning, personal growth, relationships, group or organizational processes, and the pursuit of life goals. In addressing these issues, this report contains information about how you see yourself, others, and the larger world. However, because the BEVI takes no position at all on whether the answers you provided are right, wrong, true, or false, neither does this report make any such claims. Instead, this report is designed to promote reflection about issues in life that often are relevant to us all. If you have any questions about the BEVI, please contact the BEVI administrator at your college, university, or study abroad provider. You also may contact us directly at ibavi@ibavi.org. Before reading the following report, you must agree to the three conditions below either by clicking the Agree button or by signing a separate agreement form that will be provided to you prior to reading the report:

1. You (9389488) voluntarily completed the BEVI and subsequently requested this follow up report, which you are reading voluntarily (i.e., you agree that no person, group, organization, or entity is requiring you to read this report):

2. Every effort has been made to communicate information from the BEVI in a way that is helpful and constructive. However, if you experience distress upon reading this report, you agree to contact the BEVI administrator in your organization, the coordinator of a BEVI feedback session, and/or a health or mental health care provider.

3. You are free to keep a copy of this report for yourself. However, because the BEVI and this report are copyrighted, you agree not to photocopy this report or distribute it (electronically or otherwise) to anyone else.

\section{Introduction}

Pick up a newspaper or turn on a radio or television anywhere in the world and one fact becomes immediately clear: beliefs and values are integral to the human experience. People have strong opinions about topics from politics, religion, education and the arts, to marriage, family, gender, and sexuality. However, whether a specific belief is "right" or "wrong" is arguably less important than understanding the complex interaction among thoughts, feelings, behavior, life history, and context that results in

Figure 16. Sample introductory page from the BEVI individual report. 


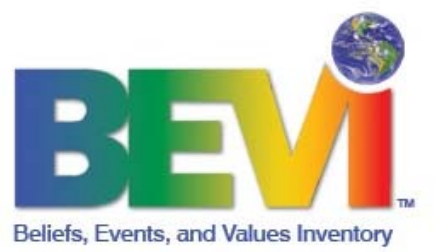

\section{BEVI Group Report}

\begin{tabular}{|c|c|c|}
\hline \multicolumn{3}{|l|}{ Madison International } \\
\hline \multicolumn{3}{|l|}{$\mathbf{N}=\mathbf{2 2}$} \\
\hline \multicolumn{3}{|l|}{ Background } \\
\hline \multicolumn{3}{|l|}{ Ethnic Group } \\
\hline Black And White & $4.55 \%$ & \\
\hline Black/African-American & $13.64 \%$ & \\
\hline Hispanic/Latino & $9.09 \%$ & \\
\hline Scandinavian & $4.55 \%$ & \\
\hline White & $68.18 \%$ & \\
\hline \multicolumn{3}{|l|}{ Gender } \\
\hline \multicolumn{3}{|l|}{ Female $63.64 \%$} \\
\hline Male $\quad 36.36 \%$ & & \\
\hline \multicolumn{3}{|l|}{ Political Orientation } \\
\hline Democrat & & $31.82 \%$ \\
\hline Independent & & $31.82 \%$ \\
\hline Philosophical Anarchist & & $4.55 \%$ \\
\hline Republican & & $27.27 \%$ \\
\hline Social Democrat (mostly & Democratic) & $4.55 \%$ \\
\hline
\end{tabular}

Figure 17. Introductory excerpt from the background information section of the group report for the Madison International Learning Community. 


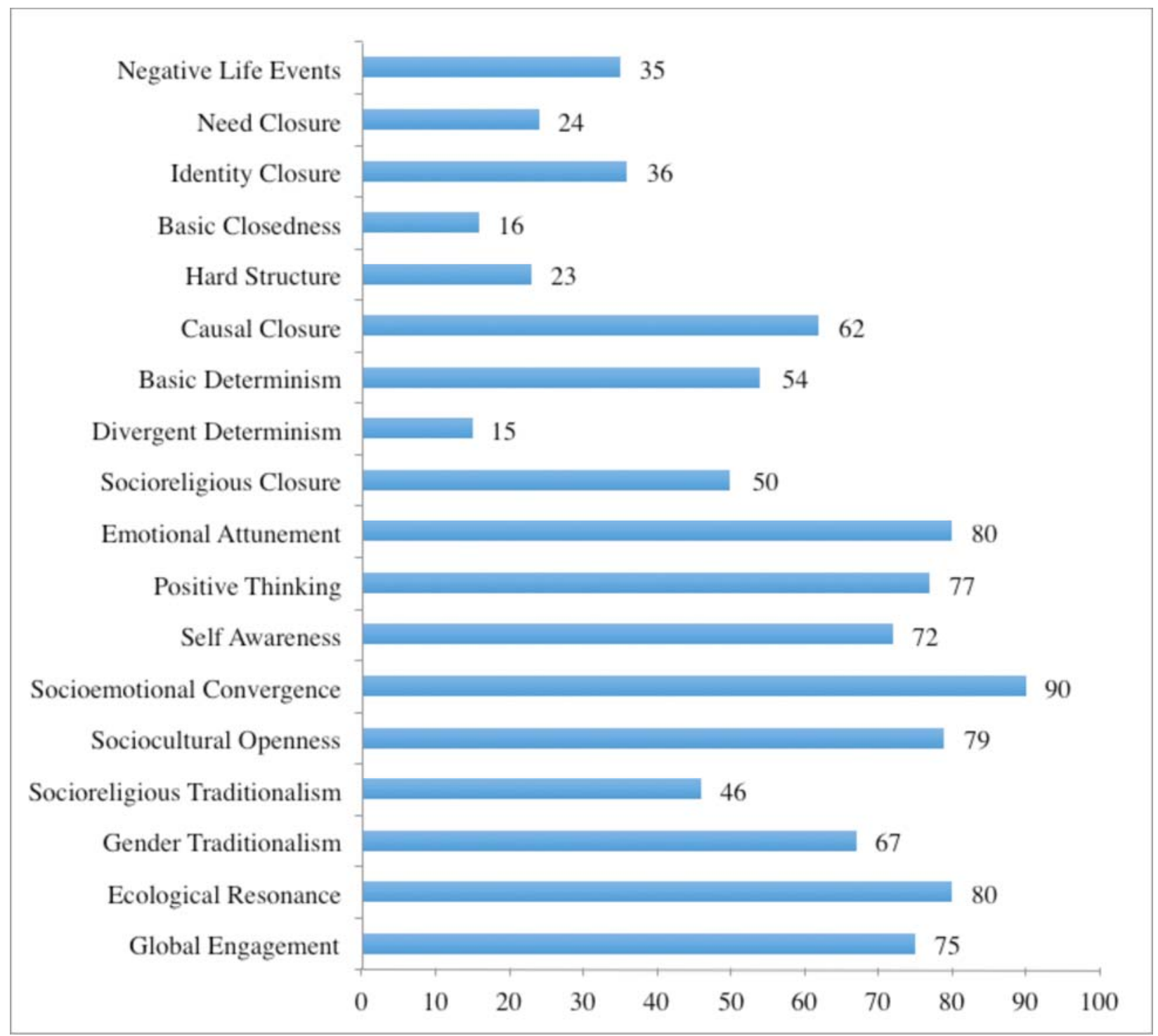

Figure 18. Aggregate Profile of the BEVI for the Madison International Learning Community. 


\begin{tabular}{|c|c|c|c|c|c|c|c|c|c|c|}
\hline Deciles: & 1 & 2 & 3 & 4 & 5 & 6 & 7 & 8 & 9 & 10 \\
\hline 1. Negative Life Events & $18 \%$ & $14 \%$ & $23 \%$ & $5 \%$ & $9 \%$ & $0 \%$ & $14 \%$ & $9 \%$ & $5 \%$ & $5 \%$ \\
\hline 2. Needs Closure & $23 \%$ & $14 \%$ & $23 \%$ & $18 \%$ & $23 \%$ & $0 \%$ & $0 \%$ & $0 \%$ & $0 \%$ & $0 \%$ \\
\hline 3. Identity Closure & $23 \%$ & $5 \%$ & $5 \%$ & $23 \%$ & $36 \%$ & $5 \%$ & $0 \%$ & $0 \%$ & $0 \%$ & $5 \%$ \\
\hline 4. Basic Closedness & $45 \%$ & $9 \%$ & $5 \%$ & $23 \%$ & $14 \%$ & $5 \%$ & $0 \%$ & $0 \%$ & $0 \%$ & $0 \%$ \\
\hline 5. Hard Structure & $32 \%$ & $18 \%$ & $5 \%$ & $9 \%$ & $18 \%$ & $0 \%$ & $9 \%$ & $5 \%$ & $5 \%$ & $0 \%$ \\
\hline 6. Causal Closure & $9 \%$ & $0 \%$ & $18 \%$ & $0 \%$ & $5 \%$ & $27 \%$ & $14 \%$ & $0 \%$ & $9 \%$ & $18 \%$ \\
\hline 7. Basic Determinism & $5 \%$ & $9 \%$ & $18 \%$ & $14 \%$ & $9 \%$ & $9 \%$ & $18 \%$ & $0 \%$ & $5 \%$ & $14 \%$ \\
\hline 8. Divergent Determinism & $32 \%$ & $18 \%$ & $32 \%$ & $5 \%$ & $5 \%$ & $5 \%$ & $0 \%$ & $0 \%$ & $0 \%$ & $5 \%$ \\
\hline 9. Socioreligious Closure & $18 \%$ & $5 \%$ & $9 \%$ & $5 \%$ & $5 \%$ & $18 \%$ & $5 \%$ & $9 \%$ & $23 \%$ & $5 \%$ \\
\hline 10. Emotional Attunement & $0 \%$ & $0 \%$ & $5 \%$ & $0 \%$ & $5 \%$ & $14 \%$ & $5 \%$ & $18 \%$ & $27 \%$ & $27 \%$ \\
\hline 11. Positive Thinking & $5 \%$ & $0 \%$ & $9 \%$ & $9 \%$ & $9 \%$ & $9 \%$ & $9 \%$ & $9 \%$ & $14 \%$ & $27 \%$ \\
\hline 12. Self Awareness & $14 \%$ & $9 \%$ & $0 \%$ & $9 \%$ & $5 \%$ & $5 \%$ & $0 \%$ & $9 \%$ & $14 \%$ & $36 \%$ \\
\hline 13. Socioemotional Convergence & $0 \%$ & $0 \%$ & $0 \%$ & $0 \%$ & $0 \%$ & $5 \%$ & $9 \%$ & $18 \%$ & $27 \%$ & $41 \%$ \\
\hline 14. Sociocultural Openness & $0 \%$ & $0 \%$ & $0 \%$ & $0 \%$ & $0 \%$ & $14 \%$ & $14 \%$ & $23 \%$ & $32 \%$ & $18 \%$ \\
\hline 15. Socioreligious Traditionalism & $23 \%$ & $5 \%$ & $9 \%$ & $14 \%$ & $0 \%$ & $0 \%$ & $0 \%$ & $32 \%$ & $9 \%$ & $9 \%$ \\
\hline 16. Gender Traditionalism & $5 \%$ & $5 \%$ & $9 \%$ & $0 \%$ & $14 \%$ & $9 \%$ & $9 \%$ & $14 \%$ & $23 \%$ & $14 \%$ \\
\hline 17. Ecological Resonance & $0 \%$ & $0 \%$ & $0 \%$ & $0 \%$ & $5 \%$ & $27 \%$ & $9 \%$ & $14 \%$ & $14 \%$ & $32 \%$ \\
\hline 18. Global Engagement & $5 \%$ & $9 \%$ & $0 \%$ & $14 \%$ & $5 \%$ & $5 \%$ & $14 \%$ & $9 \%$ & $18 \%$ & $23 \%$ \\
\hline Deciles: & 1 & 2 & 3 & 4 & 5 & 6 & 7 & 8 & 9 & 10 \\
\hline
\end{tabular}

Figure 19. Decile Profile of the BEVI for the Madison International Learning Community.

How were the above three components of the BEVI group profile used with the Madison International Learning Community? After reviewing the background characteristics of the group (e.g., highlighting areas of similarity and difference, from Figure 17), the bulk of this session focused on explaining and discussing the results from the Aggregate Profile and Decile Profile. To highlight a number of these scales, and among other findings that might be emphasized, note overall that the group as a whole (from the Aggregate Profile in Figure 18):

- reported relatively positive life histories (e.g., low Negative Life Events, Needs Closure);

- is quite open to basic thoughts/feelings that characterize the typical experience for most human beings (e.g., low Basic Closedness);

- demonstrates a moderate degree of attributional complexity regarding why human beings do what they do and why events in the world happen as they do (e.g., moderate Basic

Determinism);

- $\quad$ evidences a moderate degree of religiosity (e.g., moderate Socioreligious Traditionalism);

- $\quad$ is highly self and emotionally aware (e.g., high Emotional Attunement, Self Awareness);

- appears to possess a high capacity to experience self and others in shades of gray rather than in dichotomous terms (e.g., high Socioemotional Convergence);

- is very open to cultural beliefs and practices that are different from their own (e.g., high Sociocultural Openness);

- is concerned about the environment and natural world (e.g., high Ecological Resonance); and,

- is deeply interested in making a difference in the world (e.g., high Global Engagement). 
In many ways, such a profile would perhaps be consistent with a group of individuals who have selfselected to be part of an international living and learning community. However, as noted throughout this article, it is very important to go beyond aggregated results in order to understand areas of difference and similarity within the group as a whole. To do so, let's focus on one of the BEVI scales, Socioreligious Traditionalism, from the Decile Profile (Figure 19), particularly because this scale promoted a great deal of reflection and discussion by community members. As illustrated below in Figure 20, note first the extraordinary dispersion by the group across this specific scale from Decile Profile.

\begin{tabular}{|l|l|l|l|l|l|l|l|l|l|l|}
\hline 15. Socioreligious Traditionalism & $23 \%$ & $5 \%$ & $9 \%$ & $14 \%$ & $0 \%$ & $0 \%$ & $0 \%$ & $32 \%$ & $9 \%$ & $9 \%$ \\
\hline
\end{tabular}

Figure 20. The Socioreligious Traditionalism scale from the Decile Profile for the Madison International Learning Community.

In particular, approximately 50 percent of the learning community falls between the $1^{\text {st }}$ and $40^{\text {th }}$ percentile of the scale, whereas the remaining 50 percent falls between the $70^{\text {th }}$ and $100^{\text {th }}$ percentile (no respondents fall between the $41^{\text {st }}$ and $69^{\text {th }}$ percentile). Note further that approximately one quarter of the community occupies the lowest $10^{\text {th }}$ percentile on this scale (i.e., is essentially disavowing religion and religious belief). The presentation of this finding sparked a great deal of dialogue by the group. From a thematic perspective, the group seemed fascinated by the fact that such profound differences existed among individual members. They also discussed larger issues, such as the purpose of existence, where beliefs and values come from, and why such questions should - or should not - matter to us as individuals and as a society. In short, the juxtaposition of individual and group reports offers a model for how the individual learner may have a private encounter with "self" regarding one's own worldview, to be followed by a skillfully facilitated dialogue on how the larger group sees self, others, and the larger world. Such a process may well promote a great deal of learning, growth, and development in a manner that is consistent with larger course, program, and institutional goals (Iyer, 2013).

Implication 14. Changes in beliefs and values about self, others, and the world at large appear determined largely by the $7 D$ s (duration, difference, depth, determine, design, deliver, debrief). The odds of facilitating effective learning, development, and growth opportunities are enhanced considerably by attending to the $7 D$ s before, during, and after a learning intervention is developed, implemented, evaluated, and refined.

As noted throughout this article, to understand and assess the processes and outcomes of international, multicultural, and transformative learning, it is crucial both to measure and account for the impact of withingroup differences on the learning processes and outcomes we observe.

Specifically, the "7Ds" (Shealy, in press) include the following variables:

1. duration (i.e., how long an international, multicultural, or transformative education experience occurs);

2. difference (i.e., how different the experience is from what the "self" of the experiencer is accustomed);

3. depth (i.e., what the capacity of the learner is to experience all that the intervention is able to convey);

4. determine (i.e., through formal and informal assessment, how well the intervener understands his/her audience);

5. design (i.e., based upon knowledge of the audience and careful deliberation and development, what the quality of the intervention is);

6. deliver (i.e., how well the intervener can fulfill the transformative potential of the intervention); and, 
7. debrief (i.e., before, during, and after the intervention, how deeply the intervener assesses the nature of the learning experience, and uses such feedback to improve planned, current, and future interventions).

Although the 7Ds are based upon a combination of data-driven findings and best practice recommendations, future research could examine specific aspects of each in greater detail. For example, it would be possible to evaluate empirically how a relative understanding of one's audience is associated both with various characteristics of the audience (e.g., from BEVI group scores) as well as differences in learning outcomes (e.g., to what degree congruency between the intervener and the audience is consistent with better learning outcomes, such as higher grades or greater satisfaction). In short, the $7 \mathrm{Ds}$ are offered as data/practice based hypotheses that may help in the generation of specific questions that are worthy of further investigation.

Implication 15. Best practice in assessment requires best practice in research. By attending to data-based findings regarding the mediators and moderators of teaching, training, and learning - and adopting mixed methods approaches to assessment that account for the complexity of the variables at hand - we are much better able to plan for, implement, and evaluate the effectiveness of our interventions. Ultimately, our goals are to: 1) understand the complex interactions between teachers, trainers, and learners as well as the complex processes and outcomes of learning, growth, and development, and 2) use such information to enhance the quality and impact of teaching, training, and learning in the real world.

This fifteenth and final implication really is a take-home message from the entire Forum BEVI Project. As envisioned in the original charge to the Working Group, we knew that our ability to hone in on very complex and interacting processes of learning, growth, and development would require exquisite attention to myriad issues over a sustained period of time. Several of these "lessons learned" were codified in a symposium delivered at the Association for International Education Administrators (AIEA) in the fourth year of this project (Gerber, Gottlieb, Pysarchik, \& Shealy, 2011), which sought to summarize best practice recommendations from four big picture assessment initiatives, including the Forum BEVI Project. These include the following six recommendations:

1. Assess Deeply (e.g., encourage innovation; integrate mixed methods; strive for ecological validity; evaluate interactions, mediators, and levels of analysis);

2. Address Challenges (e.g., political, time, economic; science/humanities split; not knowing what one doesn't know);

3. Secure Support (e.g., institutional/organizational; develop functional team; identify and pool resources);

4. Ensure Expertise (e.g., theory/data link; research design; psychometrics; statistical);

5. Develop Plan (e.g., specify assessment goals and outcomes; select measures; assign roles and responsibilities; monitor/correct processes); and

6. Value Collaboration (e.g., recognize team talents; appraise/meet local needs; facilitate buy in; solicit input/feedback).

From our perspective, these six recommendations provide a sense of what is necessary to design, implement, and evaluate a multi-site, multi-year assessment project in a manner that is more likely than not to generate outcomes of substance and impact. More than anything, beyond the letter of such recommendations, their spirit requires the cultivation of patience, kindness, and humor. That is because the one constant we encountered throughout was change - at the level of direction, approach, method, interpretation, partners, technique, administration, analyses, logistics, and so forth. Thus, there can be no substitute for relying on relational connections and good will toward one another, established through countless phone calls, emails, meetings, and presentations, over many years of collaborative work together. 


\section{Forum BEVI Project: Summary and Conclusion}

We began this article by offering a big picture perspective regarding the what and why of internationalization within the field of higher education. We turned then to an overview of contemporary efforts in the assessment of international, multicultural, and transformative learning. We next observed that the complex variables that mediate and moderate global learning outcomes still await precise identification. In particular, it behooves scholars, educators, and administrators to recognize and account for the profound and underlying human complexities - affective, behavioral, cognitive, contextual, cultural, developmental, historical - that interact with a particular international, multicultural, and transformative learning experience to produce the processes and outcomes that we ultimately observe. We then described the partnership formed in 2007 between the Forum on Education Abroad and International Beliefs and Values Institute, summarizing the goals and activities of the Forum BEVI Project Working Group. We provided a brief overview of the Beliefs, Events, and Values Inventory and its Equilintegration or EI theoretical framework. We presented specific applications of the BEVI throughout this initiative, and then provided an overview of the analyses and sample characteristics that informed our work. Finally, we discussed the 15 implications in detail.

We recognize fully that the above discussion is, to say the least, comprehensive and intensive, which befits a six year initiative involving multiple institutions and organizations as well as hundreds of analyses and thousands of participants. Ultimately, it is our hope that this project and its fifteen implications contribute to 1) a deeper understanding of the complex interactions and fascinating processes that are associated with who learns what and why, and under what circumstances as well as 2) greater appreciation for the demonstrable fact that such complexities can, and must, be assessed with depth, persistence, and rigor. In this regard, throughout our work, it has been heartening to experience our collective sophistication grow - theoretically, methodologically, empirically, and in applied terms - with attendant and constructive impacts upon a range of programs, courses, activities, and processes in the real world. From our perspective, the sine qua non of such work has been realized in the "aha" moments we all have shared. Formerly abstruse dynamics suddenly have been illuminated when we observe, for example, that conflicts or issues in a group actually are revealed via a bimodal distribution on specific scales, which indicate vast differences - or remarkable similarities - among cohort members in how and why they experience self, others, and the larger world as they do, with concomitant implications for group relations and learning processes over the short- and long-term. Likewise, we no longer get many questions like, "Why are you asking about life events?" as we have shown and learned together that the perception of one's own life history may change, often substantially, following exposure to cultures and contexts that are significantly different from one's own. In short, it seems increasingly to be understood - on the basis of theoretical framing, empirical data, and real world interventions - that who we are prior to a learning experience must be taken into account if we are to understand how and why the "7Ds" interact to produce the learning processes or outcomes that we do or do not observe, including (but not limited to) 1) the learning experiences we create and deliver; 2) what people bring to the learning experience based upon their life histories and worldviews; 3) how we're built and what we need as human beings; and 4) the ubiquitous interactions among these and related levels of analysis. Such findings seem well illuminated by the EI framework - that we inexorably are built to create a "version of reality" in the service of our core needs, the formative variables that shape and define us, and the subsequent beliefs and values that we call our own which further influence how and why we experience self, others, and the larger world as we do (e.g., Shealy, in press; Shealy et al., 2012).

As scholars, educators, professional staff, and administrators, we now have a better basis for understanding how our work interacts with the lives and worldviews of those who come before us seeking to learn, grow, and change, even if they - and we - may not apprehend yet what the implications are for them, and us, of engaging in these immersion processes so closely together. The fact that we co-create meaning through our shared lives and experiences is a substantive conclusion of this project that warrants further 
exploration, as we seek to hone in that much more on the interactions that ultimately are associated with who learns what and why, and under what circumstances (e.g., Cultivating the Globally Sustainable Self, 2015). In short, we must take our responsibilities very seriously, not only because that's "the right thing to do," but because abundant evidence from this project suggests that we may differ substantially in our capacity and inclination to carry out these important obligations as facilitators of learning, growth, and development. From our perspective, such an empirical reality is not, and should not be, an impetus for anxiety or doubt, but rather an opportunity to engage in a deeper exploration of how we may better fulfill our potential as educators, program developers, administrators, and scholars. Just as we expect our students to learn and grow, findings from this project indicate that we can and should expect no less of ourselves, even as we pursue these means and ends with a steadfast appreciation that we never are "done" vis-à-vis our own personal and professional development.

In the final analysis, what has been most gratifying for all of us over the years is to hear so often that what we have been trying to understand and explicate through this initiative has in fact been impactful, meaningful, and relevant. Through our work, we have come to see more clearly that we are - ineluctably change agents who are granted the privilege of transforming the learners who enter our educational institutions and organizations, and are in turn, transformed ourselves. Since we are charged to be agents of change by the next generation of citizens - and compelled by the pressing needs and opportunities of our increasingly interdependent world - it behooves us to answer this call in a manner that is optimally informed and maximally effective. Hopefully, the implications emerging from this project, combined with ongoing collaborative research and practice in multiple local and global settings, ${ }^{22}$ will help us all assess international, multicultural, and transformative learning - and apply our insights and understandings - with care, capacity, and commitment in the months and years ahead.

\section{References}

American Council on the Teaching of Foreign Languages (2012). Proficiency guidelines. Retrieved from http://www.actfl.org/files/public/ACTFLProficiency Guidelines2012_FINAL.pdf.

Arum, R. \& Roksa, J. (2011). Academically adrift: Limited learning on college campuses. Chicago, IL: University of Chicago Press.

Association of American Colleges and Universities (2007). College learning for the new global century: A report from the National Leadership Council for Liberal Education and America's Promise. Washington, DC: Peter D. Hart Research Associates. Retrieved from www.aacu.org/leap/documents/GlobalCentury final.pdf.

Association of Commonwealth Universities' Management Benchmarking Program (2012). Retrieved from https://www.acu.ac.uk/focus-areas/research-management-uptake/research-management-benchmarkingprogramme/.

Atwood, K. Chkhaidze, N., Shealy, C. N., Staton, R., Sternberger, L. G. (2014, August). Transformation of self: Implications from the Forum BEVI Project. Poster presented at the annual meeting of the American Psychological Association, Washington, DC.

Beliefs, Events, and Values Inventory. Retrieved from http://www.thebevi.com/.

Bennett, M. J. (1986). A developmental approach to training intercultural sensitivity. In J. Martin (Guest

${ }^{22}$ Allied scholarship is examined further in Cultivating the Globally Sustainable Self, a three year summit series on transformative teaching, training, and learning in research and practice, which began in March of 2015

(see http://www.jmu.edu/summitseries). 
Ed.), Special issue on intercultural training, International Journal of Intercultural Relations, 10(2), 179186.

Bennett, M. J. (1993). Towards ethnorelativism: A developmental model of intercultural sensitivity (revised). In R. M. Paige (Ed.), Education for the intercultural experience (pp. 21-71). Yarmouth, ME: Intercultural Press.

Bennett, M. J., \& Hammer, M. R. (2002). The Intercultural Development Inventory. Portland, OR: The IDI Corporation.

Bhawuk, D. P. S., \& Brislin, R. (1992). The measurement of intercultural sensitivity using the concepts of individualism and collectivism. International Journal of Intercultural Relations, 16(4), 413-436.

Blair, S., Latz, G., Sternberger, L.G., \& Shealy, C.N. (February, 2014). Assessing global learning: Leadership practices and imperatives for the 21 st century academy. Workshop presented at the annual conference of the Association of International Education Administrators, Washington, D.C.

Braskamp, L. A., Braskamp, D. C., Merrill, K. C., \& Engberg, M. E. (2010). Global Perspective Inventory (GPI). Retrieved from https://gpi.central.edu.

Braskamp, L. A., Blair, S., Shealy, C. N., \& Hammer, M. R. (2012). Which tool does what? Aligning assessment tools to Forum standards. Retrieved from http://apps.forumea.org/documents/Forum-Denver-2012-WhichToolDoesWhat--Introduction--S.Blair.pdf.

Braskamp, L. A. \& Engberg, M. E. (2014). Guidelines for judging the effectiveness of assessing student learning. Chicago, IL: University of Chicago Press.

Brearly, T., Shealy, C. N., Staton, R., \& Sternberger, L. G. (2012, August). Prejudice and religious and nonreligious certitude: An empirical analysis from the Forum BEVI Project. Poster presented at the annual meeting of the American Psychological Association, Orlando, FL.

Chang, W., Yuan, Y., \& Chuang, Y. (2013). The relationship between international experience and crosscultural adaptability. International Journal of Intercultural Relations, 37(2), 268-273.

Chen, G. M. \& Starosta, W. J. (2000a). The development and validation of the international communication sensitivity scale. Human Communication, 3(1), 2-14.

Chen, G. M. \& Starosta, W. J. (2000b). Intercultural sensitivity. In L. A. Samovar \& R. E. Porter (Eds.), Intercultural communication: A reader (pp. 406-413). Belmont, CA: Wadsworth Publishing Company.

Church, A. T. (1982). Sojourner adjustment. Psychological Bulletin 91(3), 540-572.

Clarke, I., Flaherty, T., Wright, N. D., \& McMillen, R. M. (2009). Student intercultural proficiency from study abroad programs. Journal of Marketing Education, 31(2), 173-181.

Coates, M., Hanson, W., Samuel, D.B., Ashe, M., \& Cozen, J. The Beliefs, Events, and Values Inventory (in press). Psychological assessment implications and applications. In C. N. Shealy (Ed.), Making sense of beliefs and values. New York, NY: Springer Publishing.

Corbitt, J. N. (2011). Global awareness. Retrieved from http://www.globalawarenessprofile.com/blog/.

Coryell, J. E., Durodoye, B. A., Wright, R. R., Pate, P. E., \& Nguyen, S. (2012). Case studies of internationalization in adult and higher education: Inside the processes of four universities in the United 
States and the United Kingdom. Journal of Studies in International Education, 16(1), 75-98.

Cottrell, S. (2005) Critical thinking skills. Palgrave, USA.

Cozen, J., Hanson, W., Poston, J., Jones, S., \& Tabit, M. (in press). The Beliefs, Events, and Values Inventory (BEVI): Implications and applications for therapeutic assessment and intervention. In C. N. Shealy (Ed.), Making sense of beliefs and values. New York, NY: Springer Publishing.

Creswell, J. W., \& Plano-Clark, V. L. (2007). Designing and conducting mixed methods research. Thousand Oaks, CA: SAGE Publications, Inc.

Cultivating the Globally Sustainable Self (2015). Retrieved from www.jmu.edu/summitseries.

Deardorff, D. K (2006). Identification and assessment of intercultural competence as a student outcome of internationalization. Journal of Studies in International Education, 10(3), 241-266.

Deardorff, D. K. \& Van Gaalen, A. (2012). Outcomes assessment in the internationalization of higher education. In D. K. Deardorff, H. de Wit, J. D. Heyl, \& T. Adams (Eds.), The SAGE handbook of international higher education (pp. 167-189). Thousand Oaks, CA: SAGE Publications, Inc.

Der-Karabetian, A. (1992). World-mindedness and the nuclear threat: A multinational study. Journal of Social Behavior and Personality, 7(2), 293-308.

Der-Karabetian, A., \& Metzer, J. (1993). The Cross-cultural World-mindedness Scale and political party affiliation. Psychological Reports, 72(3), 1069-1070.

De Wit, H., \& Merkx, G. (2012). The history of internationalization of higher education. In D. K. Deardorff, H. de Wit, J. D. Heyl, \& T. Adams (Eds.), The SAGE handbook of international higher education (pp. 43-57). Thousand Oaks, CA: SAGE Publications, Inc.

Doyle, D. (Fall 2009). Holistic assessment and the study abroad experience. Frontiers: The Interdisciplinary Journal of Study Abroad, XVIII, 143-156.

Duru, E., \& Poyrazli, S. (2007). Personality dimensions, psychosocial-demographic variables, and English language competency in predicting level of acculturative stress among Turkish international students. International Journal of Stress Management, 14(1), 99-110.

Ellis, I. E., Lee, J. M., \& Wiley, L. P. (2009). Educator dispositions: A survey of U.S. teacher education programs. Southeastern Teacher Education Journal, 2(3), 77-86.

Ennis, R. (Winter 1962). A concept of critical thinking. Harvard Educational Review, 32(1), 81-111.

Ethics of International Engagement and Service-Learning Project (2011). Retrieved from http://ethicsofisl.ubc.ca.

Fantini, A.E. (2000). A central concern: Developing intercultural competence. Retrieved from http://www.adam-europe.eu/prj/2935/prd/8/1/develop-I-com.pdf.

Fantini, A.E. (2006). Assessment tools of intercultural communicative competence. Retrieved from http://federationeil.org/documents/AppendixF.pdf.

Fantini, A.E. (2009). Assessing intercultural competence. In D. K. Deardorff (Ed.), The SAGE handbook of intercultural competence (pp. 456-476). Thousand Oaks, CA: SAGE Publications, Inc. 
Fisher, A. \& Scriven, M. (1997). Critical thinking. Its definition and assessment. Inverness, CA: Edgepress.

Forum - IBAVI Working Group on BEVI Implementation (2012, March). The who, what, and why of international, multicultural, and transformative learning: Recent findings from the Forum BEVI Project. Paper presented at the $8^{\text {th }}$ annual conference of the Forum on Education Abroad, Denver, CO.

Forum on Education Abroad. (2005). A baseline survey of curriculum integration in education abroad. Northampton, MA.

Forum on Education Abroad. (2007). A guide to outcomes assessment in education abroad. Retrieved from https://www.forumea.org/resources/outcomes/guide.

Forum-BEVI Project. (2015). Retrieved from http://www.forumea.org/research-bevi-project.cfm.

Geisinger, K. F., (Ed.) (2013). APA handbook of testing and assessment in psychology, Vol.1. Washington, DC: American Psychological Association.

Gerber, T. P., Gottlieb, E. E., Pysarchik, D. T., \& Shealy, C. N. (2011). International program evaluation: The power of collaboration. Symposium presented at the annual meeting of the Association of International Education Administrators, San Francisco, CA.

Green, M. (2013 Nov/Dec). Acting as global citizens: A challenge to U.S. colleges and universities. International Educator (22)6, 52-55.

Halpern, D.F. (1998). Teaching critical thinking for transfer across domains. Dispositions, skills, structure training, and metacognitive monitoring. American Psychologist, 53(4), 449-455.

Hammer, M. R., Bennett, M. J, and Wiseman, R. (2003). Measuring intercultural sensitivity: The Intercultural Development Inventory. International Journal of Intercultural Relations, 27(4), 421-443.

Hammer, M. R. (2012). The Intercultural Development Inventory (IDI): Making intercultural competence development a reality. Paper presented at the 8th annual conference of the Forum on Education Abroad, Denver, CO.

Hanson, W. E., Leighton, J. P., Donaldson, S. I., Terjesen, M., \& Shealy, C. N. (in press). Assessment: From psychological testing and program evaluation to feedback, intervention, and positive worldwide change. In C. N. Shealy, \& M. Bullock (Eds.), Going global: How psychology and psychologists can meet a world of need. Washington, DC: American Psychological Association.

Harkness, J. (2003). Questionnaire translation. In J. Harkness, F. Van de Vijer, \& P. Moher (Eds.), Crosscultural survey methods (pp. 35-56). Hoboken, NJ: John Wiley \& Sons, Inc.

Hayes, D. J., Shealy, C. N., Sivo, S. A., \& Weinstein, Z. C. (1999). Psychology, religion, and scale 5 (religious traditionalism) of the "BEVI". Poster session presented at the meeting of the American Psychological Association, Boston, MA.

Hett, E. J. (1993). The development of an instrument to measure global-mindedness (Doctoral dissertation). University of San Diego, San Diego, CA.

Hill, C., Johnson, M., Brinton, C., Staton, R., Sternberger, L., \& Shealy (2013, August). The etiology of economic beliefs and values: Implications and applications from the Forum BEVI Project. Poster presented at the annual meeting of the American Psychological Association, Honolulu, HI. 
Hu, C., Pazaki, H., \& Velander, E. (2014). Evaluating global education at a regional university: A focus group research on faculty perspectives. Theory in Action (7)1, 65-78.

Inglehart, R., Basánez, M., Díez-Medrano, J., Halman, L., \& Luijkx, R. (2004). Human beliefs and values: A cross-cultural sourcebook based on the 1999-2002 values surveys. Mexico: Siglo XXI Editores.

Inglehart, R., Basáńez M., \& Moreno, A. (1998). Human values and beliefs: A cross-cultural sourcebook. Ann Arbor, MI: The University of Michigan Press.

Institute of International Education. (2014). Open Doors report. Retrieved from http://www.iie.org/Research-and-Publications/Open-Doors/Data.

Interstate New Teacher Assessment and Support Consortium (InTASC). (2011). Model core teaching standards: A resource for state dialogue. Retrieved from http://www.ccsso.org/documents/2011/intasc model core teaching standards 2011.pdf.

Interagency Language Roundtable. (2011). ILR proficiency levels. Retrieved from http://www.govtilr.org/skills/ILRscale1.htm.

International Association of Universities (2010). Internationalization of higher education: Global trends, regional perspectives. IAU $3^{\text {rd }}$ Global Survey Report. Paris, France: International Association of Universities.

International Beliefs and Values Institute. Retrieved from http://www.ibavi.org/.

Isley, E. B., Shealy, C. N., Crandall, K. S., Sivo, S. A., \& Reifsteck, J. B. (1999). Relevance of the "BEVI" for research in developmental psychopathology. Poster session presented at the meeting of the American Psychological Association, Boston, MA.

Iyer, C. (2013). Assessing and engaging beliefs and values in a learning community of U.S. and international students: Implications and applications from the Forum BEVI Project. (Unpublished doctoral dissertation). James Madison University, Harrisonburg, VA.

Kehl, K., \& Morris, J. (2007). Differences in global-mindedness between short-term and semester-long study abroad participants at selected private universities. Frontiers: The Interdisciplinary Journal of Study Abroad, XV, 67-80.

Kelley, C. \& Meyers, J. (1995). The Cross-Cultural Adaptability Inventory. Minneapolis, MN: National Computer Systems.

Kline, R. B. (2010) Principles and practice of structural equation modeling (3rd ed.). New York: Guilford Press

Knight, J. (2006). Internationalization of higher education: New directions, new challenges. Global Survey Report. Paris, France: International Association of Universities.

Knight, J. (2011). Five myths about internationalization. International Higher Education, 62, 14-15.

Kohls, L. R. (2001). Survival kit for overseas living (4th edition). Yarmouth, ME: Intercultural Press.

Koskinen, L. \& Tossavainen, K. (2003). Relationships with undergraduate nursing exchange students: A tutor perspective. Journal of Advanced Nursing, 41(5), 499-508.

Kuh, G. D. (2008). High-impact educational practices: What they are, who has access to them, and why they 
matter. Retrieved from https://www.aacu.org/leap/hip.cfm.

Lerner, J. (2003). Learning disabilities: Theories, diagnosis, and teaching practices. Boston, MA: Houghton Mifflin Company.

Lewin, K. (1951). Field theory in social science: Selected theoretical papers. D. Cartwright (Ed.). New York, NY: Harper Brothers Publishers.

Little, D. (2002). The European language portfolio: Structure, origins, implementation and challenges. Language Teaching, 35(3), 182-189.

Manaloa, E., Kusumib, T., Koyasub, M., Michitac, Y., \& Waseda, Y. (2013). To what extent do culturerelated factors influence university students' critical thinking use? Thinking Skills and Creativity 10, 121132.

Marmolejo, F. (2011). The future of higher-education internationalization. Retrieved from http://chronicle.com/blogs/worldwise/defining-internationalization/28615.

Moghaddam, F. M., Harre, R., \& Lee, N. (2007). Global conflict resolution through positioning analysis. New York, NY: Springer Publishing.

NAFSA. Accessing best practices in international education (2008). Retrieved from http://www.nafsa.org/resourcelibrary/default.aspx?id=18067.

Netherlands' Organization for International Cooperation in Higher Education. Mapping Internationalization (MINT) Project (2012). Retrieved from https://www.nuffic.nl/en/expertise/quality-assurance-andinternationalisation/mapping-internationalisation-mint/background-information.

O'Meara, P., Mehlinger, H. D., \& Newman, R. M. (2001). Changing perspectives on international education. Bloomington, IN: Indiana University Press.

Patel, R. (2008). Environmental beliefs, values, and worldviews: Etiology, maintenance, and transformation (Doctoral dissertation). James Madison University, Harrisonburg, VA.

Patel, R., Shealy, C.N., \& De Michele, P. (2007, August). Environmental beliefs and values: Etiology, maintenance, and transformation. Poster session presented at the annual meeting of the American Psychological Association, San Francisco, CA.

Paul, R. \& Elder, L. (2006). The miniature guide to critical thinking: Concepts and tools. Retrieved from http://www.criticalthinking.org/files/Concepts Tools.pdf.

Poole, D., \& Davis, T. (2006). Concept mapping to measure outcomes in study abroad programs. Social Work Education, 25(1), 66-77.

Pysarchik, D. T., Shealy, C. N., Whalen, B. (2007a, February). Antecedents to outcomes-based international learning: A new psychometric assessment approach. Paper presented at the annual meeting of the Association of International Education Administrators, Washington, DC.

Pysarchik, D. T., Shealy, C. N., Whalen, B. (2007b, April). Antecedents to outcomes-based international learning: A new psychometric assessment approach. Symposium presented at the Undergraduate Assessment Symposium, North Carolina State University, Cary, NC.

Ramirez, G. B. (2013). Learning abroad or just going abroad? International education in opposite sides of the 
border. The Qualitative Report, (18)62, 1-11.

Reisweber, J. R. (2008). Beliefs, values, and the development of intercultural awareness (Doctoral dissertation). James Madison University, Harrisonburg, VA.

Rexeisen, R. J. (2012). Study abroad and the boomerang effect: The end is only the beginning. Frontiers: The Interdisciplinary Journal of Study Abroad, XXII, 166-181.

Rexeisen, R. J., Anderson, P. H., Lawton, L., Hubbard, A. C. (2008). Study abroad and intercultural development: A longitudinal study. Frontiers: The Interdisciplinary Journal of Study Abroad, XVII, 1-20.

Robinson, J. P., Shaver, P. R., \& Wrightsman, L. S. (Eds). (1991). Measures of personality and social psychological attitudes. San Diego, CA: Academic Press.

Robinson, J. P., Shaver, P. R., Wrightsman, L. S. (Eds). (1999). Measures of political attitudes. San Diego, CA: Academic Press.

Salisbury, M. H. (2011). The effect of study abroad on intercultural competence among undergraduate college students. (Unpublished doctoral dissertation). University of Iowa, Iowa City, IA. Retrieved from http://ir.uiowa.edu/etd/1073/.

Sampson, D. L. \& Smith, H. P. (1957). A scale to measure world-minded attitudes. The Journal of Social Psychology, 45(1), 99-106.

Scarino, A. (2010). Assessing intercultural capability in learning languages: A renewed understanding of language, culture, learning, and the nature of assessment. The Modern Language Journal, 94(2), 324329.

Schmid, J. \& Leiman, J. N. (1957). The development of hierarchical factor solutions. Psychometrika, 22, 5361.

Scriven, M. \& Paul, R. (1987). Defining critical thinking. Retrieved from https://www.criticalthinking.org/pages/defining-critical-thinking/766.

Shaftel, J., \& Shaftel, T. L. (2010). Measuring intercultural attitudes of undergraduates who study abroad. International Psychology Bulletin, 14(1), 12-15.

Shealy, C. N. (2000a, July). The Beliefs, Events, and Values Inventory (BEVI): Implications for cross-cultural research and practice. Paper presented at the meeting of the International Congress of Psychology, Stockholm, Sweden.

Shealy, C. N. (2000b, July). The Beliefs, Events, and Values Inventory (BEVI): Implications for research and theory in social psychology. Paper presented at the meeting of the International Congress of Psychology, Stockholm, Sweden.

Shealy, C. N. (2004). A model and method for "making" a C-I psychologist: Equilintegration (EI) theory and the Beliefs, Events, and Values Inventory (BEVI). [Special Series]. Journal of Clinical Psychology, 60(10), 1065-1090.

Shealy, C. N. (2005). Justifying the justification hypothesis: Scientific-humanism, Equilintegration (EI) theory, and the Beliefs, Events, and Values Inventory (BEVI). [Special Series]. Journal of Clinical Psychology, 61(1), 81-106. 
Shealy, C. N. (2006). The Beliefs, Events, and Values Inventory (BEVI): Overview, implications, and guidelines. Test manual. Harrisonburg, VA: Author.

Shealy, C. N. (2012) The Forum BEVI Project: Results, applications, and alignments with the Forum Standards of Good Practice. Paper presented at the 8th Annual Conference of the Forum on Education Abroad, Denver, CO.

Shealy, C. N. (2014). Assessing global learning: Implications from the Forum BEVI Project. Workshop presented at the annual meeting of the Association of International Education Administrators, Washington, DC.

Shealy, C. N. (Ed.) (in press). Making sense of beliefs and values. New York, NY: Springer Publishing.

Shealy, C. N., Bhuyan, D., \& Sternberger, L. G. (2012). Cultivating the capacity to care in children and youth: Implications from EI theory, EI self, and BEVI. In Usha S. Nayar (Ed.), Child and adolescent mental health (pp. 240-255). Thousand Oaks, CA: SAGE Publications.

Siaya, L., \& Hayward, F. M. (2003). Mapping internationalization on U.S. campuses. Washington DC: American Council on Education.

Stebleton, M. J., Soria, K. M., \& Cherney, B. T. (2013). The high impact of education abroad: College students' engagement in international experiences and the development of intercultural competencies. Frontiers: The Interdisciplinary Journal of Study Abroad, XXII, 1-24.

Sternberger, L., La Brack, B., \& Whalen, B. (2007). How to begin: Key decision points in the assessment process. In Bolen, M. (Ed.), A guide to assessment for international education. (pp. 71-87). Carlisle, PA: Forum on Education Abroad.

Sternberger, L., Pysarchik, D. T., Yun, Z. S., \& Deardorff, D. K. (2009). Designing a model for international learning assessment. Diversity \& Democracy, 12(1), 7-9.

Sutton, R. C. \& Rubin, D. L. (2004). The GLOSSARI Project: Initial findings from a system-wide research initiative on study abroad learning outcomes. Frontiers: The Interdisciplinary Journal of Study Abroad, X, 65-82.

Tabit, M. B., Cozen, J., Dyjak, K., Pendleton, C., Shealy, C. N., Sternberger, L. G., Staton, R. (2011, August). Sociocultural impact of denial: Empirical evidence from the Forum BEVI Project. Poster presented at the annual meeting of the American Psychological Association.

Tabit, M., Legault, L., Ma, W., \& Wan, P. (in press). In search of best practices for multicultural education: Empirical evidence from the Forum BEVI Project. In C.N. Shealy (Ed.), Making sense of beliefs and values. New York, NY: Springer Publishing.

Tarrant, M. A., Rubin, D. L, \& Stoner, L. (2013). The added value of study abroad: Fostering a global citizenry. Journal of Studies in International Education $X X(\mathrm{X}), 1-21$.

Teddlie, C., \& Tashakkori, A. (2009). Foundations of mixed methods research: Integrating quantitative and qualitative approaches in the social and behavioral sciences. Thousand Oaks, CA: SAGE Publications, Inc.

Vande Berg, M., Connor-Linton, J., \& Paige, M. R. (2009). The Georgetown Consortium Project: Interventions for student learning abroad. Frontiers: The Interdisciplinary Journal of Study Abroad, XVIII, 1-75. 
Van der Zee, K. I., \& Van Oudenhoven, J. P. (2000). The Multicultural Personality Questionnaire: A multidimensional instrument of multicultural effectiveness. European Journal of Personality, 14(1), 291209.

Welch, F. C., Pitts, R. E., Tenini, K. J., Kuenlen, M. G., \& Wood, S. G. (2010). Significant issues in defining and assessing teacher dispositions. Teacher Educator, 45(3), 179-201.

Whalen, B. J. (2011). Introduction. Using the Standards of Good Practice to assess and improve education abroad programs. In The Standards of Good Practice for education abroad. (4 ${ }^{\text {th }}$ ed.) (pp. 3-5). Carlisle, PA: Forum on Education Abroad.

Wild, D., Grove, A., Martin, M., Eremenco, S., McElroy, S., Verjee-Lorenz, A., \& Erikson, P. (2005). Principles of good practice for the translation and cultural adaptation process for patient-reported outcomes (PRO) measures: Report of the ISPOR Task Force for Translation and Cultural Adaptation. Value in Health, 8(2), 94-104.

World Values Survey. Retrieved from http://www.worldvaluessurvey.org/.

Yanchar, S., Slife, B., \& Warne, R. (2008). Critical thinking as disciplinary practice. Review of General Psychology, 12(3), 265-281.

Zimmermann, J., \& Neyer, F.J. (2013, September). Do we become a different person when hitting the road? Personality development of sojourners. Journal of Personality and Social Psychology, 105 (3), 515-530. 